\title{
WestVirginiaUniversity
}

THE RESEARCH REPOSITORY @ WVU

Graduate Theses, Dissertations, and Problem Reports

2017

\section{Radio Transients and their Environments}

\author{
Kaustubh Rajwade
}

Follow this and additional works at: https://researchrepository.wvu.edu/etd

\section{Recommended Citation}

Rajwade, Kaustubh, "Radio Transients and their Environments" (2017). Graduate Theses, Dissertations, and Problem Reports. 6469.

https://researchrepository.wvu.edu/etd/6469

This Dissertation is protected by copyright and/or related rights. It has been brought to you by the The Research Repository @ WVU with permission from the rights-holder(s). You are free to use this Dissertation in any way that is permitted by the copyright and related rights legislation that applies to your use. For other uses you must obtain permission from the rights-holder(s) directly, unless additional rights are indicated by a Creative Commons license in the record and/ or on the work itself. This Dissertation has been accepted for inclusion in WVU Graduate Theses, Dissertations, and Problem Reports collection by an authorized administrator of The Research Repository @ WVU.

For more information, please contact researchrepository@mail.wvu.edu. 


\author{
Radio Transients and their Environments \\ Kaustubh Rajwade \\ Dissertation submitted \\ to the Eberly College of Arts and Sciences \\ at West Virginia University
}

in partial fulfillment of the requirements for the degree of

Doctor of Philosophy in Physics

Duncan Lorimer, Ph.D., Chair

Maura McLaughlin, Ph.D.

Loren Anderson, Ph.D.

Aldo Romero, Ph.D.

Richard Prestage, Ph.D.

Department of Physics and Astronomy

Morgantown, West Virginia

2017

Keywords: Neutron Stars, Pulsars, Fast radio bursts, Scattering, Free-free

Absorption, Radiative transfer

Copyright 2017 Kaustubh Rajwade 


\author{
Abstract \\ Radio Transients and their Environments \\ Kaustubh Rajwade
}

The interstellar medium is the principal ingredient for star formation and hence, it is necessary to study the properties of the interstellar medium. Radio sources in our Galaxy and beyond can be used as a probe of the intervening medium. In this dissertation, I present an attempt to use radio transients like pulsars and fast radio bursts and their interactions with the environment around them to study interstellar medium. We show that radio emission from pulsars is absorbed by dense ionized gas in their surroundings, causing a turnover in their flux density spectrum that can be used to reveal information about the absorbing medium. We carried out a multi-wavelength observation campaign of PSR B0611+22. The pulsar shows peculiar emission variability that is broadband in nature. Moreover, we show that the flux density spectrum of B0611+22 is unusual which can be attributed to the environment it lies in. We also present predictions of fast radio burst detections from upcoming low frequency surveys. We show that future surveys with the Canadian Hydrogen Intensity Mapping Experiment (CHIME) will be able to detect $\sim 1$ radio burst per hour even if the radio burst undergoes significant absorption and scattering. Finally, we present our results of pulsar population synthesis to understand the pulsar population in the Galactic Centre (GC) and place conservative upper limits on the GC pulsar population. We obtain an upper limit of 52 CPs and 10,000 MSPs in the GC. The dense, ionized environment of the GC gives us the opportunity to 
predict the probability of detection by considering scattering and absorption as the principle sources of flux mitigation. Our results suggest that the optimal frequency range for a pulsar survey in the $\mathrm{GC}$ is $9-14 \mathrm{GHz}$. A larger sample of absorbed spectrum pulsars and fast radio bursts will be beneficial not only for the study of emission processes but also for discerning the properties of the material permeating through space. 


\section{Acknowledgments}

I would not have completed this dissertation had it not been for the support and guidance of a number of people, whom I would like to thank. First of all, I would like to thank my advisor, Duncan Lorimer, for being a great mentor to me throughout my graduate school. He was very patient with me and always stimulated innovative discussions which led to this piece of work. I would like to thank Ramesh Karappusamy from Max Planck Institute for Radio Astronomy for giving me the opportunity to work with the instrumentation group which proved beneficial for my overall development. I would like to thank my committee member, Loren Anderson, for guidance with problems related to the interstellar medium. His class on the subject was an inspiration to work in this field. I would like to thank my other committee members - Maura McLaughlin, Aldo Romero and Richard Prestage for their advice during the course of this work and comments on the thesis.

A thanks needs to be extended to my office mates over the past few years Nickolas Pingel and Josh Mascoop - for always being there for much needed motivation and break from work. Nick was especially helpful for discussion research problems whcih could not have been solved without his assistance. I would also like to thank other members of the Astronomy group - Pete Gentile, Lucas Hunt, Michael Lam, Will Armentrout, Matteo Luisi for support throughout my Ph.D. A special thanks to Nate for helping solve all software related problems and taking time out from his busy schedule to work on the roof-top radio telescope and Jayanth for assistance with learning $\mathrm{C}$ during the initial stages of my Ph.D. I would like to thank Akshaya for interesting discussions on fast radio bursts and Harsha for great 
advice before my defense. The department office staff, especially Sherry Puskar and Viola Bryant are appreciated for their assistance with paperwork which cropped up every now and then.

Among the people that I have collaborated with over the last 4 years, a lot of them deserve a special thank you. I would like to thank my collaborators, Aris Karastergiou and Maciej Serylak for help related to the PSR B0611+22 paper. Maciej was crucial in providing calibrated data for B0611+22 from the LOFAR station. My time in Bonn could not have been so productive had it not been for the people that I interacted with in two summers that I spent there. I would like to thank Amit, Alex, Pablo, Joey, Eleni, Nicholas and Jason for making me feel at home in a new country. I would like to thank Yashwant Gupta of NCRA, for providing me my first opportunity to work in radio astronomy.

I cannot finish without thanking all my friends in Morgantown. Without their constant encouragement, I could not have finished my PhD. A special thanks goes to Ted Lane - for showing me the wonders of procrastination. I would also like to thank Matt, Tommy, Pedram, Gary for always providing me with good company and interesting conversations over a drink and the necessary motivation for a game of soccer or basketball on the weekends. I thank Megan and Arturo for being great friends and roommates. Megan's delicious fried catfish and Arturo's silly humour always helped raise my spirits. Finally, I would like to thank my wife, Rohini Joshi, for being a source of inspiration over the last four years. I also would like to thank my parents, who always fostered my liking for Astronomy since childhood. 


\section{Table of Contents}

List of Tables $\quad$ ix

List of Figures $\quad$ X

List of Abbreviations and Symbols $\quad$ xii

1 Introduction 1

1.1 The Radio Universe . . . . . . . . . . . . . . . . . . . . . . . . 1

1.2 Interstellar Medium . . . . . . . . . . . . . . . . . . . 3

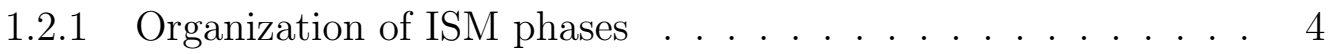

1.2.2 Interstellar Scattering . . . . . . . . . . . . . . 5

1.2.3 Free-free absorption . . . . . . . . . . . . . . . . . 9

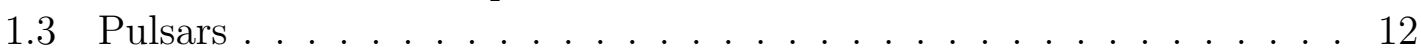

1.3.1 A brief history of the discovery of pulsars . . . . . . . . . . 13

1.4 Properties . . . . . . . . . . . . . . . . . 16

1.4.1 Empirical Model . . . . . . . . . . . . . . . 16

1.4.2 Rotational kinetic energy . . . . . . . . . . . . . . . 19

1.4.3 Magnetic field strength . . . . . . . . . . . . . . 21

1.4.4 Age estimate .................... . . 22

1.4.5 $P-\dot{P}$ diagram of pulsars . . . . . . . . . . . . . . . 23

1.4.6 Dispersion measure $(\mathrm{DM})$. . . . . . . . . . . . . . 26

1.4.7 Spectral Index . . . . . . . . . . . . . . . . . . . . . . . . . . . . . . . . . . . 28

1.5 The pulsar magnetosphere . . . . . . . . . . . . . . . . . . . . 29

1.5.1 Pulsar radio emission . . . . . . . . . . . . . . . . 33

1.5.2 High-energy emission . . . . . . . . . . . . . . . . 37

1.6 Emission geometry and characteristics . . . . . . . . . . . . . . . . . . . . . . . . . .

1.7 Galactic pulsar population . . . . . . . . . . . . . . . . 41

1.7.1 Pulsars in the Galactic Center . . . . . . . . . . . . . . . 43

1.8 Fast Radio Bursts . . . . . . . . . . . . . . . . . . . . . 44

1.8.1 History . . . . . . . . . . . . . . . . . . . . . 45

1.8.2 Properties and emission models . . . . . . . . . . . . . . . . 47

1.8.2.1 Soft Gamma Ray Repeaters . . . . . . . . . . . . . 48

1.8.2.2 Super-giant pulses from neutron stars . . . . . . . . 49

1.8.2.3 Blitzar . . . . . . . . . . . . . . 51

1.8.2.4 Dispersion measures . . . . . . . . . . . . 53

1.8.2.5 Scattering and absorption ........... . . 54

1.8.2.6 Current Results . . . . . . . . . . . . . . . . . . 55

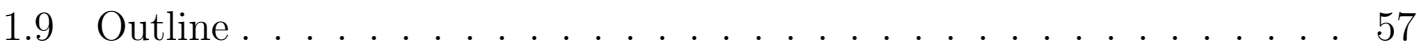


2 On gigahertz spectral turnover in pulsars 58

2.1 Introduction . . . . . . . . . . . . . . . . . . 58

2.2 Model .......................... 61

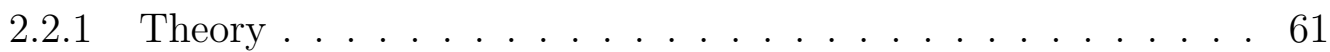

2.2.2 Application .................... 62

2.3 Discussion . . . . . . . . . . . . . . . . . . . . . 68

3 Simultaneous radio and X-ray observations of PSR B0611+22 73

3.1 Introduction . . . . . . . . . . . . . . . . . . . 73

3.2 Observations . . . . . . . . . . . . . . 76

3.3 Results . . . . . . . . . . . . . . . . . . . . . 79

3.3.1 Broadband bursting . . . . . . . . . . . . . . 79

3.3.2 Spectral turnover . . . . . . . . . . . . . . . . 84

3.3.3 Broadband flux density modulation . . . . . . . . . . . . 89

3.3.4 X-ray flux upper limits . . . . . . . . . . . . . . . . . . 90

3.4 Discussion . . . . . . . . . . . . . . . . . . . . . . . . . . . . . 91

3.4.1 Quasi-stable magnetosphere? .............. 91

3.4.2 Flux Density Spectrum . . . . . . . . . . . . . . . 93

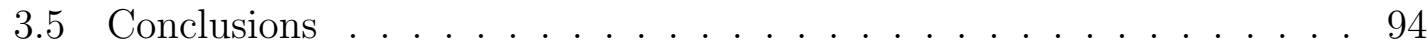

4 Detecting fast radio bursts at decametric wavelengths 96

4.1 Introduction . . . . . . . . . . . . . . . . . . . 96

4.2 Methods . . . . . . . . . . . . . . . . . . 97

4.2.1 Flux-redshift relationship and baseline model . . . . . . . . . 98

4.2.2 FRB survey sensitivity model . . . . . . . . . . . . . . . 101

4.3 Models for flux mitigation . . . . . . . . . . . . . . . . . 103

4.3.1 Models including free-free absorption . . . . . . . . . . . . . 104

4.3.2 Models including multi-path scattering . . . . . . . . . . 107

4.4 Results . . . . . . . . . . . . . . . . . . . 108

4.4.1 Spectral index constraints . . . . . . . . . . . . 108

4.4.2 FRB rate predictions for future surveys . . . . . . . . . . . 110

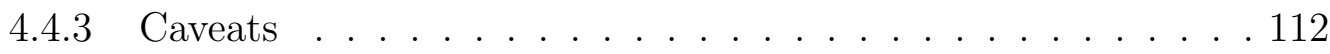

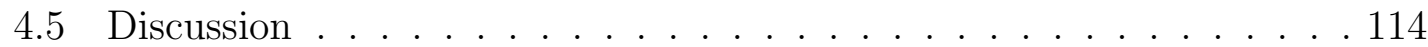

5 Detecting pulsars in the Galactic center 119

5.1 Introduction . . . . . . . . . . . . . . . . . . . . . 119

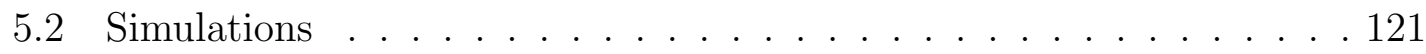

5.2 .1 Model . . . . . . . . . . . . . . . 129

5.2.1.1 Free-free absorption . . . . . . . . . . . . . 129

5.2.1.2 Scattering .................... 131

5.2.2 Probability of detection . . . . . . . . . . . . . . 134

5.2.3 Results........................ 137

5.3 Discussion . . . . . . . . . . . . . . . . . . . . . . . . . 139

5.4 Conclusions . . . . . . . . . . . . . . . . . . . . . . 142 
6 Conclusions 144

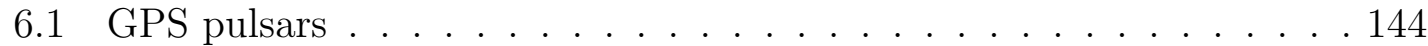

6.2 Simultaneous radio and X-ray observations of PSR B0611+22 . . . 145

6.3 Detecting FRBs at decametric wavelengths . . . . . . . . . . . . 146

6.4 GC pulsar population . . . . . . . . . . . . . . . . . 146

$\begin{array}{ll}\text { A Derivation of optical depth } & 148\end{array}$

B Calculation of scattering efficiency 154 


\section{List of Tables}

2.1 Best-fit parameters for absorbed power-law fit . . . . . . . . . . . 66

2.2 Physical parameters of potential absorbers . . . . . . . . . . . . 67

3.1 Observation details . . . . . . . . . . . . . . . . . . 78

3.2 Flux density of PSR B0611+22 . . . . . . . . . . . . 87

4.1 Survey parameters . . . . . . . . . . . . . . . . 103

4.2 Spectral index constraints on FRBs . . . . . . . . . . . . . 106

5.1 Parameters used for simulation . . . . . . . . . . . . . . . . 124

5.2 Parameters of previous surveys . . . . . . . . . . . . . . 126

5.3 Upper limits on CP and MSP population . . . . . . . . . . . . . 128

5.4 Probability of detection with future GBT surveys . . . . . . . . . 136 


\section{List of Figures}

1.1 Phases of the ISM . . . . . . . . . . . . . . . . . . 3

1.2 Cartoon model for scattering . . . . . . . . . . . . . . . . 6

1.3 Scattered profile of a pulsar . . . . . . . . . . . . . . . 8

1.4 Cartoon model of optical depth . . . . . . . . . . . . . . . 10

1.5 Spectrum for optical depth . . . . . . . . . . . . . . . . . . 12

1.6 Discovery plot of the first pulsar CP $1919 \ldots \ldots \ldots \ldots$. . . . . . 14

1.7 Schematic view of a pulsar magnetosphere . . . . . . . . . 18

1.8 Integrated pulse profiles of pulsars . . . . . . . . . . . . . 20

$1.9 P-\dot{P}$ diagram of pulsars $\ldots \ldots \ldots \ldots \ldots . \ldots \ldots$

1.10 Frequency dependent radio signal of PSR J1832 + $0029 \ldots \ldots$. . . 27

1.11 Pulsar Spectra . . . . . . . . . . . . . . . . . . . 30

1.12 Pulsar magnetosphere . . . . . . . . . . . . . . . . . . 32

1.13 Pair cascade in the pulsar magnetosphere . . . . . . . . . 36

1.14 Core and conal beam structure and patchy beam . . . . . . . . . 39

1.15 Single pulses of PSR B0826-34 . . . . . . . . . . . 40

1.16 Galactic distribution of the known pulsar population . . . . . . . 42

1.17 Detection plot of the Lorimer burst . . . . . . . . . . . . . 46

1.18 Plot showing FRB widths and DMs . . . . . . . . . 54

2.1 Best fit absorbed power-law and standard power-law for PSR B0329+54 66

2.2 Spectral fit of absorbed power-law for our sample of GPS pulsars . . . 67

2.3 Probability density function of turnover frequencies. . . . . . . . . . 71

3.1 Multi-frequency profiles for PSR B0611+22 . . . . . . . . . 80

3.2 Fluctuation spectrum of PSR B0611+22 . . . . . . . . . . 81

3.3 Single bin flux timeseries for B0611+22 . . . . . . . . . . . . 82

3.4 Cross-correlation function of B0611+22 . . . . . . . . . . . . 83

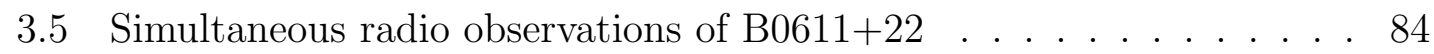

3.6 Average profiles of 2 modes of PSR B0611+22 . . . . . . . . . . 85

3.7 Flux density spectrum of PSR B0611+22 . . . . . . . . . . 88

3.8 Modulation index of PSR B0611+22 $\ldots \ldots \ldots \ldots \ldots$

4.1 Flow chart of the analysis . . . . . . . . . . . . . . . 100

4.2 Figure to illustrate constraint on spectral index . . . . . . . . . 108

4.3 FRB yield predictions . . . . . . . . . . . . . . . 111

4.4 Peak flux versus redshift for CHIME . . . . . . . . . . . . . 112

4.5 Effect of absorption on peak flux . . . . . . . . . . . . . . 115

$5.1 \quad$ K-S Probability of $\gamma \ldots \ldots \ldots \ldots \ldots$

5.2 Synthetic pulsar population luminosity . . . . . . . . . . . . 127

5.3 Scattering efficiency . . . . . . . . . . . . . . . . . . . . . 132

5.4 2-D Histogram showing probability of detection with new backends . 133

5.5 2-D Histogram showing probability of detection with VEGAS backend 138 
A.1 Impact parameter . . . . . . . . . . . . . . . . . . 149

A.2 Fit to Gaunt factor . . . . . . . . . . . . . . . . . . . . 153

B.1 Pulsed fraction as a function of harmonics to be summed . . . . . 156 


\begin{tabular}{ll} 
List of Symbols and Abbreviations \\
$c$ & Speed of light \\
$n_{e}$ & Electron Density \\
$e$ & Charge of electron \\
$m_{e}$ & Mass of electron \\
$G$ & Gravitational constant \\
$k_{b}$ & Boltzmann's constant \\
$\nu$ & Frequency \\
$B_{\nu}$ & Spectral Radiance \\
$\tau$ & Optical Depth \\
M $\odot$ & Solar Mass \\
pc & Parsec \\
$G$ & Gauss \\
$T$ & Tesla \\
J & Joule \\
GBT & Green Bank Telescope \\
AO & Arecibo Observatory \\
WVU & West Virginia University \\
LOFAR & LOw Frequency ARray \\
CHIME & Canadian Hydrogen Intensity Mapping Experiment \\
HIRAX & Hydrogen Intensity Real-time Analysis eXperiment \\
XMM & X-ray Multi-Mirror \\
SKA & Square Kilometer Array \\
ngVLA & new generation Very Large Array \\
EM & Emission Measure \\
SNR & SuperNova Remnant \\
PWN & Pulsar Wind Nebula \\
IGM & InterGalactic Medium \\
ISM & InterStellar Medium \\
CMB & \\
HII & Cosmic Microwave Background Hydrogen \\
\hline & \\
\hline &
\end{tabular}




$\begin{array}{ll}\text { CO } & \text { Carbon Monoxide } \\ \text { HIM } & \text { Hot Ionized Medium } \\ \text { CP } & \text { Cambridge Pulsar } \\ \text { PSR } & \text { Pulsating Source of Radio } \\ \text { LGM } & \text { Little Green Men } \\ \text { LMC } & \text { Large Magellanic Cloud } \\ \text { SMC } & \text { Small Magellanic Cloud } \\ \text { RA } & \text { Right Ascension } \\ \text { EPN } & \text { European Pulsar Network } \\ \text { ATNF } & \text { Australia Telescope National Facility } \\ \text { NCRA } & \text { National Centre for Radio Astrophysics } \\ \text { L } \odot & \text { Solar Luminosity } \\ \text { LTE } & \text { Local Thermodynamic Equilibrium } \\ \text { UV } & \text { Ultra-Violet } \\ \text { AGN } & \text { Active Galactic Nuclei } \\ \rho & \text { charge density } \\ \vec{E} & \text { Electric Field } \\ \vec{B} & \text { Magnetic Field } \\ \dot{E} & \text { Spin-down luminosity } \\ \Omega & \text { Angular velocity } \\ P & \text { Spin period } \\ \dot{P} & \text { Period derivative } \\ I_{\nu} & \text { Spectral intensity } \\ h & \text { Planck's constant } \\ \omega & \text { Angular frequency } \\ e^{-} & \text {Electron } \\ e^{+} & \text {Positron } \\ \gamma & \text { Lorentz factor } \\ & \end{array}$




\section{Chapter 1}

\section{Introduction}

\subsection{The Radio Universe}

Over the past century, astronomers have studied the Universe using the entire electromagnetic spectrum. Radio astronomy is one such branch of astronomy that has changed our perception about the Universe over the last seven decades. It has not only been crucial in studying Hydrogen, the most abundant element in space, but also has been essential to probe formation and evolution of the entire Universe.

Radio Astronomy started in the 1930s, when a young engineer called Karl Guthe Jansky was hired by Bell labs to study the static in trans-atlantic transmission lines. In 1929, Jansky designed and built an antenna that was steerable and could scan the sky in 20 minutes. The hiss that was present in the line was thought to be due to the Sun. After further study, Jansky realized that the hiss repeated after every 23 hours and 56 minutes, which is equal to the sidereal period of the Earth and not 24 hours, which it should have been if it were coming from the Sun. In this way, Jansky was able to conclude that the noise in the transmission line was due to a signal coming from the center of the Milky Way in the constellation of Sagittarius. This discovery revolutionized astronomy and gave birth to the field of radio astronomy.

Since then, a variety of astrophysical sources have been found to emit radio 
waves. One of the most important characteristic of radio astronomy is that astronomers can detect objects that emit non-thermal radiation i.e. radiation that does not depend on the physical temperature of the emitting source. For example, synchrotron radiation from relativistic charged particles spiraling around magnetic fields can be observed by radio telescopes. Similarly, pulsars, which emit coherent radio emission from their magnetic poles are also detected by radio telescopes. Observations at radio wavebands has led to significant advancement of astronomy. These include detection of the cosmic microwave background (CMB), the relic radiation from recombination of ions and electrons in the early Universe, and Active Galactic Nuclei (AGNs) emitting radio waves that are powered by the supermassive black holes. Radio recombination lines, which are transitions of electrons through different states after recombining with the Hydrogen nucleus, are radio emission lines. These lines can be used as a diagnostic for star forming regions that are opaque to optical wavelengths due to extinction by dust. Moreover, Hydrogen, which is the most abundant and the most fundamental element in the Universe, emits at radio wavelengths and hence is the only way to probe neutral hydrogen in the Universe. Similarly, molecular Hydrogen, which is hard to detect from emission lines, can be probed using $\mathrm{CO}$ emission, which is in the radio waveband. All this evidence brings out an important point that Radio Astronomy can be used to study cold objects and Hydrogen in space that are rendered invisible to other wavelengths. This shows that radio astronomy has not only led to major scientific discoveries but also complements studies at other wavelengths. Since radio waves are weak in strength, we need to build large dishes to collect all the radiation that 


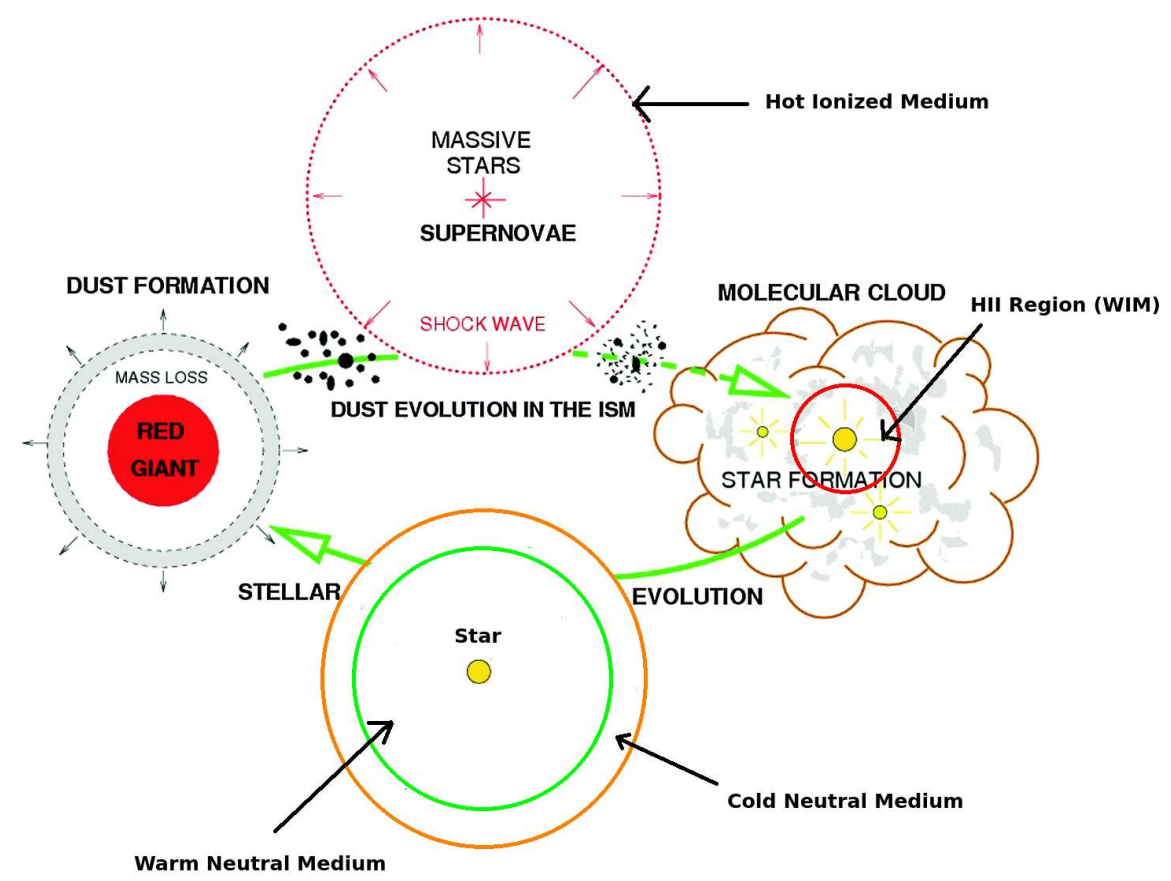

Figure 1.1: Illustration of different phases in the ISM and the interaction between them.

we can to observe these sources. The study of radio sources over the last several decades has shed light on important questions regarding formation and evolution of the Universe, galaxies and the stars within them.

\subsection{Interstellar Medium}

The ISM consists of gas and dust in the Galaxy. It is a vital component of the Galaxy and is responsible for the formation of stars. In the early Universe, most of the baryonic matter was in the interstellar gas that later evolved into galaxies and stars. The ISM has a complex structure and can be found in different phases. 


\subsubsection{Organization of ISM phases}

In a spiral galaxy like the Milky Way, most the dust and gas is situated in the Galactic plane. Though the ISM extends above and below the disk, about $80 \%$ of it lies within a few hundred parsec of the Galactic midplane. The gas is mostly made up of Hydrogen and Helium. The ISM in different regions of the Galaxy have different temperatures and densities which determines the evolution of the Galaxy in that region. The different phases in the ISM are

- Coronal Gas: Coronal gas, also termed as "Hot Ionized Medium", consists mostly of gas that has been shock heated to temperatures of $\sim 10^{5.5} \mathrm{~K}$ from blastwaves from supernova explosions. The gas has very low density $(\sim 0.001$ $\mathrm{cm}^{-3}$ ) but occupies about half of the volume of the Galactic disk.

- Warm Ionized medium (WIM): WIM is found in our Galaxy in the form of regions of ionized Hydrogen where stars are born. This mostly consists of diffuse free electrons with densities ranging from $0.01-0.1 \mathrm{~cm}^{-3}$. The mean temperature of the WIM is $\sim 10^{4} \mathrm{~K}$. The Hydrogen is ionized by ultra-violet photons coming from hot young stars.

- Warm Neutral Medium: Warm Neutral Medium is predominantly neutral Hydrogen gas at temperatures of $\sim 10^{3.7} \mathrm{~K}$. This medium is mostly responsible for the 21-cm emission that we observe everywhere in our Galaxy. The density of gas is $\sim 0.6 \mathrm{~cm}^{-3}$.

- Cold Neutral Medium: Cold Neutral Medium consists of atomic gas at 
temperatures $\sim 10^{2} \mathrm{~K}$ with densities of $\sim 30 \mathrm{~cm}^{-3}$. The gas fills about $1 \%$ of the total Galactic volume.

- Molecular Hydrogen: Molecular Hydrogen is a dominant fraction $(\sim 0.9)$ of the mass of giant molecular cloud complexes in the ISM. This is the main ingredient for star formation in the Galaxy. The densities achieved in these clouds can be as high as $\sim 10^{3} \mathrm{~cm}^{-3}$. These are dark clouds that are traced using $\mathrm{CO}$ emission lines

The different phases of the ISM can also be seen in Figure 1.1. Radio waves traveling from different sources interact with the ISM, the signatures of which are evident in the received signal. Below, I will briefly discuss few of the known phenomena related to this interaction.

\subsubsection{Interstellar Scattering}

As radio waves travel through the ISM, they get deflected due the electron density fluctuations in the intervening medium. Hence, the waves received on Earth are scattered and those signatures are seen in the observed data. Scattering using was first studied by Scheuer (1968) using pulsars (see section 1.3) and since then, there have been multiple detailed treatments of the problem (Rickett, 1977; Bhat et al., 2004; Lewandowski et al., 2015; Krishnakumar et al., 2015).

Consider a radio wave traveling through a screen of cold plasma with an electron density fluctuation $\Delta n_{e}$ as shown in Figure 1.2. Then, the phase shift in the 

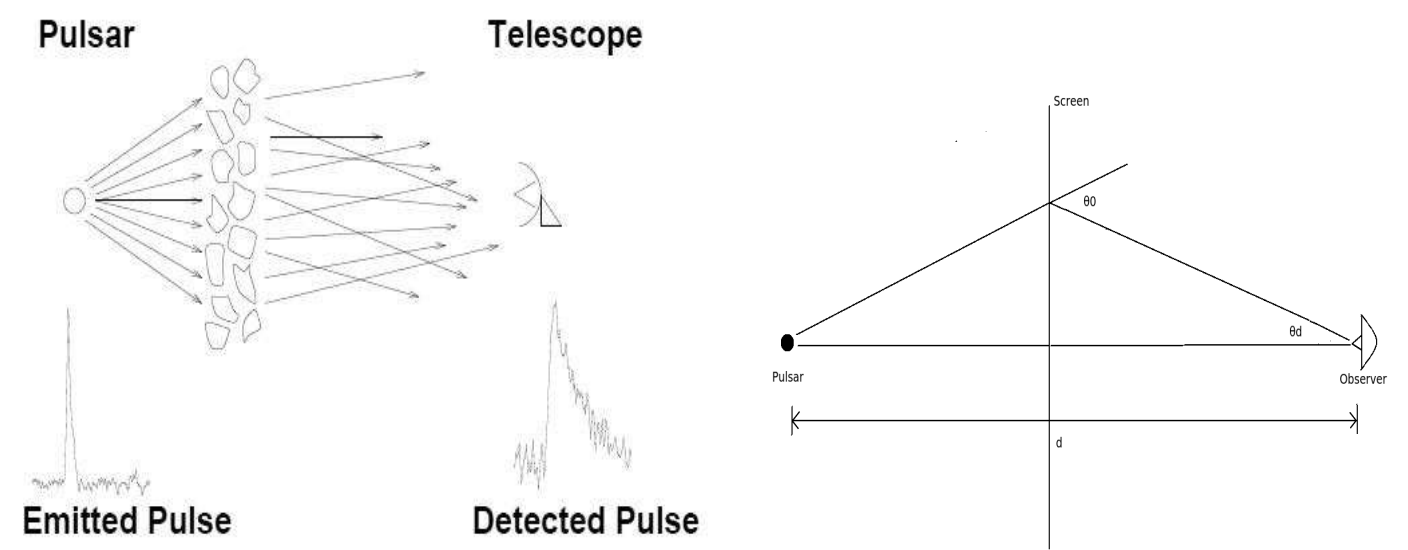

Figure 1.2: Cartoon diagram to explain thin screen scattering. The left panel illustrates the phenomenon of scattering observed in pulsars. The right panel shows one such ray path for the mathematical treatment in the section. We assume that the scattering screen is at exactly at the mid-point between the pulsar and the observer.

wave,

$$
\delta \phi=\Delta k a,
$$

where $a$ is the length scale of electron density fluctuation and $\Delta k$ is the change in magnitude of the wave vector $\vec{k}$. We assume that the plasma is uniform, isotropic and coherent. Then, we can define $\mu$ to be the refractive index of the medium such that,

$$
\mu=\sqrt{1-\left(\frac{f_{p}}{f}\right)^{2}},
$$

where $f$ is the observing frequency and the plasma frequency,

$$
f_{p}=\sqrt{e^{2} n_{e} / \pi m_{e}} .
$$

where $n_{e}, m_{e}$ and $e$ is the density, mass and charge of the electron respectively. 
Plugging in $k=2 \pi \mu \nu / c$, Eq 1.2 and Eq 1.3 in Eq 1.1 we get

$$
\delta \phi=\Delta k a \approx \frac{2 e^{2} a \Delta n_{e}}{c m_{e} f} .
$$

If the wave encounters $d / a$ such fluctuations, the root mean square change in phase,

$$
\Delta \Phi=\sqrt{\frac{d}{a}} \delta \phi=\frac{2 e^{2}}{m_{e} c} \frac{\sqrt{a d} \Delta n_{e}}{f} .
$$

The overall effect of these phase changes is that the observer sees the wave scattered by angle,

$$
\theta_{\mathrm{sca}}=\frac{\Delta \Phi / k}{a} \approx \frac{e^{2}}{\pi m_{e}} \frac{\Delta n_{e}}{\sqrt{a}} \frac{\sqrt{d}}{f^{2}} .
$$

Hence, an observer would see a scattered image of a point source with an angular radius,

$$
\theta_{\mathrm{d}}=\theta_{\mathrm{sca}} / 2 \approx \frac{e^{2}}{2 \pi m_{e}} \frac{\Delta n_{e}}{\sqrt{a}} \frac{\sqrt{d}}{f^{2}} .
$$

In the simplest case, we assume a Gaussian scattering screen hence, the intensity distribution

$$
I(\theta) d \theta \propto \exp \left(-\frac{\theta^{2}}{\theta_{\mathrm{d}}^{2}}\right) 2 \pi \theta d \theta .
$$

Since the scattered wave has to travel a larger distance compared to the wave traveling directly to the observer (see Figure 1.2), using simple geometry and small angle approximation, one can show that it will be delayed from the direct wave by

$$
\Delta t(\theta)=\frac{\theta^{2} d}{c} .
$$




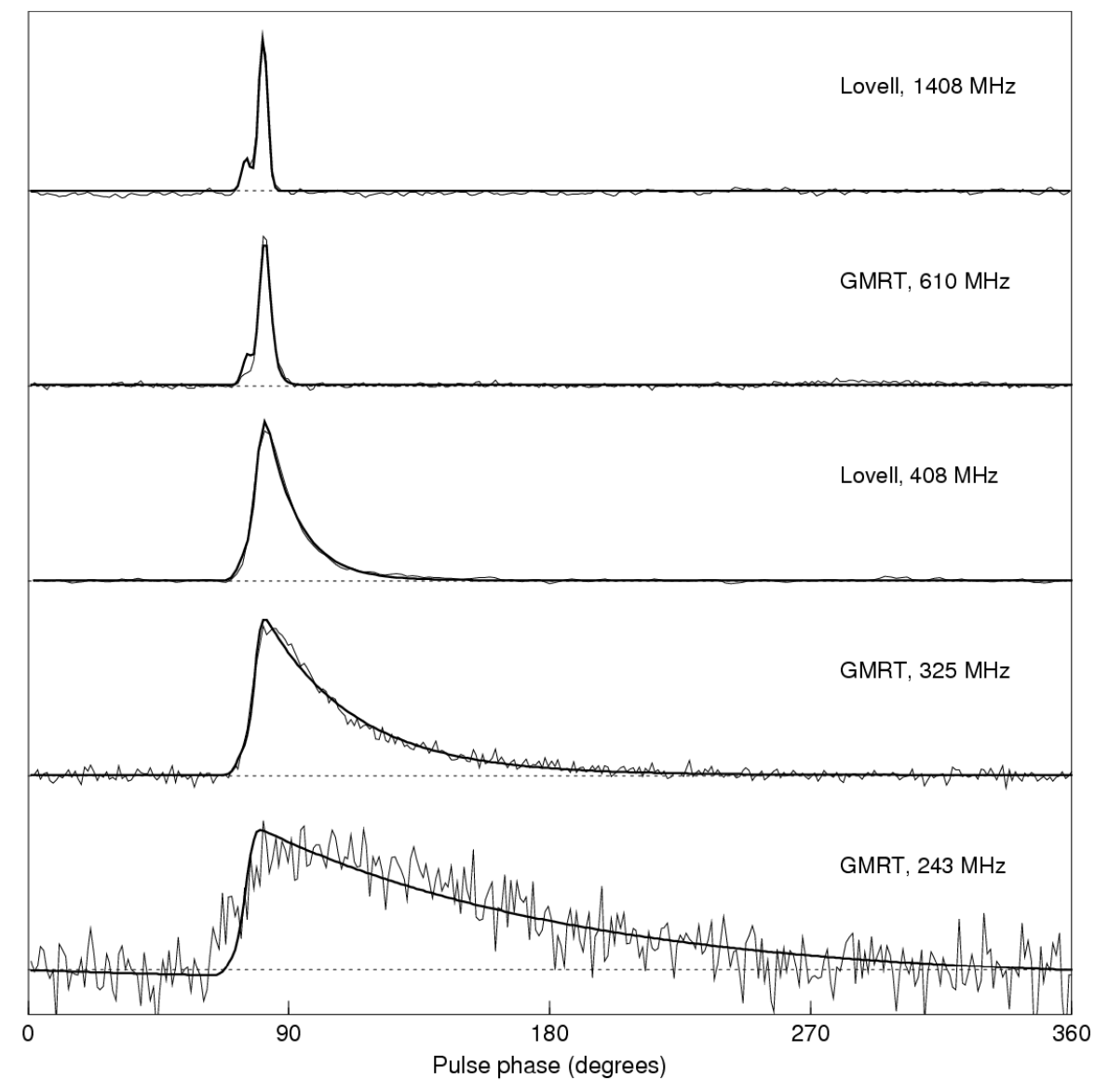

Figure 1.3: Figure showing effect of scattering on the pulsar profile of PSR B183103. One can see the increase in the scattering tail at lower frequencies. Figure taken from Lorimer \& Kramer (2005).

Inserting this result in Eq. 1.9, we see that

$$
I(t) \propto \exp \left(\frac{-c \Delta t}{\theta_{\mathrm{d}}^{2} d}\right)=e^{-\Delta t / \tau_{s}}
$$

where

$$
\tau_{s}=\frac{\theta_{d}^{2} d}{c}=\frac{e^{4}}{4 \pi^{2} m_{e}^{2}} \frac{\Delta n_{e}^{2}}{a} d^{2} f^{-4} .
$$


Thus, a narrow pulse will have a scattering exponential tail with the magnitude of scatter dependent on the frequency of observation. Many pulsars have been observed to have similar exponential tails (see Figure 1.3 for an illustration). Any complicated pulse shape will appear as a convolution of the intrinsic pulse shape and the scattering function. One must note that this mathematical treatment of scattering is a simple case and has been shown to be a reasonable approximation in our Galaxy. Bhat et al. (2004) and Krishnakumar et al. (2015) have performed scattering measurements of a large sample of pulsars to show that scattering time scales with frequency as $\sim-4$ power. Sutton (1971) studied giant pulses from the Crab pulsar to test the thin screen hypothesis since we can approximate the giant pulses as a unit impulse response function. These studies have shown the thin screen model to be a good approximation to describe scattering in our Galaxy. Also, we have assumed a Gaussian scattering screen and that is not always the case. The scattering problem can become complicated for scattering screens with complex and anisotropic density fluctuations (Lewandowski et al., 2015; Geyer \& Karastergiou, 2016).

\subsubsection{Free-free absorption}

In certain regions of the Galaxy, We can approximate the ISM to be in local thermodynamic equilibrium (LTE). In LTE, the energy levels in atoms are populated according to the thermal distribution in equilibrium. This suggests that to keep the temperature constant, there is a balance between emission and absorption 


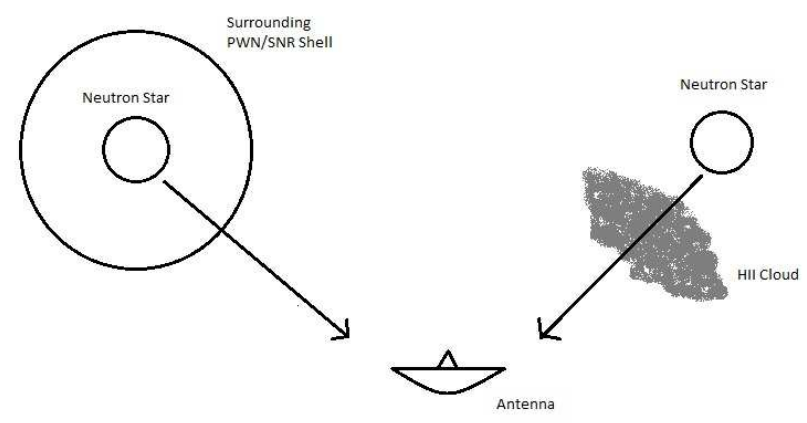

Figure 1.4: Cartoon diagram to show free-free absorption due to dense medium along the line of sight to the pulsar. We can quantify this absorption by defining an optical depth (see text for details).

of radiation. Free-free absorption arises from absorption of incoming radiation by free electrons. According to Larmor's formula, an electron will radiate if it is accelerated by an external force. By similar logic, an electron absorbs radiation if it is decelerated by an external force and is called free-free absorption. Since radiation is being absorbed, one can think of the ISM as being opaque to the radiating source beyond it, if the absorption is significant. Astronomers call this an "optically thick" ISM. On the contrary, regions transparent to distant sources are "optically thin". The amount of absorption is a function of the observing frequency and the temperature and density of the free electrons along the line of sight (see Appendix A). There are regions in our Galaxy with enhanced free electron densities and temperatures (e.g. HII regions, supernova remnants), which can be the source of absorption. The densities and temperatures of such regions favor absorption of radio photons and hence it is an important effect to consider when studying radio sources that are beyond dense, ionized structures within the Galaxy.

Absorption has been observed in spectra of many supernova remnants (Dulk \& Slee, 1975). Radio pulses emitted by fast spinning neutron stars (see section 1.3) 
traveling through dense, ionized regions in space are absorbed by the medium (see Figure 1.4), resulting in gigahertz frequency turnovers that are observed in a number of pulsar spectra (Kijak et al., 2007, 2011). Absorption can be quantified by an optical depth,

$$
\tau=\int_{0}^{l} \kappa d l
$$

where $\kappa$ is the absorption coefficient that is derived in detail in Appendix A, $d l$ is the line element along the line of sight and $l$ is the total distance. Figure 1.5 shows the spectrum of a source in different regimes of optical depth. One can observe a turnover in the spectrum at $\tau \simeq 1$. When $\tau \ll 1$, the intervening medium is optically thin and we can observe emission from the source through the absorbing slab while we can observe emission only from the intervening medium if it is optically thick $(\tau \gg 1)$. Since the total observed intensity is proportional to the balance between absorption and emission, following Rybicki \& Lightman (1979), if $I_{\nu}$ is the intensity received by the telescope (as shown in Figure 1.4) then, assuming LTE,

$$
I_{\nu}\left(\tau_{\nu}\right)=I_{\nu}(0) e^{-\tau_{\nu}}+S_{\nu}\left(1-e^{-\tau_{\nu}}\right)
$$

where $I_{\nu}(0)$ is the intensity emitted from the background and $S_{\nu}$ is the source function that is given by,

$$
S_{\nu}=\frac{j_{\nu}}{\kappa_{\nu}}
$$

where $j_{\nu}$ is the emissivity and $\kappa_{\nu}$ is the absorption coefficient. In LTE, $S_{\nu}$ becomes $B_{\nu}(T)$ which is the blackbody radiation at temperature $T$ from the intervening 


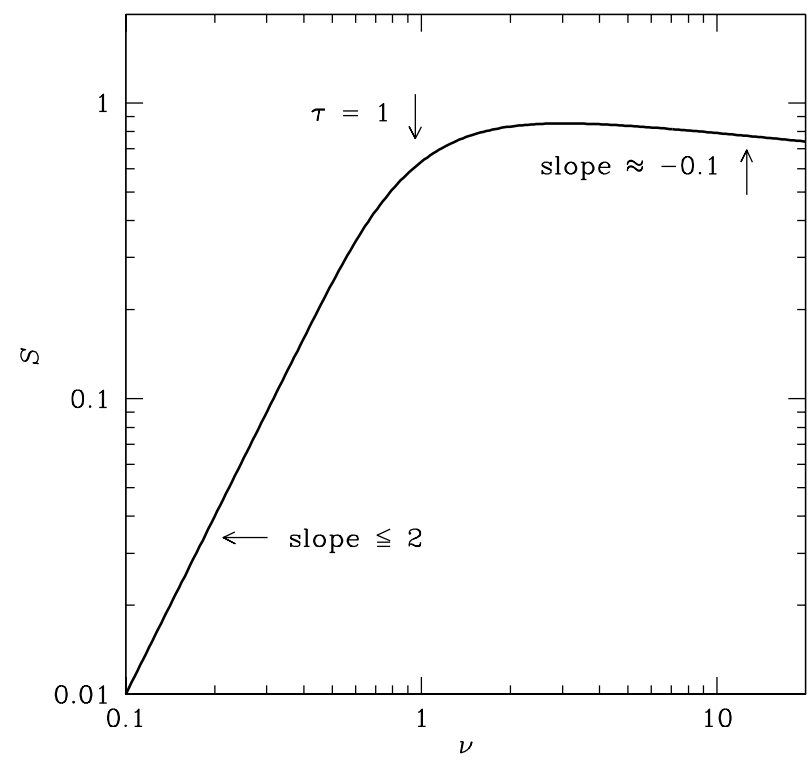

Figure 1.5: Spectrum of free-free emission. One can see a turnover at a frequency where $\tau \approx 1$ due to free-free absorption. Figure taken from Condon \& Ransom (2016).

medium. One can simplify the equation depending on whether the ISM is optically thick or thin.

Scattering and free-free absorption are responsible for modulating the signal received from various radio sources like pulsars and fast radio bursts discussed in later sections. By modeling these signatures, we can use the radio sources as probes of the ISM and learn about the physical characteristics of the ISM in the Galaxy.

\subsection{Pulsars}

Pulsars are rapidly rotating neutron stars that emit coherent radio emission from their magnetic poles and possess extremely high magnetic fields. These are born from supernova explosions of massive stars. Neutron stars are highly compact objects with densities comparable with that of an atomic nucleus. Pulsars have been 
studied across the electromagnetic spectrum (Hermsen et al., 2013; Abdo et al., 2013) and are a key instrument in the detection of low frequency gravitational waves (Arzoumanian et al., 2016).

\subsubsection{A brief history of the discovery of pulsars}

The discovery of pulsars is one of many serendipitous discoveries in science. In 1967, Jocelyn Bell Burnell was a graduate student at University of Cambridge. Along with her advisor, Anthony Hewish, she built a radio telescope to study quasars using interplanetary scintillations. The data were recorded on chart recorders that were hundreds of meters in length. Since interplanetary scintillations are on the order of a few seconds, the sampling rate of the recorder was a fraction of a second, comparable to the rotation period of pulsars. The recorded data included radio signals from space and interference from terrestrial sources. While searching through this immense data set, Bell found a peculiar signal that was highly periodic. When she approached Hewish, he thought it was interference. Soon, it was realized that the signal was always in the data and would start four minutes later than the previous day suggesting that, like Jansky's source, it followed Earth's sidereal period. This confirmed that it was a celestial source. Radio follow-up of the source confirmed its periodic nature and the period was determined to be 1.33 seconds. The source was

named Little Green Man-1 (LGM1) since it was thought that the signals could be from extra-terrestrial life forms.

Soon after this discovery, Bell found a similar source at a different position in 


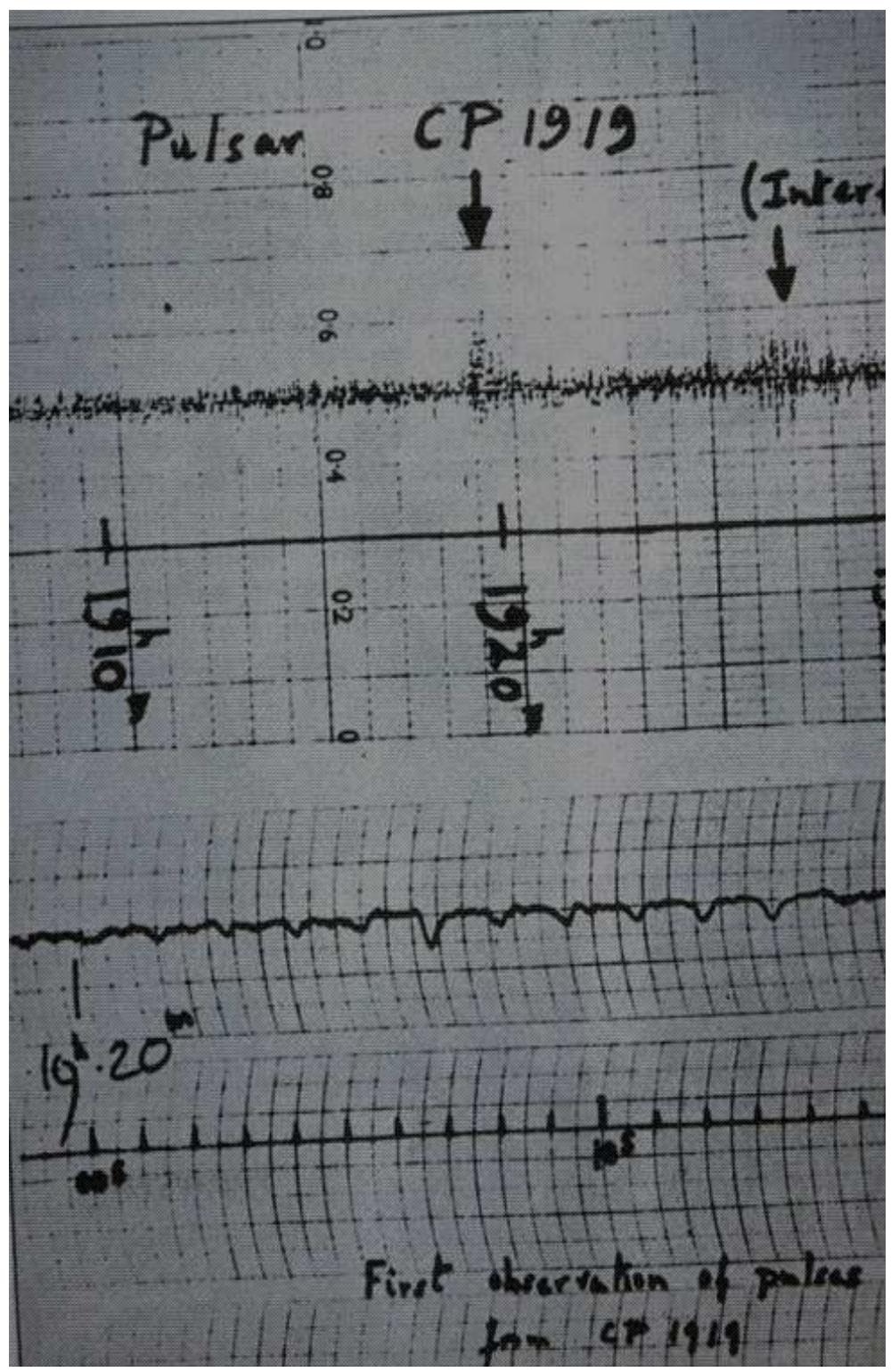

Figure 1.6: Discovery observation of the first pulsar LGM-1, or CP 1919 (now it is known as PSR J1921+2153). The upper image shows the original detection and the lower one shows the high time resolution observation. Figure taken from Lorimer \& Kramer (2005). 
the sky. Follow-up observations further confirmed these two sources and identified two new sources, revealing that LGM-1 was not unique. Therefore, LGM-1 was named as Cambridge Pulsar (CP) 1919 according its right ascension (19h 19m) and other three were named as CP 1133, CP 0834 and CP 0950. After confirming observations and data, they published the discovery of the new astronomical object CP 1919 on February 28, 1968 (Hewish et al., 1968). The second paper came out on April 13 reporting the discovery of the other three sources and further timing results of CP 1919 (Pilkington et al., 1968). A larger sample of pulsars required a more systematic way of naming them uniquely. In order to do that, pulsars are now named according to their position in the sky with right ascension and declination with the first alphabet denoting the epoch; i.e., CP 1919, CP 1133, CP 0834, and CP 0950 are named as PSRs J1921+2153, J1136+1551, J0837+0610, and J0953+0755, respectively where J stands for J2000 epoch.

Since then, we have discovered more than 2500 pulsars in the Galaxy, with at least 30 of them in our satellite galaxies, namely, the Small and Large Magellanic Clouds and $\sim 150$ in globular clusters (Hobbs et al., 2005). These sources are special tools for understanding basic theories in physics. They are also ideal sources for experiments that we cannot conduct in laboratories such as testing General Relativity (GR). 


\subsection{Properties}

It is fair to say that even after 50 years of pulsar research, we do not have a complete understanding of pulsar emission physics. There are a lot of questions about the pulsar magnetosphere, the plasma densities, the structure of the star and the radio and high energy emission that are yet to be answered. For an excellent introduction on our current understanding of pulsars, see Melrose (1995).

A massive star $\left(\sim 8-25 \mathrm{M}_{\odot}\right)$ during the end of its life undergoes a brilliant explosion termed as a core collapse supernova leaving just the neutron-rich core behind, logically called the neutron star (NS hereafter). This highly magnetized $\left(\sim 10^{8} \mathrm{~T}\right)$ compact star has a short spin period $(0.001-1$ second $)$ and almost all of its energy is released by loss of the rotational kinetic energy mainly through the ejection of relativistic particles and the emission of non-thermal electromagnetic radiation. The electromagnetic radiation (from radio to gamma-ray wavelengths) from the NS is observed as pulsations due to the NS spin. This NS is called a pulsar as the beam traverses our line of sight. Neutron stars are very dense objects with typical masses of $\sim 1.4 \mathrm{M}_{\odot}$ within a radius of $\sim 10 \mathrm{~km}$.

\subsubsection{Empirical Model}

In general, the NS magnetosphere can be understood as a magnetic dipole. Figure 1.7 shows a "toy model" of a pulsar magnetosphere, sometimes known as the "lighthouse model". The radio emission of pulsars originates within the inner acceleration gap and is centered around the magnetic axis (Gil \& Melikidze, 2002). 
Thus, the radio emission is low-altitude, extending from the NS surface up to about a few hundred kilometers, and emitted as a narrow beam (Dyks et al., 2004). In general, we assume pulsars are dipolar, having two radio beams, one for each magnetic pole. With a misaligned magnetic axis, we detect pulses from the pulsar when its radio beam crosses our line-of-sight while it is spinning, analogous to a lighthouse beam. On the other hand, the high-energy gamma-ray emission of pulsars originates at higher altitudes within outer acceleration gaps in the outer magnetosphere (see Figure 1.7). Therefore, gamma-ray pulsars have wider fan-like beams, covering a larger fraction of the sky compared to radio beams (Romani \& Yadigaroglu, 1995).

The individual radio pulses of pulsars are very weak signals and can only be seen from brighter sources. Therefore, we can stack a number pulses from a pulsar at the known periods such that the pulsar signal becomes stronger, a process known as "folding", and create a total pulse profile which is known as the "integrated pulse profile". Its vertical axis represents the intensity and the horizontal axis represents a spin rotation in degrees $\left(0^{\circ}-360^{\circ}\right)$, or spin phase. The shape of the pulse profile depends on the viewing geometry, the intrinsic beam structure of the pulsar and scattering, if any, along the line of sight, which is unique for the given source. For instance, if the misalignment between the magnetic and rotational axis of the pulsar is $0^{\circ}$, we do not see pulses as the pulsar will not lose energy via dipolar radiation. On the other hand, if the misalignment is close to $90^{\circ}$ and our line-ofsight is nearly perpendicular to the rotation axis, we observe radio emission from both poles of the pulsar, resulting in a main pulse and an interpulse in the pulse profile with a separation of about $\sim 180^{\circ}$ though interpulses can also arise from a 


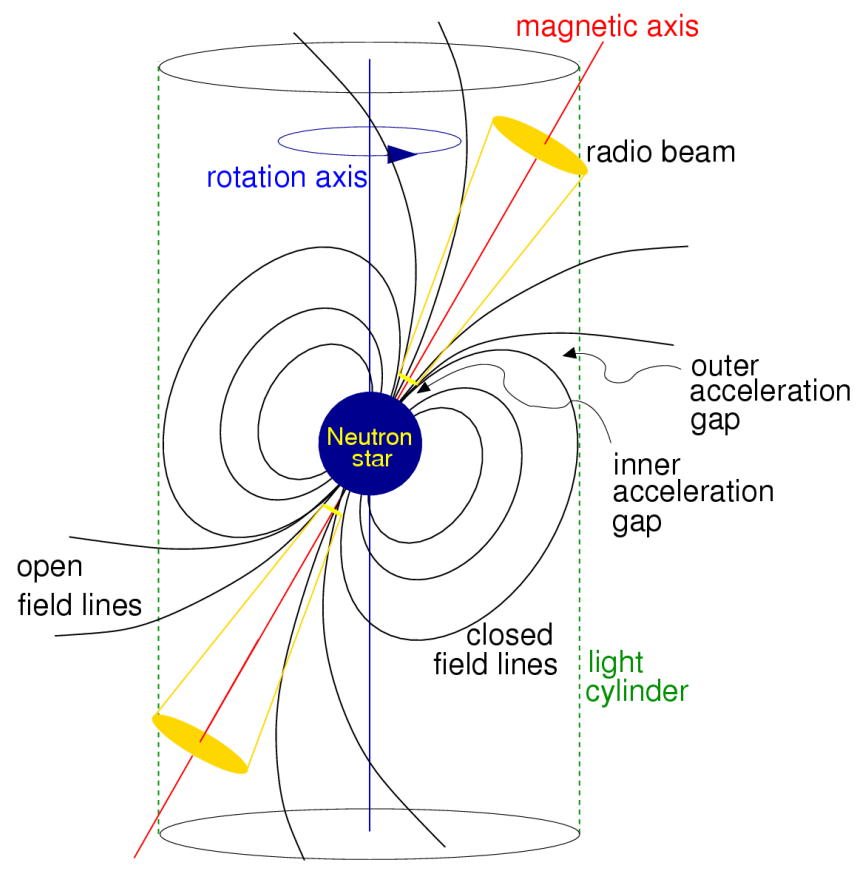

Figure 1.7: Lighthouse model of a rotating pulsar and its magnetosphere (Lorimer \& Kramer, 2005). The magnetic axis is misaligned with the vertical rotation axis. The closed field lines are defined with respect to the light cylinder, which is marked with dotted lines. The light cylinder is an imaginary boundary of the NS magnetosphere where the corotating particles obtain the maximum velocity, the speed of light. The field lines that are outside of this boundary are known as open field lines. The radio beams are located at each pole of the NS centered around the magnetic axis. 
single magnetic pole (Manchester \& Lyne, 1977). For most pulsars that we observe, we see only one pulse since only one magnetic pole traverses our line of sight and the misalignment can vary between $0^{\circ}$ to $90^{\circ}$. Weltevrede \& Johnston (2008) have shown that though pulsars are born with random misalignments of the magnetic pole with the rotation axis, they tend to align themselves on a timescale of $\sim 7 \times 10^{7}$ years which has implications on the detectibility and total population of neutron stars in the Galaxy. In addition to the geometry, the pulse profile shape depends on the radio flux across the beam, which is not uniform and has a complicated structure (for further discussion, see Manchester et al., 2010). Therefore, the observed pulse profile may consist of several components in the main pulse or interpulse (e.g. Rankin, 1983; Gangadhara \& Gupta, 2001). Figure 1.8 shows some examples of different integrated pulse profile shapes.

\subsubsection{Rotational kinetic energy}

Pulsars are powered by loss of their rotational kinetic energy. Therefore, the spin period of a pulsar increases with time. With the rotational energy $E_{\text {rot }}=I \Omega^{2} / 2$, we write the rotational kinetic energy loss as

$$
\dot{E}=\frac{d E_{\mathrm{rot}}}{d t}=\frac{d\left(I \Omega^{2} / 2\right)}{d t}=I \Omega \dot{\Omega}=4 \pi^{2} I \dot{P} P^{-3},
$$

where $\Omega$ is the rotational angular frequency, $P$ is the spin period, and $\dot{P}$ is the derivative of the spin period. This quantity $\dot{E}$ is known as the spin-down luminosity of the pulsar. By assuming $I=10^{38} \mathrm{~kg} \mathrm{~m}^{2}$ (for a NS with mass $=1.4 \mathrm{M}_{\odot}$ and 

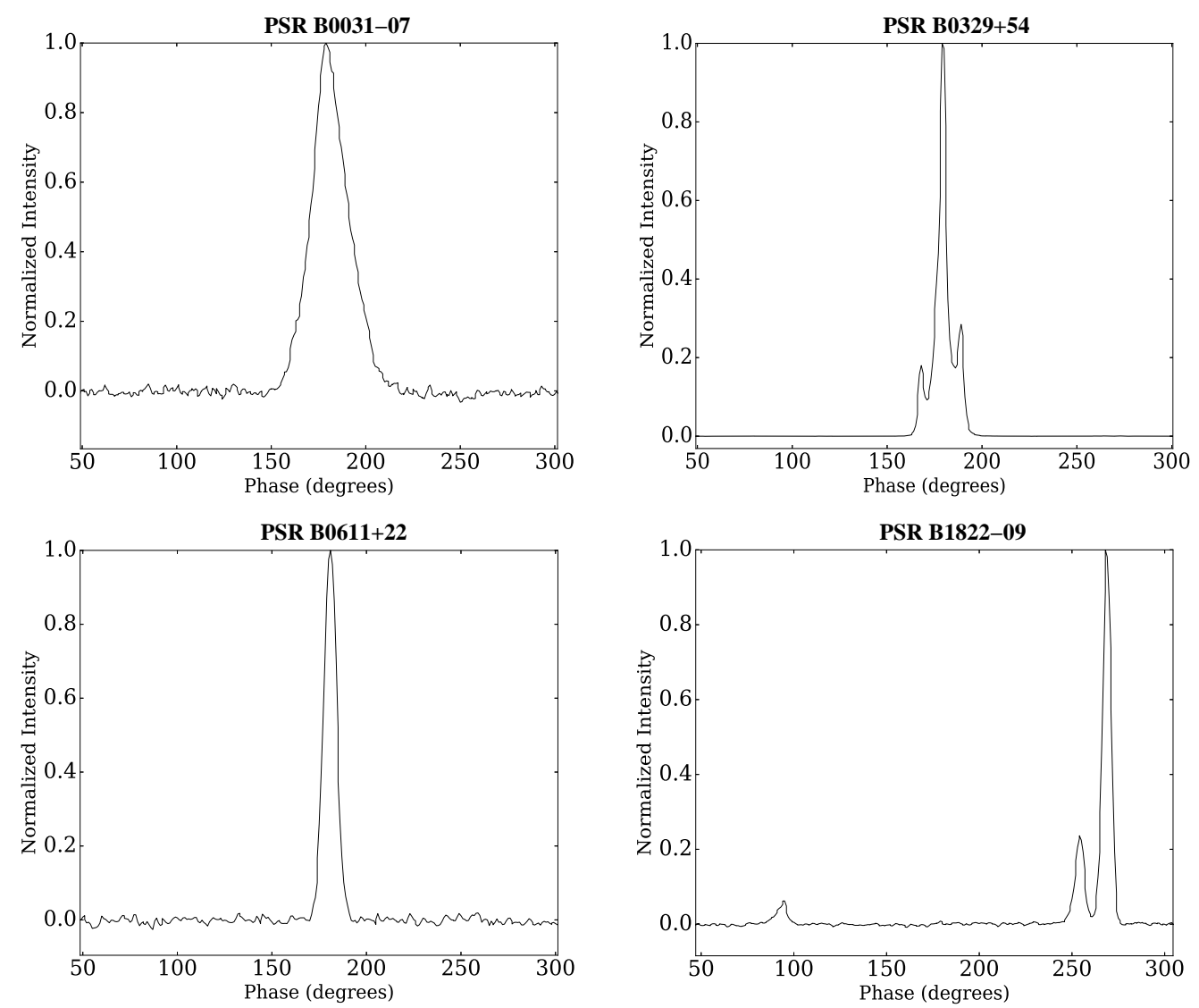

Figure 1.8: Integrated pulse profiles of four pulsars at $1408 \mathrm{MHz}$. The profile of B1822-09 shows the main pulse and the interpulse. Profiles of PSR B0031-07 and PSR B0611+22 show single components while profile of PSR B0329+54 shows multiple components. Data taken from EPN pulsar database (http://www . jb. man . ac.uk/pulsar/Resources/epn/). 
radius $=10 \mathrm{~km}$ ), we can rewrite the equation as

$$
\dot{E}=3.95 \times 10^{24}\left(\frac{\dot{P}}{10^{-15}}\right)\left(\frac{P}{s}\right)^{-3} \mathrm{~W}
$$

implying that for a pulsar with $P=1 \mathrm{~s}$ and $\dot{P}=10^{-15} \mathrm{~s} / \mathrm{s}$, the spin-down luminosity is $3.95 \times 10^{24} \mathrm{~W}$ which is $\sim 0.01 \mathrm{~L}_{\odot}$.

\subsubsection{Magnetic field strength}

It is believed that pulsars have strong dipole magnetic fields. However, a direct measurement for the magnetic field of a pulsar is difficult to obtain. A measurement of magnetic field can be made from cyclotron absorption lines in the spectrum of X-ray binaries (Truemper et al., 1978; McLaughlin et al., 2007). We estimate the magnetic field theoretically using simple physics. From classical electrodynamics, assuming energy loss by a rotating magnetic dipole with a magnetic moment $\vec{m}$ as (Jackson, 1962), we can write

$$
\dot{E}_{\text {dipole }}=\frac{2}{3 c^{3}} m^{2} \Omega^{4} \sin ^{2} \alpha
$$

where $\alpha$ is the angle between the magnetic axis and the rotation axis and $c$ is the speed of light. By equating the spin-down luminosity with $\dot{E}_{\text {dipole }}$, we derive an expression for the rotational frequency evolution

$$
\dot{\Omega}=-\left(\frac{2 m^{2} \sin ^{2} \alpha}{3 I c^{3}}\right) \Omega^{3} .
$$


The magnetic moment is approximately equal to the magnetic field strength in the form of $B \approx m / r^{3}$, so that we get the surface magnetic field strength

$$
B_{\text {surf }}=B(r=R)=\sqrt{\frac{3 c^{3} I}{8 \pi^{2} R^{6} \sin ^{2} \alpha} P \dot{P}}
$$

For a typical NS with $I=10^{38} \mathrm{~kg} \mathrm{~m}{ }^{2}$ and radius of $R=10 \mathrm{~km}$, we find

$$
B_{\text {surf }} \simeq 10^{8}\left(\frac{\dot{P}}{10^{-15}}\right)^{1 / 2}\left(\frac{P}{s}\right)^{1 / 2} \mathrm{~T}
$$

for an orthogonal rotator, $\alpha=90^{\circ}$. With measured $P$ and $\dot{P}$, the inferred surface magnetic fields of observed pulsars are $\sim 10^{4}-10^{10} \mathrm{~T}$.

\subsubsection{Age estimate}

The rotational frequency evolution (Equation 1.18), more generally can be written as a power law, in terms of pulse frequency $\nu$, so that

$$
\dot{\nu}=-K \nu^{n}
$$

where $n$ is known as the braking index and $K$ is a constant. For a pure magnetic dipole, the braking index is $n=3$ (see Equation 1.18). Thus, the evolution of the pulse period $(\nu=1 / P)$ of the pulsar becomes $\dot{P}=K P^{2-n}$. By integrating this first-order differential equation, we can derive an expression for the age of the pulsar

$$
T=\frac{P}{(n-1) \dot{P}}\left[1-\left(\frac{P_{0}}{P}\right)^{n-1}\right]
$$


where $P_{0}$ is the spin period at birth. By using the observed $\ddot{\nu}$ for some pulsars, braking indices have been measured. Currently, the values range from $n=0.9$ to $n=2.9$ (Hamil et al., 2015), implying that the actual value is less than the pure dipole braking index of $n=3$. However, for simplicity, $n=3$ is assumed in most pulsar studies. Assuming that the initial spin period is much shorter than the present value $\left(P_{0} \ll P\right)$ and the spin-down is due to magnetic dipole radiation $(n=3)$, Equation (1.22) simplifies to the characteristic age of

$$
\tau_{c}=\frac{P}{2 \dot{P}} \simeq 15.8\left(\frac{P}{s}\right)\left(\frac{\dot{P}}{10^{-15}}\right)^{-1} \mathrm{Myr} .
$$

This equation gives an approximate value for the age of a pulsar based on its period and period derivative. For instance, the characteristic age of the Crab pulsar is 1240 yr, but the true known age is about 950 yr. Therefore, this is a crude estimation (Hanson, 1979) and cannot be used as a reliable estimate of the true pulsar age. This is especially true for milliseconds pulsars that are believed to be recycled by a binary companion (Alpar et al., 1982).

\subsection{5 $P-\dot{P}$ diagram of pulsars}

As discussed above, pulsar properties such as spin-down luminosity, magnetic field strength, and characteristic age are obtained from the inferred period and period derivative. Therefore, we can present these properties and the location of the pulsar in period versus period-derivative space. This plot is known as $P-\dot{P}$ diagram and is shown in Figure 1.9. 


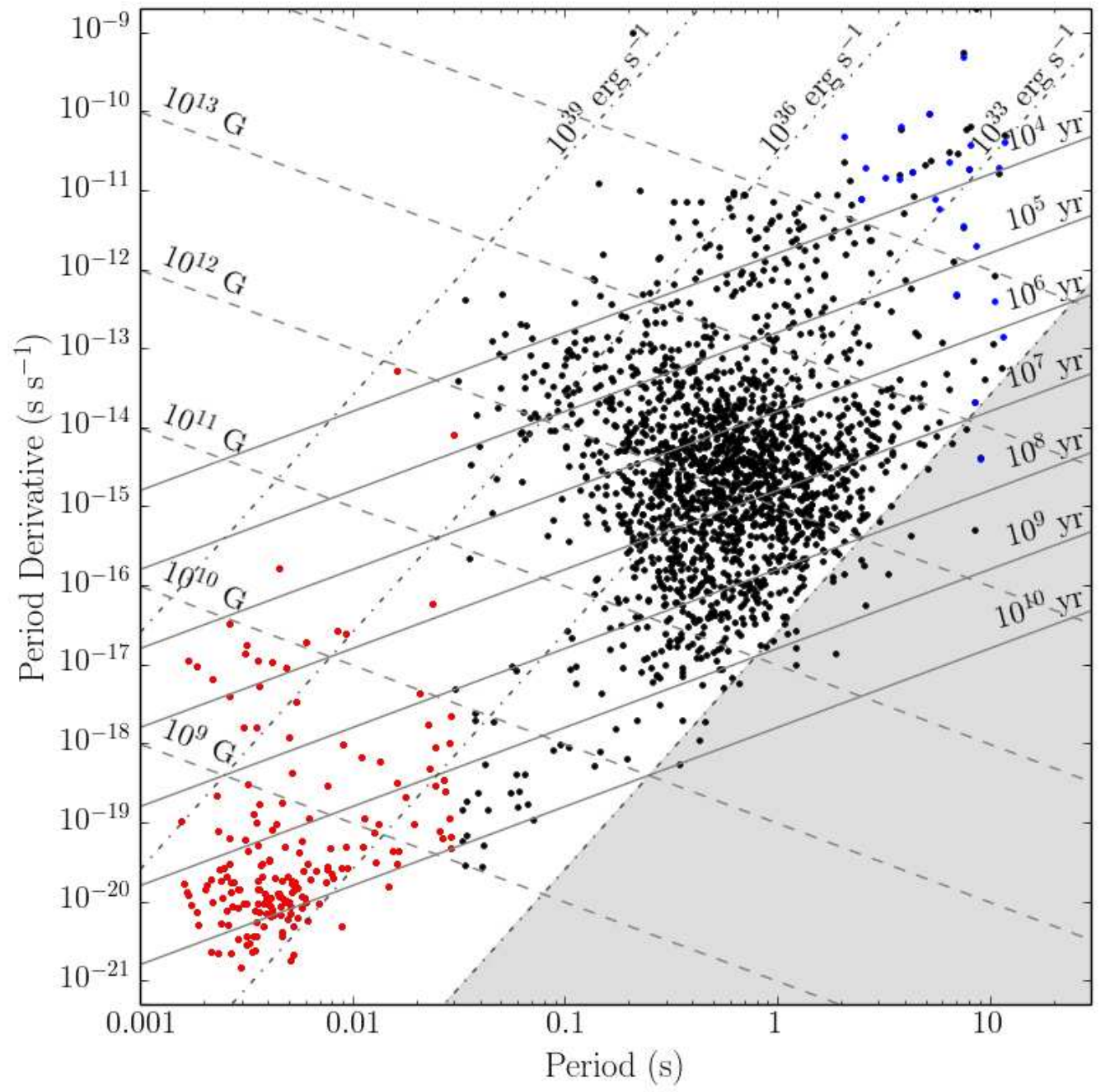

Figure 1.9: $P-\dot{P}$ diagram for known pulsars. Note that the two axes are in logarithmic values. The different lines correspond to lines of constant magnetic field, characteristic age and spin-down luminosity. The shaded region is the so called "pulsar graveyard" where pulsars stop emitting radio pulses. The blue dots indicate magnetars while the red dots indicate millisecond pulsars. 
According to the $P-\dot{P}$ diagram, we can classify radio pulsars mainly into two categories, namely canonical pulsars $(\mathrm{CPs})$ that have periods of $\sim 1 \mathrm{~s}$ and fast spinning millisecond pulsars (MSPs). Most of the MSPs are in binary systems with a companion star. This population is very old with characteristic ages of $>100$ Myr. We believe that MSPs were normal pulsars and then were recycled from their binary companion. Thus, during the recycling process, the old normal pulsar accretes materials from the companion star (Alpar et al., 1982; Tauris et al., 2012). This process transfers the angular momentum to the pulsar and spins it up to millisecond periods. The fastest spinning known MSP is PSR J1748-2446ad (a binary MSP located in the globular cluster Terzan 5) that has a spin period of $1.396 \mathrm{~ms}$ (Hessels et al., 2006). There is another class of neutron stars that has been identified in the last two decades. These so called "magnetars" are neutron stars with magnetic fields of the order of $\sim 10^{10} \mathrm{~T}$ (Mereghetti et al., 2015, and references therein). These stars are assumed to be young energetic neutron stars that lose their energy via spin-down torque and decay of magnetic field. These are believed to be sources of soft gamma-ray repeaters (see sec. 1.8.2.1 for more details.).

As pulsars get older, they slow down and their ability to produce coherent radio emission also decreases. The precise time at which the turnoff occurs depends on both the structure and magnitude of the star's magnetic field. This has led to calculation of theoretical "death lines" in the $P-\dot{P}$ diagram, after crossing which pulsars become radio-quiet. Bhattacharya et al. (1992) have approximated the death 
line as,

$$
\frac{B}{P^{2}}=0.17 \times 10^{-2} \mathrm{~T} \mathrm{~s}^{-2},
$$

where $B$ is the magnetic field and $P$ is the period. This theoretical approximation has been well observed empirically, which can be seen as a well defined cutoff in the $P-\dot{P}$ diagram.

\subsubsection{Dispersion measure (DM)}

Pulsar electromagnetic radiation propagates through the cold ionized plasma in the ISM before it reaches the Earth. The propagation causes a frequency dependent delay in the reception of these waves at Earth because of the dispersion of waves in the ISM. This phenomenon occurs due to the fact that the refraction index of the ISM (Eq. 1.2) is frequency dependent. The group velocity of the wave is $v_{g}=c \mu$, which is less than the speed of light. Therefore, we can obtain the time delay of the signal through propagation in the ISM between the pulsar and the Earth (for distance $d$ ). The time delay,

$$
t=\left(\int_{0}^{d} \frac{d l}{v_{g}}\right)-\frac{d}{c}
$$

By performing the integration and assuming $f_{p} / f \ll 1$, we get an expression for the time delay, $t=D \times \mathrm{DM} / f^{2}$, where the dispersion constant $D=e^{2} / 2 \pi m_{e} c$ and the dispersion measure 


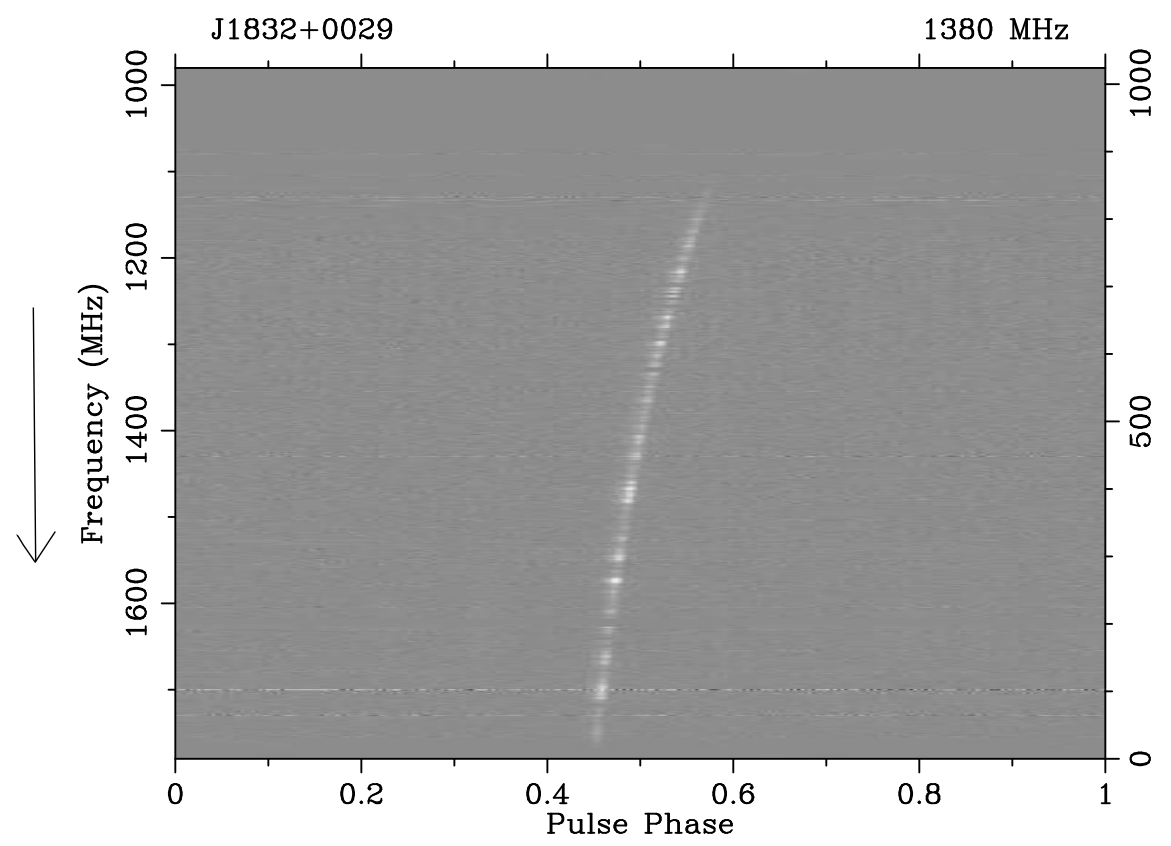

Figure 1.10: Dispersion of the radio signal across the frequency band for PSR J1832+ 0029. Note that the pulses at high frequency reach the observer earlier than the pulses at low frequency. One can see the lack of emission at the edge of the band due to the receiver rolloff.

$$
\mathrm{DM}=\int_{0}^{d} n_{e} d l,
$$

with units of $\mathrm{cm}^{-3}$ pc. Thus, the time delay depends on the observing frequency, so that high frequency signals from the pulsar reach the Earth earlier than low frequency signals (Figure 1.10). The time delay between two frequencies $f_{1}$ and $f_{2}$ (in $\mathrm{MHz}$ ) is

$$
\Delta t \simeq 4.15 \times 10^{6} \times\left(f_{1}^{-2}-f_{2}^{-2}\right) \times\left(\frac{\mathrm{DM}}{\mathrm{cm}^{-3} \mathrm{pc}}\right) \mathrm{ms} .
$$

From a measurement of DM, one can infer the distance to the pulsar if the electron density along the line of sight to the pulsar is well known. By modeling 
Galactic structure, Cordes \& Lazio (2002a) developed an electron density model that can be used to obtain $n_{e}$ along a given line of sight and then estimate the distance to the pulsar. Very recently, Yao et al. (2017) have come up with a new electron density model for the Galaxy where they also include models for the Magellanic clouds and the intergalactic medium.

\subsubsection{Spectral Index}

The flux density spectrum of a pulsar at radio wavelengths can be characterized by a power-law so that the measured flux of the pulsar at some frequency $\nu$,

$$
S_{\text {meas }, \nu} \sim k \nu^{\alpha},
$$

where $\alpha$ is the spectral index. The spectral indices of pulsars range from -0.5 to -3.5 with a typical value of -1.4 (Bates et al., 2014). One of the very first studies of pulsar spectral indices was done by Sieber (1973). The author observed that pulsar spectra showed various kinds of behavior. Some spectra were flat while some showed a broken power-law though most of them followed the single power-law model. The author also discussed some spectra that showed a turnover at frequencies of $\sim$ $100 \mathrm{MHz}$, which might be explained by synchrotron self-absorption and thermal absorption. Kijak et al. (2007) discovered a new class of pulsars that showed a turnover in their flux density spectrum at frequencies of $\sim 1 \mathrm{GHz}$. Only a handful of these so-called "Giga hertz peaked spectra" (GPS) pulsars are known (Kijak et al., 2007, 2011; Dembska et al., 2014). The reason for their turnover can be attributed 
to thermal absorption of radio waves in dense, ionized plasma along the line of sight. Figure 1.11 shows different types of pulsar spectra observed.

\subsection{The pulsar magnetosphere}

We still do not have a clear understanding of the pulsar magnetosphere. There are a few models put forward to explain the structure, but none of these models can explain all the observed characteristics. However, the current understanding of the NS magnetosphere and its characteristics are capable of explaining some main features of the observed electromagnetic radiation such as pulse profile shapes, linear polarization characteristics, and pulsar geometry in general. Here, we mainly discuss the theoretical background of pulsar magnetospheres.

Based on their investigation, Goldreich \& Julian (1969) proposed a simplified, but useful, model to explain pulsar electrodynamics. Following their discussion, a NS can be idealized as a completely conducting, sharply bounded sphere, rigidly rotating in vacuum and is an aligned rotator (i.e. the magnetic axis is aligned with the rotation axis) with a spin angular velocity $\Omega$. They assumed that the NS has a dipolar magnetic field and the NS is an excellent electrical conductor. Therefore, with rotation, the star will be polarized and the surface charge density is quadrupolar, so that the outside electric field is a quadrupole (see Michel, 1991). The electric field component that is parallel to the magnetic field line on the NS surface 

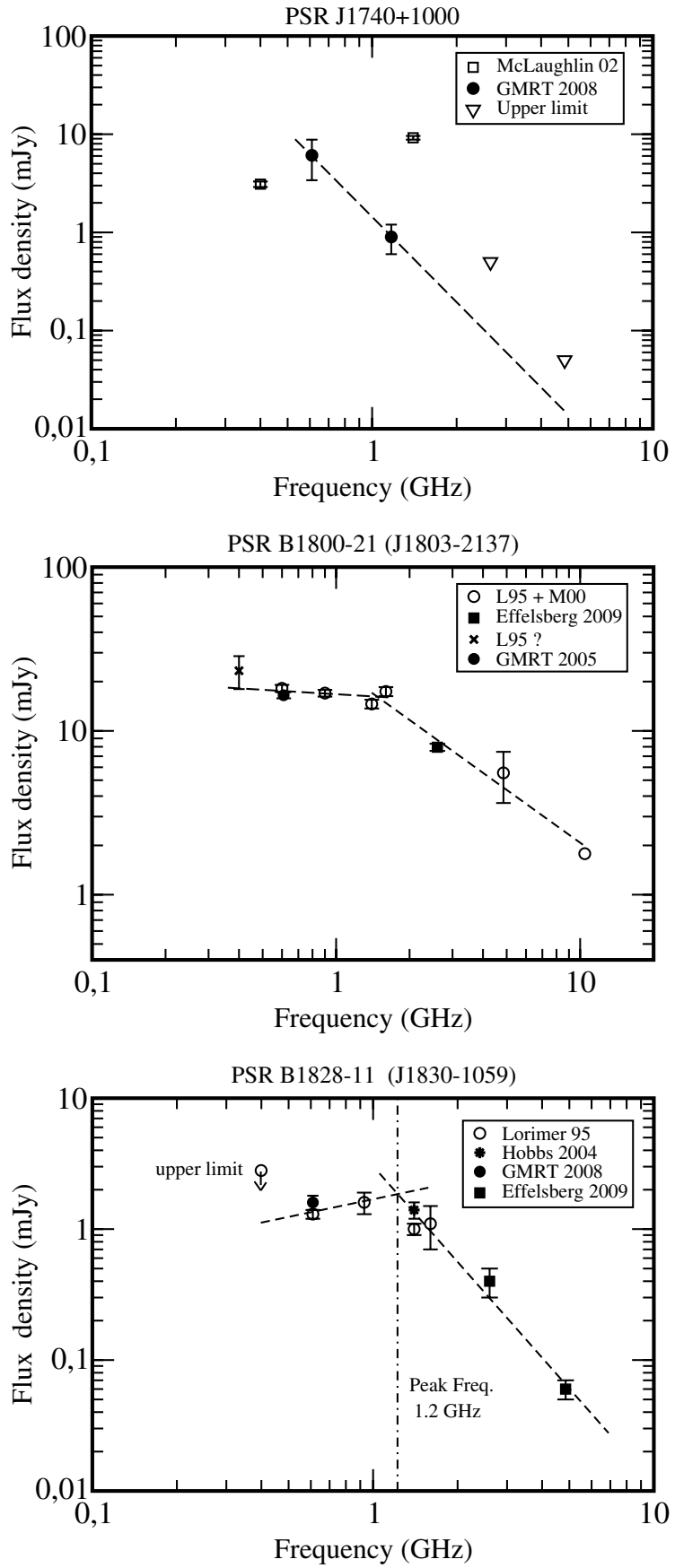

Figure 1.11: Different shapes of flux density spectrum of pulsars. Figure taken from (Kijak et al., 2011). 


$$
E_{\|}(r=R)=\left.\frac{\vec{E} \cdot \vec{B}}{B}\right|_{r=R}=-\frac{\Omega B_{0} R}{c} \cos ^{3} \theta
$$

where $B_{0}$ is the polar magnetic field, $R$ is the NS radius, $c$ is the speed of light, and $\theta$ is the polar angle. The outward electric force $\left(F=q E_{\|}\right)$exerted on the charged particles on the NS surface is much greater than the inward gravitational force on them. Therefore, these charged particles leave the NS surface and enter the magnetosphere. Thus, the assumed vacuum condition outside the NS no longer exists, but rather the magnetosphere is filled with a dense plasma with a local charge distribution

$$
\rho_{e}(r, \theta)=\frac{\vec{\nabla} \cdot \vec{E}}{4 \pi}=-\frac{\vec{\Omega} \cdot \vec{B}}{2 \pi c}=-\frac{B_{0} \Omega R^{3}}{4 \pi c r^{3}}\left(3 \cos ^{2} \theta-1\right) .
$$

Once this charge distribution is arranged in the magnetosphere, the parallel component of the electric field to the magnetic field becomes zero $(\vec{E} \cdot \vec{B} \sim 0)$. In other words, the parallel electric field component is screened from these particles and a force-free condition is maintained outside the star. With the above charge distribution, it is clearly seen that the charges above the equatorial region and the poles are opposite in sign, whereas $\rho_{e}$ changes sign when the polar angle $\theta$ is at $\cos \theta=1 / \sqrt{3}$. This surface is called as the null-charge surface, where $\vec{\Omega} \cdot \vec{B}=0$. Figure 1.12 shows the particle distribution in the magnetosphere according to Goldreich \& Julian (1969). The particle number density at the magnetic pole on the NS surface $n_{G J}=7 \times 10^{10} P^{-0.5} \dot{P}^{0.5}$ particle $\mathrm{cm}^{-3}$, where $P$ in seconds and $\dot{P}$ in units of $10^{-15}$, is known as the Goldreich-Julian (GJ) density. 


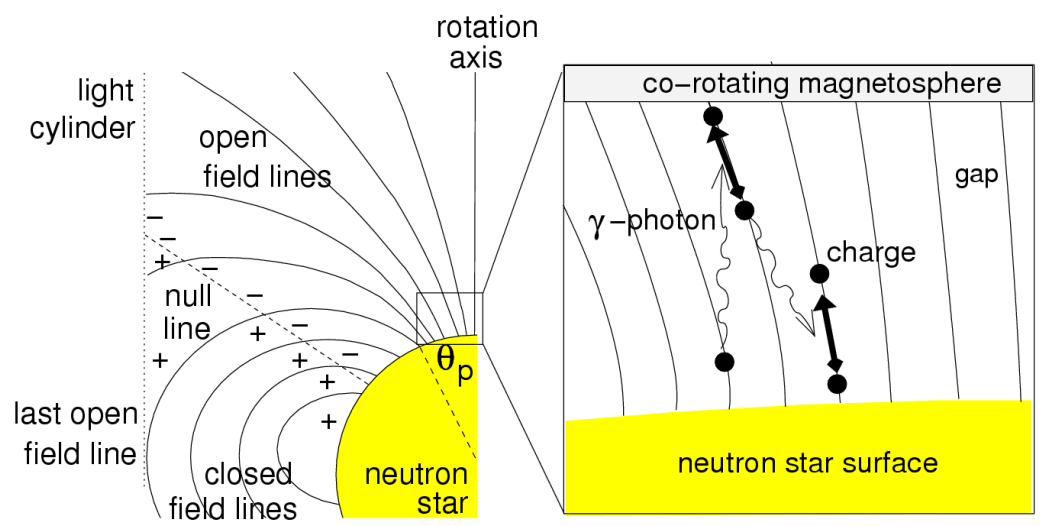

Figure 1.12: Pulsar magnetosphere in the Goldreich-Julian model. The pair creation in the polar gap is indicated on the right (Lorimer \& Kramer, 2005).

The drift velocity $V_{D}=(\vec{E} \times \vec{B}) / B^{2}$ on charged particles is in the azimuth direction $\hat{\phi}$, which forces them to corotate with the NS. This corotation is limited up to the light cylinder radius, where the corotational velocity reaches the speed of light, $R_{L C}=c P / 2 \pi$. This is the boundary of the magnetosphere. The magnetic field lines that are closed within the light cylinder (LC) are referred to as closed field lines and the field lines that do not close are known as open field lines. The polar cap (PC) region is defined by the locus of the foot of the last closed field lines (i.e. the field lines that just touch the LC) on the NS surface. Therefore, the base of all the open field lines is located inside the polar cap region.

With this model, the particles corotate in the magnetosphere. However, above the polar cap, the corotating particles can flow out from the LC along the open field lines due to the centrifugal force of the rotating neutron star. Therefore, the particle density drops from the GJ density and the previously screened $E_{\|}$component is no longer zero $(\vec{E} \cdot \vec{B} \neq 0)$. This breaks the force-free condition in the magnetosphere. 
Thus, the charged particles accelerate with the $E_{\|}$component along open magnetic field lines and produce electromagnetic radiation.

In general, there are few regions located in the pulsar magnetosphere in which the GJ density condition is exceeded. In these regions, the particle depletion occurs and $E_{\|}$cannot be screened. These regions are called acceleration gaps. Mainly, the electromagnetic radiation is generated with these gaps. The commonly identified gaps are the polar gap (or the inner magnetosphere gap) and the outer magnetosphere gap (see Figure 1.7). The radio emission is believed to originate from the polar cap (Ruderman \& Sutherland, 1975) while the high energy emission originates in the outer gap (Cheng et al., 1986).

\subsubsection{Pulsar radio emission}

Observations show that radio pulsars have small duty cycles (0.01-0.1), i.e narrow widths compared to the pulse period (Rankin, 1990). Therefore, the radio emitting region is likely to be localized to a narrow region in the magnetosphere. Although the radio emission mechanism is not completely understood, there are models to explain the observed emission. For an observed flux of the pulsar at a frequency $\nu, F_{\nu}$, we can define a brightness temperature $T_{b}$ such that,

$$
T_{b}=\frac{F_{\nu} 4 \pi c^{2} d^{2}}{k_{b} \nu^{2} r^{2}}
$$

where $k_{b}$ is the Boltzmann constant, $r$ is the radius of the emitting region and $d$ is the distance to the observer. The brightness temperature is the physical temperature 
of the source, if it were emitting thermal radiation isotropically in space. Plugging in typical values for $F_{\nu}=1 \mathrm{Jy}$ where $1 \mathrm{Jy}=10^{-26} \mathrm{~W} \mathrm{~m}^{-2} \mathrm{~Hz}^{-1}, \nu=1.4 \times 10^{9} \mathrm{~Hz}$, $r=c \Delta t$ where $t=10 \mathrm{~ms}$ is the pulse width and $d=1 \mathrm{kpc}$, we obtain $T_{b} \approx 10^{25} \mathrm{~K}$. In order to obtain the observed high brightness temperature of pulsar radio emission, the radiation has to be generated coherently. We primarily discuss the antenna mechanism based on pulsar polar cap geometry as the radio emission mechanism (Ruderman \& Sutherland, 1975).

According to the proposed mechanism, the charged particles are bunched to generate radiation coherently. The basic idea is that if $N$ particles of charge $q$ are confined in a volume of size less than half of the emitted wavelength, then they will radiate in phase like a particle of charge $N q$. Then, the emitted power is $N^{2}$ times the power radiated by one single particle. The most common radio emission mechanism was proposed by Ruderman \& Sutherland (1975) involving a PC model in which the radio emission is generated from the accelerated particles within the polar gap. The polar gap is a charge depleted region located within the polar cap bounded by the last closed field lines and extends upwards from the NS surface. When charged particles leave the magnetosphere through the LC the polar gap is formed. Within the gap, the force-free condition is violated $(\vec{E} \cdot \vec{B} \neq 0)$, but the rest of the magnetosphere is force-free. The parallel component of the electric field at the pole within the gap,

$$
E_{\|}=2 \frac{\Omega B_{s}}{c}(h-z),
$$


where $B_{s}$ is the surface magnetic field, $h$ is the polar gap height, and $z$ is the distance from the NS surface. Therefore, the potential drop across the gap,

$$
\Delta V=\frac{\Omega B_{s} h^{2}}{c} .
$$

These charged particles are known as "primary particles". They accelerate through non-zero electric field component in the gap and gain extremely relativistic energies. For a pulsar with $B_{s}=10^{8} \mathrm{~T}, \Omega=2 \pi \mathrm{s}^{-1}$, and $h=10^{2} \mathrm{~m}$, the energy becomes $e \Delta V \geq 10^{11} \mathrm{eV}$. When these particles accelerate along magnetic field lines, curvature radiation $(\mathrm{CR})$ is emitted with photon energies

$$
E_{p h}=\hbar \omega=3 \gamma^{3} \hbar c / 2 r_{c},
$$

where $\gamma$ is the Lorentz factor and $r_{c}$ is the radius of curvature. When a CR photon exceeds the energy $2 m c^{2}$, a $e^{+}-e^{-}$pair is formed (known as pair cascade), where $m$ is the particle mass (see Figure 1.13). This pair can discharge the gap and significantly change $E_{\|}$. Due to the discharge, a pair formation front occurs at a height $h$ and is the upper boundary of the gap. The newly generated pairs are so called "secondary particles" and have low energies compared to those of primary particles. These secondary particles then move to the force-free region in the magnetosphere above the polar gap and travel with a constant velocity along the magnetic field line.

The primary charged particles have energies that correspond to radiation in the gamma-ray regime. However, the secondary particles have much lower energies and the CR frequency becomes $\omega_{c} / 2 \pi=10^{9} \mathrm{~Hz}$, which is in the radio band. Therefore, 


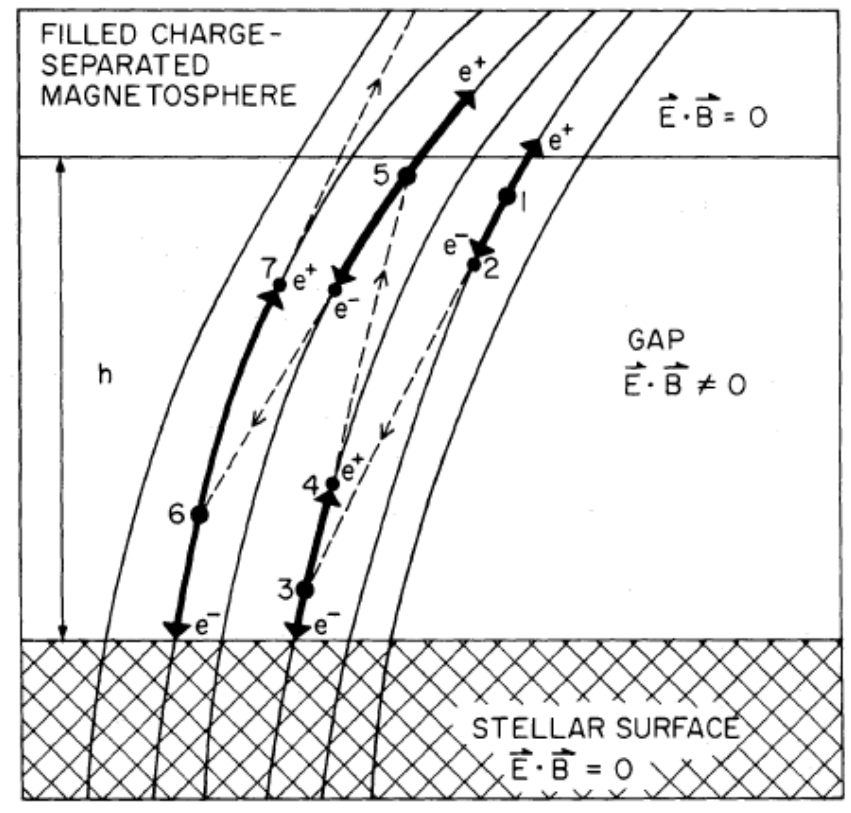

Figure 1.13: Pair cascade in the polar gap of the pulsar magnetosphere. A primary particle produced photon discharges to an $e^{+}-e^{-}$pair at 1 . Then the secondary $e^{+}$ enters to the force-free magnetosphere above the gap and $e^{-}$accelerates toward the surface and radiates a $\mathrm{CR}$ photon at 2. This photon again discharge to a pair at 3. This process continues until the CR photons do not have enough energy to produce pairs having energy $<2 m c^{2}$ (Ruderman \& Sutherland, 1975). 
the secondary particles are believed to produce radio emission in pulsars.

\subsubsection{High-energy emission}

Along with radio emission, the movement of relativistic charged particles can also lead to emission of higher energy photons depending on the Lorentz factor of the plasma and the potential drop in one of the gaps in the magnetosphere. A few pulsars have been observed to emit X-rays and gamma-rays (Hermsen et al., 2013; Abdo et al., 2013)

A few models that explain high energy emission are: (a) curvature radiation; (b) synchrotron radiation; (c) inverse compton scattering (ICS). As explained earlier, high energy emission, like radio emission is possible in the gaps regions of the magnetosphere where high energy plasma creation is feasible. Two such regions have been identified: (1) the outer gap near the light cylinder along the open field lines; and (2) the polar gap region that is believed to be responsible for radio emission (see Figure 1.7 for details).

Ruderman \& Sutherland (1975), Sturrock (1971) and Arons \& Scharlemann (1979) were the first to propose that the particles are accelerated in the induced field near the PC to produce curvature radiation which creates a pair plasma in the strong magnetic field. This pair is responsible for partially screening the electric field and in this process, some of the positrons produced are accelerated towards the PC thus heating up the surface which can be observed as X-rays. Later, it was realized that ICS can cause the creation of secondary plasma that can cause the 
observed X rays by PC heating (Harding \& Muslimov, 2002). It was found that for ICS to be a dominant process for $E_{\|}$screening, surface temperatures should be higher than $10^{6} \mathrm{~K}$. Neutron stars are also known to emit continuum X ray emission from the surface (Becker \& Truemper, 1997, and references therein).

Over the last few decades, many pulsars have been discovered at multiple wavelengths. In some pulsars, a correlation between the radio and higher energy emission has been observed which makes us believe that both types of emission emanate from a similar region in the magnetosphere which favors a PC gap model for both modes of emission for most pulsars.

\subsection{Emission geometry and characteristics}

As shown in Figure 1.8, pulse profiles of pulsars have different shapes, varying from a single peak to several components. By analyzing various pulse profile shapes with their geometries, Rankin (1983a,b, 1990, 1993) proposed that the pulsar radio beam can be understood as a core and a set of conal components. Then the observed pulse profile shape depends on the beam structure and the relative motion between the line-of-sight and the radio beam. This results in different profile shapes according to which section of the beam structure our line-of-sight encounters, with the pulsar rotation (see Figure 1.14). However, some complex profile shapes cannot be explained with this simple core and conal beam structure. Therefore, Lyne \& Manchester (1988) proposed that pulsar beam may have a random patchy structure, so that the flux is larger in some patches. These two beam models are widely used 


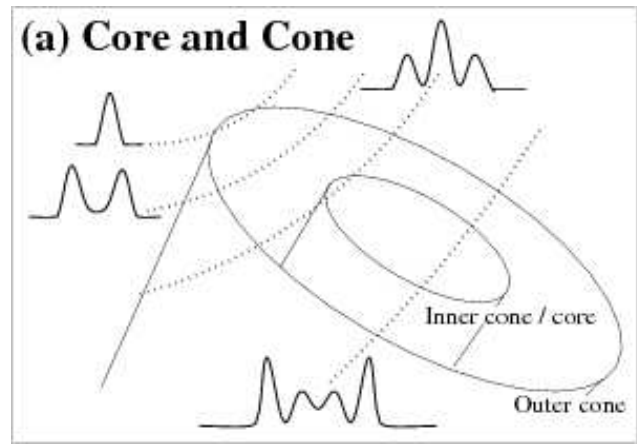

\section{(b) Patchy Beams}

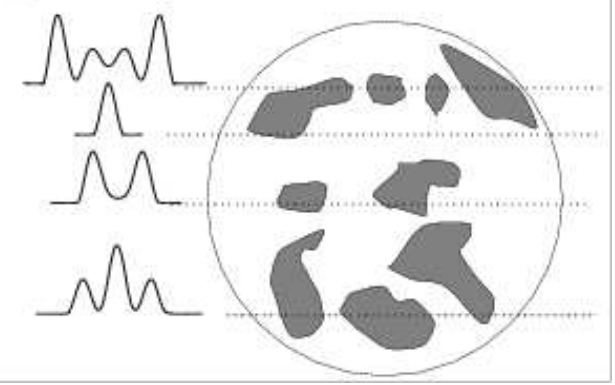

Figure 1.14: Schematic views of different beam structures. Dashed lines are different lines of sight across the beam and resultant pulse profiles are shown for (a) core and conal beam structure (b) patchy beam structure (Lorimer \& Kramer, 2005).

in explaining pulse profiles and time-evolving pulse shapes of pulsars.

Though most averaged pulsar profiles are stable over a long timescale, pulsars exhibit high emission variability over shorter timescales. Figure 1.15 shows single pulses from a pulse sequence of PSR B0826-34 where it clearly demonstrates the variability of single pulses. These variations manifest themselves as observed phenomena of nulling; the abrupt cessation of radio emission for a few or more pulse periods (Gajjar et al., 2012) and mode-changing; a sudden change in the integrated pulse profile due to the reorganization of radio beams in the magnetosphere (Rankin, 1983). Nulling and mode-changing have been observed in about 200 pulsars to date (Wang et al., 2007; Gajjar et al., 2012). Observations of these phenomena helps us to gain insights into the underlying emission physics of the pulsar magnetosphere. Lyne et al. (2010) observed a correlation between mode-changing and the rate of spin-down in a sample of pulsars suggesting that mode-change is a result of a global change in the emission region of the neutron star while nulling can be thought of as a local change. Kramer et al. (2006) discovered a new class of pulsars that show an extreme form of nulling. These "intermittent" pulsars are 
PSR B0826-34 at $318 \mathrm{MHz}$ using the GMRT

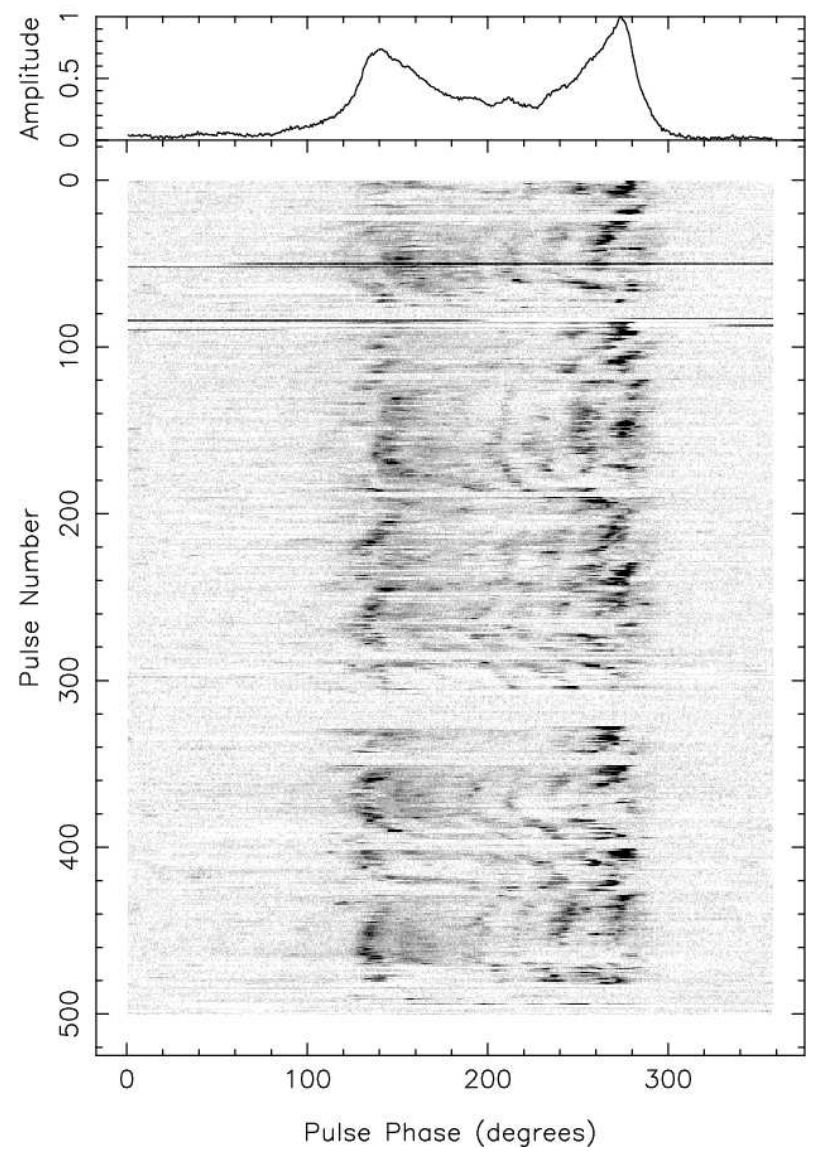

Figure 1.15: Single pulse train of PSR B0826-34. One can identify nulls in the timeseries with bright emission in most pulses resulting in a very different looking averaged profile compared to the single pulses in the top panel. The emission in the single pulses is also variable with drift patterns seen within them. Figure taken from Gupta et al. (2004). 
known to remain radio-quiet for a couple of years before becoming detectable again. Only five intermittent pulsars are known to date (Kramer et al., 2006; Lorimer et al., 2012; Camilo et al., 2012; Lyne et al., 2017). More and more discoveries of such pulsars will help in revealing the exact nature of the physics responsible for emission variability in pulsars.

\subsection{Galactic pulsar population}

To date we have discovered 2613 pulsars ${ }^{1}$. However, with our current technology, we are most sensitive to pulsars in the close vicinity of the Sun though there are pulsars found in the LMC and the SMC. The observational bias is evident in the projection of detected pulsars on the Galactic plane (Figure 1.16) which shows that the known pulsars are clustered around the position of the Sun. Moreover, since we only detect bright pulsars, the population sampled by us has an inherent bias towards the brighter tail of the underlying luminosity function which makes inferring properties of a generic pulsar population very difficult.

After the discovery of pulsars, it was difficult to get a handle on the total population of these sources as we did not have enough information about their periods, period derivatives, magnetic fields and other physical parameters. The Parkes multi-beam pulsar survey (Manchester et al., 2001) was one of the most successful surveys that increased the number of detected pulsars by a factor of 21. After the survey results were published, Faucher-Giguère \& Kaspi (2006) were the first

\footnotetext{
${ }^{1}$ Data taken from the ATNF Pulsar Catalog http://www.atnf.csiro.au/people/pulsar/ psrcat/
} 


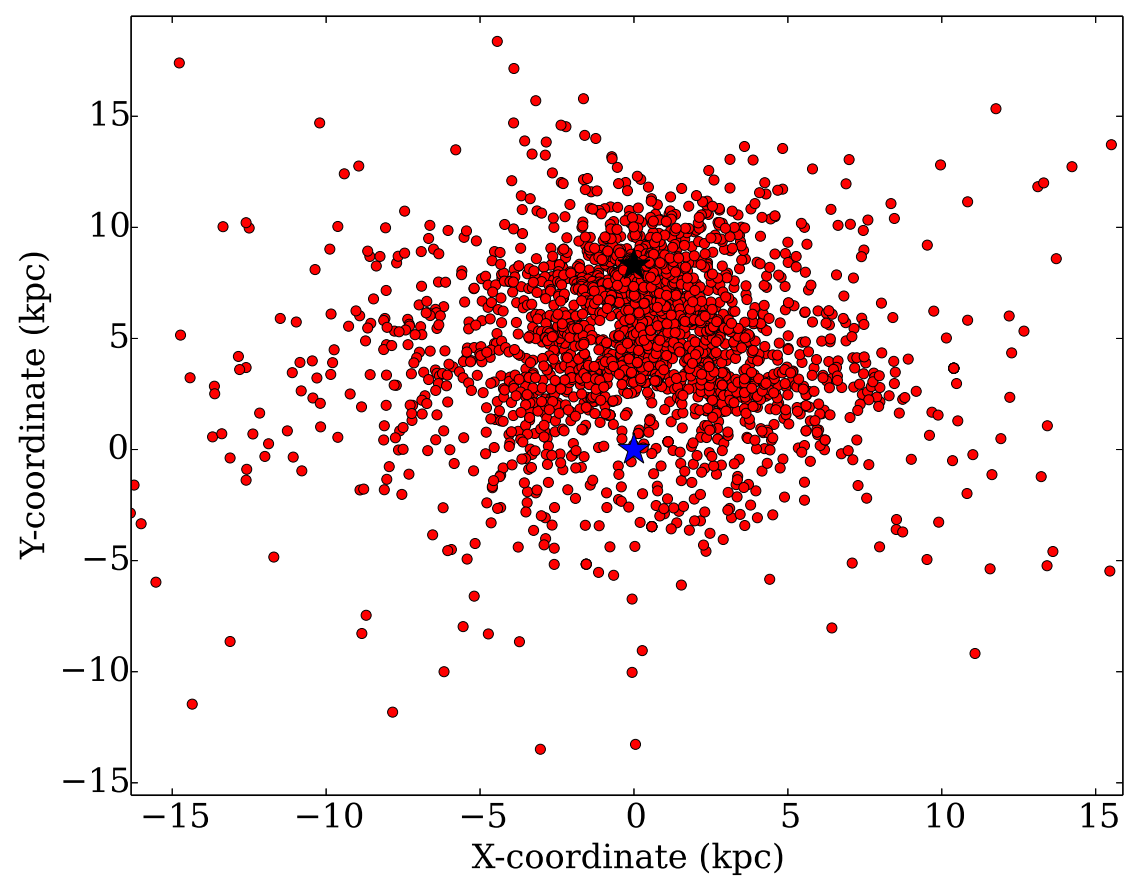

Figure 1.16: Distribution of known pulsars (red dots). The black star is the position of the Solar system and the blue star shows the position of the Galactic Center.

group to attempt a detailed population study of pulsars. Using likelihood analysis, (Faucher-Giguère \& Kaspi, 2006) were able to constrain the underlying luminosity function of isolated pulsars. They estimated a birth rate of 2.8 pulsars per century and suggested that there are $1.2 \times 10^{6}$ pulsars in the Galaxy with $10 \%$ of them beaming towards us. We should note that in all these analyses, the estimates are reported for certain luminosity cut-off that should be taken into account before making any inferences about the overall characteristics of the Galactic pulsar population. Nevertheless, the results suggest that we have sampled only $\sim 0.2 \%$ of the total population. 


\subsubsection{Pulsars in the Galactic Center}

The lack of pulsars in the Galactic Center (GC) region of our Milky-Way has been a puzzle for pulsar astronomers. Although high-sensitivity surveys have revealed a number of highly dispersed pulsars in the inner Galaxy, none have so far been found in the Galactic Center (GC) region, which we define to be within a projected distance of $1 \mathrm{pc}$ from Sgr A*. Understanding stellar populations in the GC region and their interactions with the central supermassive black hole is one of the key science goals in modern astronomy. The high stellar densities in the GC preclude the existence of a significant radio-loud pulsar population. Motivated by the promise of finding pulsars in the GC, there have been a number of surveys in the last decade towards the GC and none of them have found a single pulsar. The discovery of a magnetar very close to SgrA* (Mori et al., 2013) brought attention back to the missing pulsar problem. This discovery led a number of authors to conclude that there is a genuine dearth of pulsars in the GC and that the GC environment most likely favors magnetar creation over pulsars (Chennamangalam \& Lorimer, 2014; Dexter \& O'Leary, 2014). Moreover, the GC environment was assumed to be a hyperstrong scattering environment which would render any pulsar undetectable at frequencies of $\sim 1.4 \mathrm{GHz}$ (Lazio \& Cordes, 1998) but recent results by (Bower et al., 2014) have shown that scattering screen is actually $6 \mathrm{kpc}$ away from the GC and thereby would not cause strong scattering along the line of sight. This result makes the lack of detection baffling.

The presence of hot, ionized gas in the central parsec of our Galaxy (Pedlar 
et al., 1989; Gillessen et al., 2012) raises the question of whether absorption can affect detection of radio pulsars. Recent studies have shown free-free thermal absorption to be the primary source of gigahertz peaked spectra, where the flux density spectrum shows a turnover at frequencies of $\sim 1 \mathrm{GHz}$ in some pulsars found in dense ionized environments (Lewandowski et al., 2015; Rajwade et al., 2016a).

Such a dense and highly turbulent environment can also be responsible for large scattering, thereby reducing incoming pulsar radio flux density in our line of sight. The effects of the interstellar medium (ISM) in the GC on pulsar flux densities have been studied previously. Cordes \& Lazio (1997) modeled multi-path scattering in the GC in terms of a thin screen near the center. As a result, the radio pulses observed can be substantially broadened at lower frequencies. Wharton et al. (2012) studied various flux density mitigation effects due to the ISM that can alter the incoming pulsar flux and result in a non-detection.

\subsection{Fast Radio Bursts}

Fast Radio Bursts (FRBs) are millisecond duration, highly sporadic and dispersed radio pulses that follow the same dispersion relation seen in radio pulses from neutron stars (see section 1.3). The origin of FRBs remains an unanswered question since their discovery a decade ago (Lorimer et al., 2007). Of the 21 FRBs published so far, 16 have been found at Parkes (Thornton et al., 2013; Lorimer et al., 2007;

Petroff et al., 2015; Keane et al., 2016; Champion et al., 2016; Ravi et al., 2016), 3 at Molonglo (Caleb et al., 2017), one at Arecibo (Spitler et al., 2014, 2016) and 
one at Green Bank (Masui et al., 2015). With the exception of the Green Bank and Molonglo FRBs, which were detected at $800 \mathrm{MHz}$, all the other FRBs have so far been seen in the 1-2 GHz band. FRB dispersion measures (DMs) are substantially greater than that expected from free electrons in our Galaxy, suggesting that FRBs are extragalactic in origin. This means that FRBs could serve as probes of the cosmological properties like magnetic field and test of Lorentz invariance (Muñoz et al., 2016; Zhou et al., 2014; Kohler, 2016).

\subsubsection{History}

In 2007, while analyzing pulsar search data, a WVU undergraduate student, David Narkevic, under the guidance of Duncan Lorimer, stumbled upon a bright radio pulse. It showed dispersion smear across the radio band similar to the ones shown by pulses from a radio pulsar. After further checks, it was realized that the source only appeared in three of the thirteen beams of the Parkes multi-beam receiver (Staveley-Smith et al., 1996) and hence could not be terrestrial in nature. This led to the first ever detection of a new radio source and it was called the "Lorimer Burst". These findings were reported in Lorimer et al. (2007). After the initial discovery, there were no new detections of such single radio bursts for the next few years. The lack of detections led Burke-Spolaor et al. (2011) and Kocz et al. (2012) to challenge the existing theory for the Lorimer burst being astrophysical in nature. Along the same time, similar bursts were seen at the Parkes radio telescope that showed a similar dispersion smear and were present in all the 13 beams of the multi- 


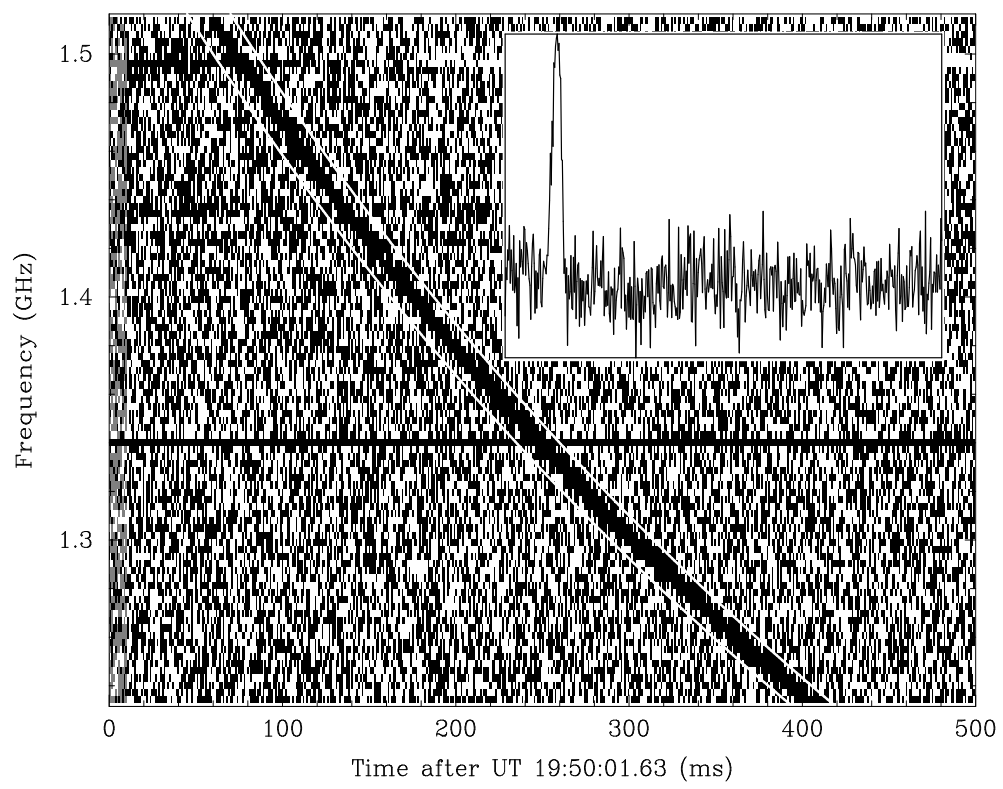

Figure 1.17: Frequency versus time plot showing the dispersed burst. The inset shows the dedispersed frequency collapsed version of the Lorimer burst. Figure taken from Lorimer et al. (2007).

beam receiver suggesting that it was RFI. These bursts showed a dispersion smear similar to the Lorimer Burst and were called "perytons". This brought considerable doubt on the origins of the Lorimer burst and few others that were discovered at the same telescope. Thornton et al. (2013) reported detection of four radio bursts in 2013 and coined the term Fast Radio Bursts. In 2015, a breakthrough came when it was realized that the microwave oven under the Parkes radio telescope caused these perytons and the discovery of a radio burst from different telescope confirmed that the Lorimer burst was after all astrophysical (Petroff et al., 2015). These findings gave rise to immense interest in FRBs and has now developed into a sub-field in radio astronomy by itself. The detection plot of the first FRB is shown in Figure 1.17 . 


\subsubsection{Properties and emission models}

There have been several models to explain emission of FRBs (Lyubarsky, 2014; Lyutikov et al., 2016; Cordes \& Wasserman, 2016; Katz, 2016; Ghisellini, 2017). Discovery of a repeating FRB rules out models that involve destruction of its source though absence of repetition from other sources does not rule out multiple progenitors of FRBs. These include stellar collapse, cataclysmic collisions and merging binaries. Current evidence suggests that FRBs are mostly produced by remnants of stellar collapse, neutron stars or black holes whose deep gravitational well allows for an emission of energy. This postulate includes soft gamma-ray repeaters (SGRs) (Lyubarsky, 2014) or giant pulses from neutron stars (Cordes \& Wasserman, 2016).

The observed pulse widths of ms duration implies a small emitting region of the source. FRBs have extremely high brightness temperatures $\left(\sim 10^{37} \mathrm{~K}\right.$, see Eq. 1.31). Though $T_{b}$ is not the physical temperature of the source, it does mean that a lot of energy is emitted from a region with an area $\mathrm{A} \approx(c \Delta t)^{2}$. Such brightness temperatures have been seen for "nano shots" from Crab pulsar (Hankins et al., 2003). All the evidence points toward coherent emission by charged "bunches" similar to radio pulsars. Since our current knowledge of plasma physics has been unable to unravel the exact emission physics of radio pulsars, the same can be said about FRBs for now. Below, I discuss a few possible progenitors for FRBs. 


\subsubsection{Soft Gamma Ray Repeaters}

SGRs were discovered as transient $\gamma$-ray burst sources that were later found to be persistent X-ray neutron stars (Woods \& Thompson, 2006). The difference in the case of SGRs and radio pulsars is the source energy for the high energy emission, which is the decay of the strong magnetic field. SGRs have been proposed a number of times to explain FRBs (Kulkarni et al., 2014; Lyubarsky, 2014; Katz, 2016). They share a lot of observed properties with FRBs like low duty cycles, high energy output and similar characteristic timescales. These similarities point towards magnetars with high magnetic field as the origin. The excess DM can be contributed by the dense plasma around the young neutron star and the high magnetic energy density can explain the observed brightness temperature.

During one SGR event, $\sim 10^{37}-10^{38} \mathrm{~J}$ of energy is released. Such a high energy requires a source with a very high energy density. Magnetic reconnection in the magnetosphere of neutron stars was the natural model to explain the phenomena. Assuming magnetic reconnection as the source of this energy, one can find the magnetic field of the neutron star surface. Following Thompson \& Duncan (1993), the observed energy has to be confined within the magnetic flux loop hence, the magnetic pressure,

$$
P_{\mathrm{mag}}=\frac{\left(B\left(R_{*}+\Delta R\right)\right)^{2}}{8 \pi} \geq \frac{E_{\mathrm{obs}}}{3 \Delta R^{3}},
$$

where $R_{*}$ is the stellar radius, $E_{\text {obs }}$ is the observed energy and $B(R)=B_{*}\left(R / R_{*}\right)^{3}$ 
where $B_{*}$ is the magnetic field on the NS surface. Using this condition, we find that

$$
B_{*}>4 \times 10^{10}\left(\frac{\Delta R}{10000 \mathrm{~m}}\right)^{-3 / 2}\left(\frac{1+\Delta R / R_{*}}{2}\right)^{3} T .
$$

By assuming that the energy is saturated within the flux tube, one can compute the magnetic field, which is $10^{2}$ times the typical field of pulsars. Hence, young, highly magnetized neutron stars, i.e magnetars, are required to power an SGR event with the observed energies.

Though the physics can explain an FRB event correlated with an SGR event, there are a few difficulties involved. Firstly, the observed X-ray spectrum from an SGR is a black-body spectrum and is heavily self-absorbed at low frequencies which is in contrast to the FRB spectrum that is non-thermal and emitted in the radio regime. Though pulsed radio emission has been observed from magnetars (Camilo et al., 2007), the energy from them is orders of magnitude smaller compared to that of an FRB. Secondly, for magnetic reconnection to occur, strong resistive currents are required. Though the magnetosphere has high energy density, the currents present are not strong enough to make magnetic reconnection plausible (for a review, see Katz, 2016).

\subsubsection{Super-giant pulses from neutron stars}

Since coherent emission is required to explain high brightness temperatures, FRBs have often been related to neutron stars. One postulate is that giant pulses, similar to the ones observed in Crab pulsar could be observed as FRBs if they were 
emitted by a pulsar from a nearby galaxy. Cordes \& Wasserman (2016) did a detailed analysis to check if it would be physically possible for an extragalactic neutron star to emit super-giant pulses so that they could be observed as FRBs. They found that if we compare the energies of nano shots from Crab pulsar to the energy of FRBs, the pulses are weaker by orders of magnitude but there are circumstances in which pulsars would be able to emit stronger giant pulses with energies comparable to observed FRBs. The largest GPs of Crab pulsar are easy to account for in the energy budget of the dipolar radiation model but need a significant amount of charged particles to be directed into coherent emission.

If $\dot{E}$ is the spin-down luminosity then, the maximum GP emission flux,

$$
S_{\nu, \max }=\left(\frac{4 \pi \epsilon_{m}}{\Omega_{r}}\right)\left(\frac{\dot{E}}{4 \pi \Delta \nu_{r} d^{2}}\right),
$$

where the ratio $4 \pi / \Omega_{r}$ is the increase in flux due the relativistic beaming of the charged particles and $\epsilon_{r}$ is the radio efficiency. FRBs will be possible if the emission is highly beamed or has higher radio efficiency than the pulsars in our Galaxy.

Though the energy of GPs can be accommodated by spin-down losses, it might be borderline on the energy limit of relativistic charged particles. For a force free magnetosphere $(\vec{E} \cdot \vec{B}=0)$, the charge density is given by the GJ charge density, $n_{G J}$ (see section 1.5). Hence, the particle energy loss rate,

$$
\dot{E}_{p}=c A_{p c} n_{G J} \gamma m c^{2}
$$


where $\gamma$ is the Lorentz factor, $m$ is the electron mass and $A_{p c}$ is the area of the polar cap region. From our earlier review of the pulsar magnetosphere, we know that the particle loss rate scales as the square root of the spin-down loss. Comparing the numbers to the GP in Crab pulsar, there is a considerable range in $\gamma$ and $\Omega_{m} / 4 \pi$ for higher amplitudes of GPs from pulsars that would make FRBs a possibility.

\subsubsection{Blitzar}

Based on the fact that most FRBs are single, isolated bursts, Falcke \& Rezzolla (2014) came up with a model of a collapsing neutron star to explain the observed fluxes at cosmological distances. According to their model, a neutron star of mass higher than the critical mass of a non-rotating neutron star is formed. Since the star is rotating rapidly, the centrifugal forces will prevent it from collapse. Eventually as the star slows down due to magnetic braking, there will come a point where the rotation is no longer able to sustain the gravitational pull towards the core and the NS will instantly collapse into a black hole. In this sudden collapse, the magnetic energy density in the NS will be released as a coherent burst that can be detected at radio wavelengths. Falcke \& Rezzolla (2014) have termed this scenario a "Blitzar".

For a supermassive neutron star $\left(\sim 2.3 \mathrm{M}_{\odot}\right)$, the relevant timescale of collapse is the free fall time scale given by,

$$
\tau_{\mathrm{ff}}=40\left(\frac{r}{10 \mathrm{~km}}\right)^{1.5}\left(\frac{m}{2.3 \mathrm{M}_{\odot}}\right)^{-0.5} \mu s
$$

(Lehner et al., 2012) where $r$ is the stellar radius and $m$ is the stellar mass scaled to 
the mass of an assumed supermassive neutron star of $2.3 \mathrm{M}_{\odot}$. Within this timescale, an event horizon will form near the surface of the NS. The magnetosphere of the NS is the only part that will not disappear in the collapse as it is well outside the NS radius. The entire magnetic field detaches and reconnects outside the formed event horizon. This will result in a strong magnetic shock wave moving at velocities close to the speed of light. The total power radiated by the shock,

$$
P_{M S}=\eta_{B}\left(\frac{B^{2}}{4 \pi}\right) \frac{V}{\Delta t}
$$

where $\eta_{B}$ is the fraction of magnetic energy that is dissipated, $V$ is the volume of the NS and $\Delta t$ is the burst duration.

Dionysopoulou et al. (2013) show the temporal evolution of ejected magnetic luminosity for the non-rotation case. They suggest that there is a dominant peak for width $\sim 0.1 \mathrm{~ms}$ followed by additional pulses, lower in strength corresponding to the ringdown of the newly formed black hole. The leading few pulses carry most of the transmitted power and contain about $5 \%$ of the available magnetic energy. Using $\eta_{b}=0.05 \eta_{B, 5}$ and assuming that the bulk energy is released in $0.5 \mathrm{~ms}$, we get,

$$
P_{M S} \simeq 4.2 \times 10^{36} \eta_{B, 5} t^{-1}\left(\frac{B}{10^{8} T}\right)^{2}\left(\frac{r}{10 \mathrm{~km}}\right)^{3} \mathrm{~J} \mathrm{~s}^{-1}
$$

where $B$ is the magnetic field and $t$ is the time in milliseconds, which is consistent with the observed energy. 


\subsubsection{Dispersion measures}

First FRBs were identified as astrophysical radio sources since they followed

a $\nu^{-2}$ dispersion relation similar to what is seen in radio pulses from pulsars. Moreover, the extremely high dispersion measure of FRBs suggest that they are possibly extragalactic in nature as the Milky Way contribution cannot account for the entire DM for these events. The possible extragalaactic nature of FRBs opens a new avenue to study the intergalactic medium (IGM hereafter) that permeates between different galaxies. The potential DM contribution for FRBs comes from the IGM and an assumed host which could be another galaxy. The total DM of an FRB,

$$
\mathrm{DM}_{\mathrm{FRB}}=\mathrm{DM}_{\text {host }}+\mathrm{DM}_{\mathrm{IGM}}+\mathrm{DM}_{\mathrm{mw}}
$$

where $\mathrm{DM}_{\text {host }}$ is the DM contribution from the host galaxy and $\mathrm{DM}_{\mathrm{mw}}$ is the $\mathrm{DM}$ contribution of the Milky Way along that line of sight. Using DM $_{\text {IGM }}=1200 z$ where $z$ is the redshift (Inoue, 2004) and substituting this in equation 1.42, we may estimate the redshift of the source. This ties back to the similar analogy we made in section 1.4.6 where one can estimate the distance to the pulsar based on the known electron density along the line of sight. We must note that in order to obtain accurate distance estimates for pulsars and FRBs via DM, one must have a very clear understanding of the ISM and the IGM. 


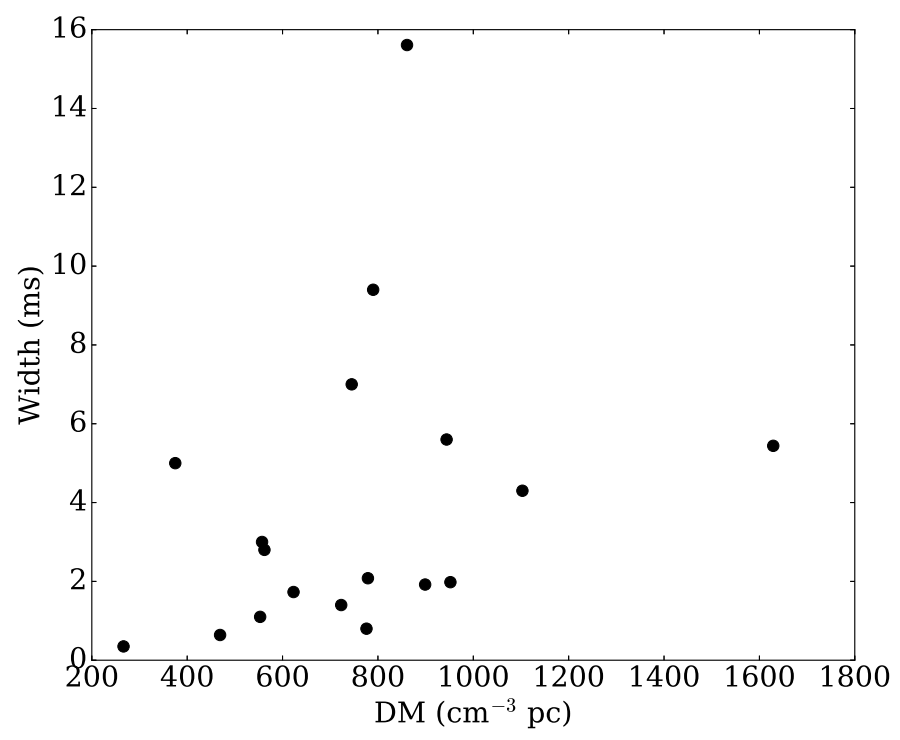

Figure 1.18: Pulse width versus DM for known FRBs. The lack of correlation between the two parameters suggests that for most FRBs, the IGM has lower contribution to scattering for most FRBs. Data taken from frbcat (http: //www . astronomy. swin. edu.au/pulsar/frbcat).

\subsubsection{Scattering and absorption}

All the 20 sources that have been detected so far have shown minimal amount of scatter broadening. Most FRBs have widths of $\sim 1 \mathrm{~ms}$ and a strong dependence of widths with DM suggests that it results from scattering in the IGM. Figure 1.18 shows no correlation between these two parameters meaning that the IGM contributes very little to scattering in most FRBs. Though different lines of sight in our Galaxy do suggest that the scatter broadening could be due to the Milky Way, the most reasonable explanation is that the near source plasma is the major contributor to scattering in FRBs. Cordes \& Wasserman (2016) have done a detailed comparison of scattering observed in FRBs and Galactic pulsars. The authors conclude that either the scattering originates in the host galaxy (25 to $50 \%$ contribution 
to the extragalactic DM) which will lead to overestimation of redshifts for FRBs or the galaxy dominates the extragalactic DM and the pulse broadening, which would mean that the electron density fluctuations in FRB hosts are much smaller compared to ones observed in our Galaxy's ISM. This model favors a scenario where there is a dense plasma surrounding the source that contributes significantly to the observed DM. Such dense plasma can cause significant absorption of in the radio regime (see 1.2.3 and Appendix A). The effect of such absorption has been studied in detail (Lyutikov et al., 2016; Piro, 2016).

\subsubsection{Current Results}

A total of 21 FRBs have been published to date but there remain many more questions than answers. Recently, Masui et al. (2015) were able to obtain a rotation measure of an FRB to suggest that most of the contribution to DM comes from the plasma surrounding the source which favors a young magnetar model. Advancements in radio astronomy technology has led to modern telescopes looking for FRBs in real-time, which has increased the number of detections to a great extent. Moreover, future low frequency surveys with telescopes like the MeerKAT (Brederode et al., 2016), the Canadian Hydrogen Intensity Mapping Experiment (CHIME) (Newburgh et al., 2014) and Hydrogen Intensity Realtime Analysis eXperiment (HIRAX) (Newburgh et al., 2016) will increase our sample size by an order of magnitude. CHIME is expected to detect $\sim 100$ FRBs per day, which bodes well for the field in the near future. Chatterjee et al. (2017) have made an 
exciting discovery by localizing the origin of the repeating burst FRB121101. The authors have pin-pointed the source of the FRB to be a dwarf galaxy that is currently forming stars (see Tendulkar et al., 2017, and the references therein). This has posed some interesting questions for progenitors of FRBs and what type of environments are favorable to create them. Based on a precise localization, Marcote et al. (2017) claim that the association of FRB 121102 with the persistent radio source is true and the FRB could be related to a low luminosity AGN or a young supernova remnant powered by a highly energetic neutron star. Though these postulates seem convincing, we need more localizations for FRBs to say anything concrete about their origins.

The localization, along with recent detections of FRBs at the Molonglo radio telescope (Caleb et al., 2017) has shown the potential of interferometers to search for these mysterious bursts. In the near future, the North-South arm of the Molonglo telescope will come online to enhance the localizing capabilities of this already sensitive instrument. With a need for localizations of FRBs, the future is promising for current and upcoming interferometers across the globe.

With latest instruments and technology at our disposal, the future looks bright for pulsar and FRB astrophysics. We can not only discover and study more pulsars and FRBs, but also use these sources to characterize their interaction with ISM/IGM. If we are able to model these interactions, we can potentially use pulsars and FRBs to probe the physics and properties of the intervening medium. The following chapters shows our attempts of studying pulsars, FRBs and using them to gain insights about the ISM. 


\subsection{Outline}

The outline of this dissertation is as follows. In Chapter 2, I discuss our analysis of giga-hertz peaked spectra pulsars using a thermal absorption model. In Chapter 3, I present our results of simultaneous radio and X-ray observations of PSR B0611+22. In Chapter 4, I present results of FRB detections from future low frequency surveys using null results from past surveys. In Chapter 5, I discuss future strategies for detecting pulsars in the Galactic Center. Finally, in Chapter 6 , I present the main conclusions from this work and discuss suggestions for future progress in the field. 


\section{Chapter 2}

\section{On gigahertz spectral turnover in pulsars}

The work presented in this chapter was published as: Kaustubh Rajwade, D. R. Lorimer, Loren Anderson, 'On gigahertz spectral turnovers in pulsars', 2016, MNRAS, Vol. 455, Iss. 1, p. 493

\subsection{Introduction}

Radio flux measurements from pulsars have revealed a wealth of information about the underlying physical processes involved in coherent radio emission (see Graham-Smith, 2003, for a review). A knowledge of the spectral behavior of pulsars is also important for population studies that seek to constrain the luminosity function of the underlying population (see for e.g. Bates et al., 2014). Studies in the past have shown that the flux density, $S$, as a function of frequency, $\nu$, for a pulsar can be described by a simple power law $S \propto \nu^{\alpha}$, with a spectral index $\alpha$ (e.g., Lorimer et al., 1995). Although the observed spectra are found to have a mean value of $\alpha$ around -1.6 (Maron et al., 2000), population models suggest that the underlying population is more consistent with a normal distribution of spectral indices with the mean value around -1.4 (Bates et al., 2013). A small fraction of such sources ( $10 \%$ ) show a broken power-law behavior, with $\alpha$ of $\sim-0.9$ and $\sim-2.2$ (Maron et al., 2000). At low frequencies, synchrotron self absorption becomes dominant in the pul- 
sar magnetosphere and the spectrum tends to show a turnover (Sieber 1973). Such turnovers have been detected for many pulsars around $\sim 100 \mathrm{MHz}$ Sieber (1973).

To date, eleven GPS pulsars have been reported of which two are magnetars (Kijak et al., 2007, 2011; Dembska et al., 2014; Lewandowski et al., 2015). As discussed in 1.2.3, there is correlation between the a turnover and the environment in which the pulsar lies. The strongest argument for environmental origins of highfrequency turnover came from the observations of the binary pulsar B1259-63 (Kijak et al., 2011, hereafter K11a). This pulsar exhibits GPS-like behavior when it is close to its companion Be star LS 2883 and shows a single power-law spectrum when it is far from the Be star. In another study, Kijak et al. (2013) obtained spectra for two magnetars that show GPS-like behavior. Both of the magnetars are associated with supernova remnants (SNRs). The presence of these dense, ionized regions around the pulsars suggests that free-free absorption by the surrounding ionized gas could be responsible for high-frequency turnovers. The authors concluded that pulsars located within ionized environments such as SNRs, H II regions, and PWNe that have high electron densities and emission measures should invariably have high-frequency spectral turnovers.

Motivated by these ideas, a very recent study by Lewandowski et al. (2015) applied a similar model to the one presented here. They show that the absorption is dominant in moderately cold plasma $\left(T_{e} \sim 5000-8000 \mathrm{~K}\right)$ with heightened electron densities (above $\sim 1000 \mathrm{~cm}^{-3} \mathrm{pc}$ ). They use their model to show that the rapid variations in the spectrum of radio magnetar SGR 1745-2900 in the Galactic centre can be explained by free-free thermal absorption of the radio emission by ejecta 
surrounding the magnetar. Using simulations, they were able to show that pulsars can exhibit GPS behavior that can be explained by the model. This is compelling evidence of the dependence of pulsar spectra on their surroundings.

Thermal absorption by free electrons in the vicinity of the GPS pulsars may explain their spectral turnovers as proposed by Kijak et al. (2011) and later by Kijak et al. (2013). Radio emission from pulsars is known to have a steep spectrum that is believed to be due to a non-thermal emission process consisting of pair production in the magnetosphere (see, e.g., Contopoulos \& Spitkovsky, 2006; Melrose \& Yuen, 2014). The characteristics of this emission are similar to those of radio emission observed from SNRs. In this chapter, we modeled the emission from pulsars based on free-free absorption, which has previously been used to explain the radio emission from SNRs. A similar approach was taken by Lewandowski et al. (2015). In contrast to these authors, we have fitted this thermal absorption model to the six known GPS pulsars to constrain the Emission Measure (EM) along the line of sight based on known electron temperatures of the environment surrounding them. In addition, we consider multiple sources of absorbers and try to obtain the most suitable physical conditions for the observed spectrum. We also looked at one bright pulsar that does not show GPS behavior. Using known parameters of EM and electron temperatures for PWNe, we simulated the spectrum of this source to that it can exhibit GPS behavior and discuss about the pulsar's low-frequency turnover. The model, procedure and results of our fitting are explained in section 2. In section 3, we discuss various implications of our results, and in particular highlight the importance of the model on the detectability of pulsars in the Galactic centre. 


\subsection{Model}

\subsubsection{Theory}

Starting from the fundamental equation of radiative transfer (see, e.g., Burke \& Graham-Smith, 2014), we considered 2 scenarios: (i) the pulsar lies within the PWN/SNR or beyond an H II region; (ii) the pulsar lies beyond a cold, partially ionized molecular cloud. In either case, the total measured flux

$$
S_{\mathrm{obs}, \nu}=S_{\mathrm{psr}, \nu} e^{-\tau_{\nu}}+S_{\mathrm{reg}, \nu}\left(1-e^{-\tau}\right)
$$

where $S_{\mathrm{psr}, \nu}$ is the pulsar's intrinsic flux, $S_{\mathrm{reg}, \nu}$ is the flux of the intervening region (PWN or H II region) and $\tau_{\nu}$ is the optical depth at frequency $\nu$ for this line of sight.

We can ignore the $S_{\text {reg, } \nu}$ term because it is a continuum emission that adds to the sky background. Assuming $\tau_{\nu} \sim \nu^{-2.1}$ for free-free absorption and assuming $\tau_{\nu} \ll 1$ for the frequencies of interest (Mezger \& Henderson, 1967) we get

$$
S_{\mathrm{obs}, \nu}=S_{\text {ref }}\left(\frac{\nu}{\nu_{\text {ref }}}\right)^{\alpha} \exp \left[-\tau_{\text {ref }}\left(\frac{\nu}{\nu_{\text {ref }}}\right)^{-2.1}\right]
$$

Here $S_{\text {ref }}$ is some reference flux density measured at a reference frequency $\nu_{\text {ref }}, \alpha$ is the spectral index and $\tau_{\text {ref }}$ is the optical depth at the reference frequency. This is similar to the model previously developed to fit spectra of SNRs (see, e.g., Dulk \& Slee, 1975).

To obtain the optical depth at a given frequency we used the expression given 
in Altenhoff et al. (1960) and Mezger \& Henderson (1967) where we can assume that the medium is optically thin $(\tau \ll 1)$ at high frequencies. Under this assumption, the optical depth

$$
\tau=0.082 a\left(\frac{\nu}{\mathrm{GHz}}\right)^{-2.1}\left(\frac{\mathrm{EM}}{\mathrm{cm}^{-6} \mathrm{pc}}\right)\left(\frac{T_{e}}{\mathrm{~K}}\right)^{-1.35}
$$

where $a$ is a correction factor of the order unity for electron temperatures $T_{e}>20 \mathrm{~K}$ and $\mathrm{EM}$ is the emission measure. Using equations 2.2 and 2.3, we find the spectrum peaks at a frequency

$$
\nu_{\text {peak }}=0.433 \mathrm{GHz}(-\alpha)^{-0.476}\left(\frac{\mathrm{EM}}{\mathrm{cm}^{-6} \mathrm{pc}}\right)^{0.476}\left(\frac{T_{e}}{\mathrm{~K}}\right)^{-0.643}
$$

\subsubsection{Application}

We fitted equation 2.2 to the flux density spectra for six GPS pulsars reported in Kijak et al. (2011). The flux data were taken from Kijak et al. (2007), Kijak et al. (2011) and Dembska et al. (2014). For PSR B1054-62 and PSR J1852-0635, the published errors on the fluxes (Dembska et al., 2014) were substantially smaller than the errors for other pulsars, which were around 20\%. We therefore increased the errors on these two pulsars to make them comparable with the rest of the sample. We did this fitting for two scenarios: one for warm plasma with characteristic properties of H II regions or PWNe and one for cold, partially ionized clouds.

For the first scenario, we use characteristic properties of PWNe/H II regions, which are known to have $T_{e} \simeq 5000-10^{4} \mathrm{~K}$ for $\mathrm{H}$ II regions and $T_{e} \simeq 10^{4}-10^{6} \mathrm{~K}$ for 
PWNe (e.g., Slane et al. 2004). The value of $T_{e}$ for the PWN/H II region scenario was fixed to $5000 \mathrm{~K}$ as Lewandowski et al. (2015) show that the absorption of radio emission takes place in plasma with ionized gas filaments with $T_{e} \sim 5000 \mathrm{~K}$ in SNRs (Sankrit et al., 1998; Koo et al., 2007). Such high density plasma also exists in young "ultra compact" star forming H II regions where electron densities are of the order of $\sim 10^{4} \mathrm{~cm}^{-3}$ (for a review, see Churchwell, 2002) so it is also possible that a pulsar lying beyond an H II region might experience absorption of radio emission. Pulsars within PWNe may not exhibit GPS spectra because the PWNe plasma distribution is inhomogeneous and there may not be a dense filament between us and the pulsar.

For the second scenario, we use characteristic properties of cold, partially ionized clouds, which Dulk \& Slee (1975) suggest are the most promising absorbers of radio emission from SNRs. For such clouds, we used $T_{e}=30 \mathrm{~K}$. Due to asymmetries in the PWN shell, the radio emission may be absorbed by the cold clouds instead of the filaments. However, PWN are often found in the vicinity of molecular clouds with a high rate of star formation. We kept $\alpha$ and $\tau$ as free parameters as we believe that there could be a bias in the measured values of $\alpha$ due to the high-frequency turnover behavior. The value of EM can be calculated from the derived value of $\tau$ and by assuming a $T_{e}$. For this case, we assumed $30 \%$ uncertainties in the value of $T_{e}$, and propagated this uncertainty to derive uncertainties in EM. The value of $30 \%$ is arbitrary in the sense that it only affects the errors on derived values of EM and not the values themselves. We select $S_{\text {ref }}$ and the corresponding $\tau_{\text {ref }}$ from the highest frequency measurement since the effects of free-free absorption should be negligible 
at those frequencies and therefore the assumptions we made for $\tau_{\text {ref }}$ would hold. Using this as our starting point, we fitted the model to the observed spectra for the two different electron temperatures scenarios mentioned above using the LevenbergMarquardt non-linear least squares algorithm (see, e.g., Press et al. 2010) and for each pulsar derived the values for EM and $\alpha$ given in Table 2.1.

The values of EM we derived in Table 2.1 have rarely been measured before. Here, we give a possible physical explanation of why such high values can arise. From the simulations done in Lewandowski et al. (2015), one infers that to observe GPS behavior, a pulsar needs to be beyond a region of ionized gas a fraction of a parsec thick with ambient temperatures of the order of a few $1000 \mathrm{~K}$ and relatively high free electron density. High electron densities of $\left(\sim 2000-6000 \mathrm{~cm}^{-3}\right)$ with relatively cooler temperatures $(\sim 5000-8000 \mathrm{~K})$ have been found to exist in dense filaments a fraction of a parsec wide around SNRs and PWNs (Sankrit et al., 1998; Koo et al., 2007). For example, if we consider the line of sight along the filament found by Koo et al. in SNR G11.2-0.3, we can infer an EM contribution due to filament by using the values in their paper which is $\sim 10^{7} \mathrm{~cm}^{-6} \mathrm{pc}$. This is of the same order as our derived values. If we assume that the derived EM contributes to the total DM by a fraction $a$ then the linear size of the filament

$$
d_{\text {filament }}=a^{2}\left(\frac{\mathrm{DM}}{\mathrm{cm}^{-3} \mathrm{pc}}\right)^{2}\left(\frac{\mathrm{EM}}{\mathrm{cm}^{-6} \mathrm{pc}}\right)^{-1} \mathrm{pc}
$$

The values obtained from Equation 2.5 reflect the dimension of the filament along 
the line of sight. For the absorbing medium, the mean electron density

$$
\left\langle n_{e}\right\rangle=\frac{1}{a}\left(\frac{\mathrm{DM}}{\mathrm{cm}^{-3} \mathrm{pc}}\right)^{-1}\left(\frac{\mathrm{EM}}{\mathrm{cm}^{-6} \mathrm{pc}}\right) \mathrm{cm}^{-3}
$$

We estimate the electron density and the linear size of the absorber by assuming a DM fraction of $50 \%$ for the GPS sources in this chapter. We did the analysis for the absorption scenarios considered for the previous analysis. From our results, we infer the most likely source of absorption for all the pulsars in our sample. The values of electron density, linear size and the source of absorption are listed in Table 2.2. The values we obtained for $\nu_{\text {peak }}$ differ from values given K11 simply because K11 calculate the turnover frequency from the intersection of two linear fits to the spectrum. To quantify the quality of the fit even further, we obtained the reduced $\chi^{2}$ by fitting the pulsar fluxes with a single power law and compared them to the ones obtained by the model. The values for the power law are higher by at least a factor of two, suggesting that the model given in equation 2.2 is preferred over the power-law model.

The model fits the observed data well, as illustrated by Fig. 2.2 and the reduced $\chi^{2}$ values given in Table 2.1. This motivated us to look at pulsars that do not lie in dense environments and to simulate their spectra to examine if the low-frequency turnover is due to the environment or the pulsar itself. For this purpose, we selected one bright, non-GPS pulsar, PSR B0329+54, for which there are reliable estimates of flux densities at different frequencies and spectral index in literature (Kramer et al., 2003). Using the model, we tried to fit the spectrum of this pulsar. The 


\begin{tabular}{lccclclc}
\hline PSR & $\alpha$ & $\tau$ & $\begin{array}{c}\mathrm{EM} \\
\left(10^{6} \mathrm{pc} \mathrm{cm}^{-6}\right)\end{array}$ & & $\begin{array}{c}\nu_{\text {peak }} \\
(\mathrm{GHz})\end{array}$ & $\begin{array}{l}\chi^{2} \\
\text { Model }\end{array}$ & $\begin{array}{c}\chi^{2} \\
\text { Power law }\end{array}$ \\
\hline & & & $5000 \mathrm{~K}$ & $30 \mathrm{~K}$ & & & \\
\hline B1054-62 & $-2.8(8)$ & $0.19(3)$ & $1.1(9)$ & $0.0006(4)$ & 0.6 & 3.5 & 6.5 \\
J1809-1917 & $-2.5(4)$ & $0.16(3)$ & $5.2(3)$ & $0.005(3)$ & 1.8 & 1.2 & 7.3 \\
B1822-14 & $-0.6(1)$ & $0.005(1)$ & $0.2(1)$ & $0.0002(1)$ & 0.7 & 1.7 & 16.8 \\
B1823-13 & $-0.8(1)$ & $0.003(1)$ & $0.5(2)$ & $0.0005(2)$ & 1.0 & 2.3 & 46.5 \\
J1740+1000 & $-2.0(1)$ & $0.007(3)$ & $0.8(1)$ & $0.0008(1)$ & 0.8 & 12 & 122.8 \\
J1852-0635 & $-1.1(1)$ & $0.006(2)$ & $0.7(3)$ & $0.0007(3)$ & 1.0 & 1.3 & 3.8 \\
\hline
\end{tabular}

Table 2.1: For each pulsar, we list the derived values of $\alpha$ and $\tau$ from fitting Equation 2 to the spectra. Also listed are the assumed electron temperature and derived EM using Equation 3, $\nu_{\text {peak }}$ calculated from Equation 4 as well as the reduced $\chi^{2}$ values from fitting out model versus a simple power law. Figures in parentheses represent the formal uncertainties in the least significant digits.

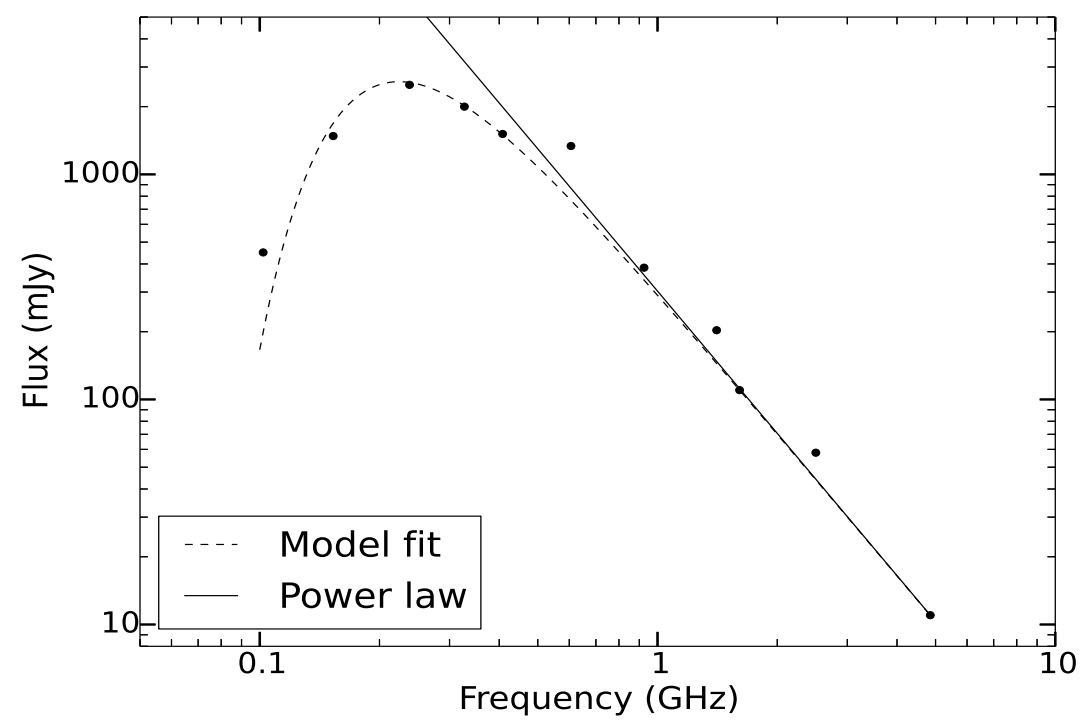

Figure 2.1: Power law spectrum (solid line) and the best fit curve (dashed line) from the model for PSR B0329+54. The black filled circles are measured flux densities taken from literature. The power law has a spectral index $\alpha=-2.1$ taken from the literature. The model fit has a reduced $\chi^{2}$ value of 1.8 . 

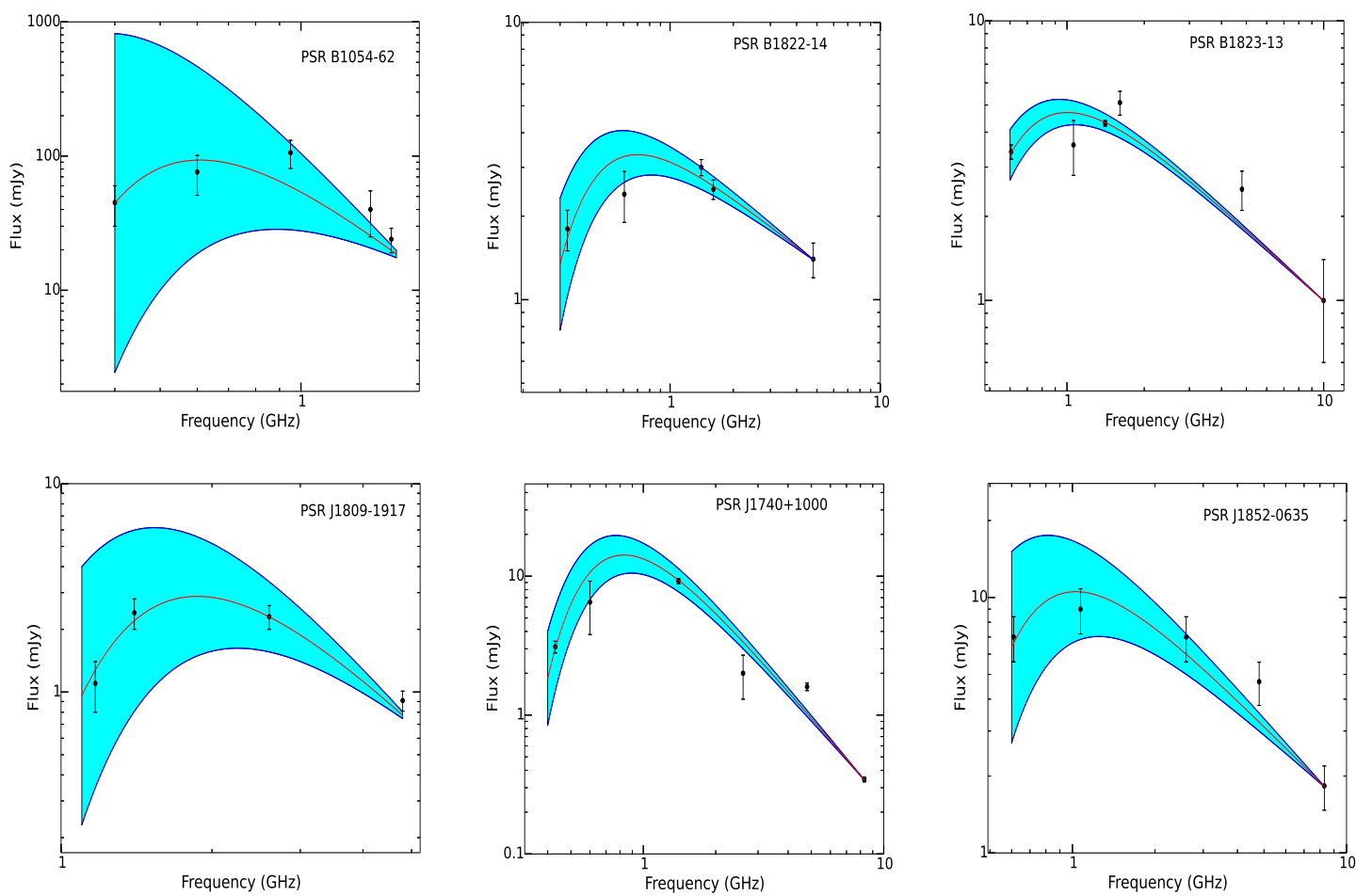

Figure 2.2: Best fit curve along with $\pm 1 \sigma$ region (shaded) obtained from the thermal absorption model for the set of GPS pulsars. Black circles are measured flux densities taken from Kijak et al. (2007), Kijak et al. (2011) \& Dembska et al. (2014). The $\pm 1 \sigma$ shaded region is determined by assuming a Gaussian error on the derived parameters and using $\pm 1 \sigma$ limits of these parameters to obtain the curves.

\begin{tabular}{llllll}
\hline PSR & \multicolumn{2}{l}{$\begin{array}{l}\text { Electron density } \\
\left(\mathrm{cm}^{-3}\right)\end{array}$} & $\begin{array}{l}\text { Linear size } \\
(\mathrm{pc})\end{array}$ & Absorber \\
\hline & $5000 \mathrm{~K}$ & $30 \mathrm{~K}$ & $5000 \mathrm{~K}$ & $30 \mathrm{~K}$ & \\
\hline B1054-62 & $3.6 \times 10^{3}$ & 3.6 & 0.044 & 48 & Cold, partially ionized cloud \\
$\mathrm{J} 1809-1917$ & $5.3 \times 10^{4}$ & 53.5 & 0.002 & 1.8 & Cold, partially ionized cloud \\
B1822-14 & $0.9 \times 10^{3}$ & 0.9 & 0.2 & 195 & Dense, ionized filament \\
B1823-13 & $4.0 \times 10^{3}$ & 4.2 & 0.02 & 27.2 & Dense, ionized filament \\
$\mathrm{J} 1740+1000$ & $6.6 \times 10^{4}$ & 66 & 0.0002 & 0.2 & Cold, partially ionized cloud \\
$\mathrm{J} 1852-0635$ & $8.0 \times 10^{3}$ & 7.7 & 0.01 & 11 & Cold, partially ionized cloud \\
\hline
\end{tabular}

Table 2.2: Constrained parameters for all GPS pulsars in our sample by assuming an absorber contribution of $50 \%$ to the total DM of the pulsar. To infer the absorber, we assumed $n_{e}=100-6000 \mathrm{~cm}^{-3}$ for a dense, ionized filament and $n_{e}=1-100 \mathrm{~cm}^{-3}$ for a cold, partially ionized cloud and also considered the known environment around the pulsar. PSR B1054-62 was an exception (see text for details). 
slight turnover seen in the actual data for PSR B0329+54 is at $\sim 200 \mathrm{MHz}$. We believe that this turnover is intrinsic to the pulsar itself. Hence, the fit should give us values of EM due to ISM, or it may give some unreasonable values which might suggest a different mechanism for the low-frequency turnover seen in Fig. 2.1. To show this, we fitted for the EM of PSR B0329+54 by constraining the electron temperature, which we assume to be $5000 \mathrm{~K}$ (i.e., that of the Warm Ionized Medium (WIM) (Madsen et al., 2006)). The value we obtained was $5.2 \times 10^{4} \mathrm{~cm}^{-6}$ pc. If we assume a WIM-dominated ISM between the pulsar and the observer, we can use the measured parallax for PSR B0329+54 (Brisken et al., 2002) and its measured dispersion measure (DM), the integrated electron density along the line of sight to find a mean electron density of $\sim 0.024 \mathrm{~cm}^{-3}$. Assuming a filling factor of 0.1 (Berkhuijsen \& Müller, 2008) to account for the clumpiness of the ISM, and knowing the distance to the pulsar, we derive a value of EM of $\sim 0.052 \mathrm{~cm}^{-6} \mathrm{pc}$. This value of $\mathrm{EM}$ is $\sim 6$ orders of magnitude smaller than the one derived from the model which suggests that the turnover in spectrum of PSR B0329+54 could be due to synchrotron self-absorption in the pulsar's magnetosphere. Also, if we fix the $\mathrm{EM}$ to $\sim 0.052 \mathrm{~cm}^{-6} \mathrm{pc}$ and fit for the electron temperature, the value we obtain is $0.18 \mathrm{~K}$ which is nonsensical for a WIM-dominated ISM.

\subsection{Discussion}

Long-period pulsars are known to show turnovers in their flux density spectra at frequencies of $\sim 100 \mathrm{MHz}$ (Maron et al. 2000). It is proposed that at such low 
frequencies, the radio emission becomes optically thick because of synchrotron selfabsorption (O'Dea, 1998; Chevalier, 1998). From the thermal absorption model, it is seen that if the pulsar were to lie in an extremely dense environment, free-free absorption in the dense region can dominate at frequencies higher than $100 \mathrm{MHz}$ depending on the electron density and electron temperature of the environment. This results in the pulsar flux being absorbed by the surrounding material, which manifests itself as a high-frequency turnover. This study is consistent with the claim that GPS behavior does not depend on the DM of the pulsar (Kijak et al. 2011; Dembska et al. 2014). All pulsars lying in a high electron density environment would invariably have high DMs but we measure a higher DM even if the pulsar is further away from us and not necessarily in a dense environment. If this were not true, all pulsars with a high DM would have shown GPS-like characteristics. It is important to note that only pulsars where the line of sight traverses through such dense filaments might show GPS behavior. We derive large values for the EM for which we consider two physical scenarios based on previous studies (Dulk \& Slee, 1975; Lewandowski et al. 2015). Either the pulsar flux is absorbed by the high density, ionized filaments surrounding the PWNe/SNRs or by the cold, partially ionized clouds along the line of sight. Using this idea, and the fact that the absorbers only contribute to a part of the observed DM, we calculate the mean electron density of the absorbers. Assuming a certain range of values of $n_{e}$ for each scenario $\left(n_{e} \sim 100-6000 \mathrm{~cm}^{-3}\right.$ for SNR filaments and $n_{e} \sim 1-100 \mathrm{~cm}^{-3}$ for cold molecular clouds) and considering the known environment of each pulsar, we report the most plausible absorber for each pulsar in our sample in Table 2.2. For PSR B1054-62, both the scenarios give 
reasonable estimates for the electron densities. We believe that a cold absorber is more suitable because, although the pulsar lies near an H II region RCW55 (Koribalski et al. 1995), it lies at the very edge of the region so there is not enough high density, ionized material to produce the observed spectrum. Also, the fact that PSR B1054-62 lies in the Carina complex, a large molecular complex with high star formation, strengthens our claim. The calculations of electron density provides an independent estimate of electron densities within the dense clumps of the ISM that can be very difficult to obtain by conventional methods as most of emission we detect from these sources is non-thermal synchrotron emission (Kargaltsev et al., 2007).

The model also can be useful to gain insights into the emission physics of millisecond pulsars. Investigations in the past have not shown any trend of a turnover in millisecond pulsar spectra (Kramer et al., 1999). In recent years, however, with the advent of high sensitivity data acquisition systems, these pulsars are routinely detectable over a wide range of radio frequencies. Recent work by $\mathrm{Ku}-$ niyoshi et al. (2015) shows that a number of millisecond pulsars are likely to have spectral turnovers at frequencies in the range $0.5-1 \mathrm{GHz}$. A future application of this model will be to investigate whether any of these pulsars lie within dense environments and use the model to probe the ISM in the vicinity of the pulsar.

The size and characteristics of the pulsar population in the Galactic centre (GC) has been a puzzle for astronomers for the past few years. Several authors have tried to constrain the population of pulsars in the GC using various techniques (see, e.g., Chennamangalam \& Lorimer 2014; Macquart \& Kanekar 2015) that try 


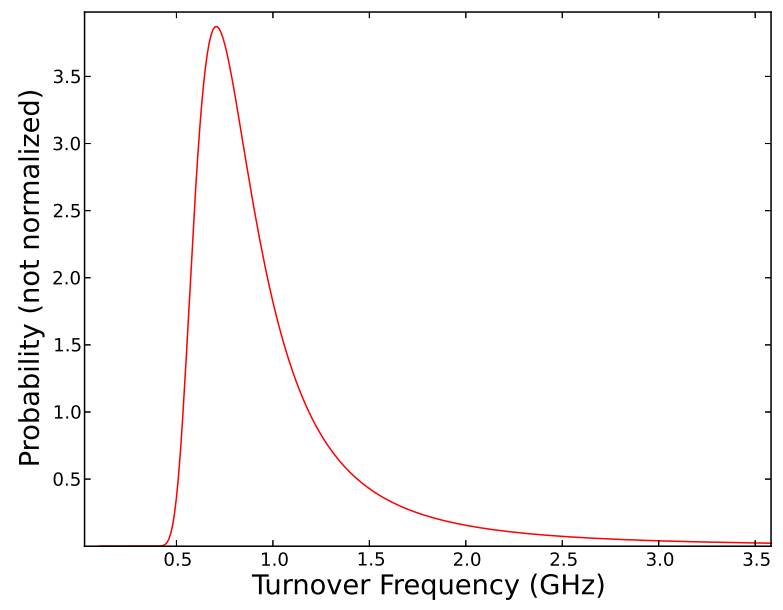

$\mathrm{O}$

Figure 2.3: Sample expected probability density function of turnover frequencies for a putative line-of-sight to the Galactic center obtained using Equation 4 and assuming a distribution of spectral indices for a sample population of pulsars in the Galactic center (see text).

to account for a number of observational selection effects. The model discussed here might have potential implications on such work. If we adopt values from the literature for model parameters for a line of sight to the $\mathrm{GC}, \mathrm{EM}=5 \times 10^{5} \mathrm{~cm}^{-6} \mathrm{pc}$ and $T_{e}=5000 \mathrm{~K}$ (see, e.g., Pedlar et al., 1989), and take a distribution of intrinsic power-law spectral indices with mean -1.4 and unit standard deviation (Bates et al., 2014), using equation 2.4, it is straightforward to show that there is a distribution of turnover frequencies that extends down to a GHz (see Fig. 2.3). This distribution suggests that approximately half of all GC pulsars might exhibit spectral turnovers at frequencies greater than $1 \mathrm{GHz}$. Such pulsars would be harder to detect than previously thought. Recently, there have been targeted pulsar surveys of the GC at frequencies higher than $1 \mathrm{GHz}$ (see, e.g. Macquart et al., 2010; Deneva, 2010) that should not be greatly affected by the spectral turnovers. The absence of of 
any detected pulsars in these surveys led Chennamangalam \& Lorimer (2014) to conclude that there are very few of these sources in the GC. The results found here suggest that the detectability of pulsars in the GC region may be impacted by spectral turnovers due to the dense environment. We plan to quantify the impacts of this issue on GC pulsar population size constraints further in a future paper.

In summary, we have presented an application of a simple free-free absorption model, also proposed by Lewandowski et al. (2015), which is consistent with the turnover in the spectra of GPS pulsars being caused by propagation through a dense medium. The results of the thermal absorption model strengthen the claim that high-frequency spectral turnovers have their origins in the medium surrounding the neutron star. We were able to determine the most sensible source of absorption for each pulsar using an estimate for the mean electron density within the cloud. More refined measurements of pulsar fluxes, and more examples of GPS pulsars, are essential to test the model further. 


\section{Chapter 3}

\section{Simultaneous radio and X-ray observations of PSR B0611+22}

The work presented in this chapter was published as: Kaustubh Rajwade, Andrew Seymour, D. R. Lorimer, Aris Karastergiou, Maciej Serylak, M. A. McLaughlin, J-M Griessmeier, 'Simultaneous Radio and X-ray observations of PSR B0611+22', 2016, MNRAS, Vol. 462, Iss. 3, p 2518

\subsection{Introduction}

The 0.33 s pulsar PSR B0611+22 (characteristic age 90 kyr) was discovered by Davies et al. (1972) and was initially thought to be associated with the supernova remnant (SNR) IC 443 which lies at close angular separation to the pulsar (Davies et al., 1972; Hill, 1972). This association was always doubtful as the pulsar lies well beyond the radio shell (Duin \& van der Laan, 1975) of the remnant. Recent X-ray observations detected a compact X-ray source within the remnant shell and the corresponding pulsar wind nebula (Olbert et al., 2001) which rejected any association of the pulsar with the remnant. Moreover, IC 443 is known to lie within the molecular cloud G189+3.3 (Bocchino \& Bykov, 2000) which lies along the line of sight to the pulsar. Although, the distances to these sources are highly uncertain, it is reasonable to assume that the pulsar lies beyond these dense regions (Fesen, 1984; Welsh \& Sallmen, 2003). This suggests that the radio emission propagates through 
the dense medium which might contribute to the pulsar's dispersion measure (DM) of $\sim 96 \mathrm{pc} \mathrm{cm}^{-3}$. The environment of this pulsar makes it an interesting object for studies of radio emission and single-pulse properties.

The pulsar was studied by Nowakowski (1992), who found that PSR B0611+22 appears to exhibit different modes in which the enhanced emission mode peaked at a later pulse phase than the average profile and the weak mode peaked at an earlier phase. Recently, Seymour et al. (2014) performed a detailed study of the emission behavior of PSR B0611+22. They found that, at $327 \mathrm{MHz}$, the pulsar shows steady emission in one mode which is enhanced by bursting emission that is slightly offset in pulse phase from this steady emission. Seymour et al. (2014) also observed the bursting to be quasi-periodic with a period around $\sim 1000$ pulse periods. This type of behavior has also been seen in other pulsars like PSR J1752+2358 (Gajjar et al., 2014) and PSR J1938+2213 (Lorimer et al., 2013). PSR B0611+22's short mode changes with offset in the emission phase could be responsible for the high degree of timing noise the pulsar exhibits (Arzoumanian et al., 1994).

The phenomena of nulling and mode changing which relate to such emission behavior have been studied in different pulsars for four decades. They were first observed and reported by Backer (Backer, 1970a,b,c,d). Mode changing pulsars are pulsars in which, from time to time, the mean profile abruptly changes between two or more quasi-stable states (Wang et al., 2007; Bartel, 1982) while nulling is the abrupt cessation of radio emission for one or more pulse periods. Nulling has been postulated to be an extreme case of mode changing (Wang et al., 2007; Timokhin, 2010). In a series of papers, Rankin (Rankin, 1983, 1986; Rankin \& Ramachan- 
dran, 2003) tried to understand the emission geometry and behavior of such pulsars. According to her model, the emission beam of a pulsar consists of a central core emission beam surrounded by multiple annular cones of emission. The pulse profile we observe depends on which core and/or cone beams are traversed by the line of sight of the observer. Rankin suggested that mode changing can be thought of as a reorganization of such core and conal emission resulting in a change in the observed pulse profile. Mode changing has been observed in most multi-component pulsars (pulsars with more than one component in their emission profile) (Rankin, 1986). Many pulsars like PSR B2319+60 (Wright \& Fowler, 1981), PSR B0943+10 (Suleymanova et al., 1998) and PSR B1918+19 (Rankin et al., 2013) exhibit this phenomenon. Both nulling and mode changing have been studied in $~ 200$ pulsars so far (Biggs, 1992; Weltevrede et al., 2006; Wang et al., 2007; Gajjar et al., 2012). PSR B0611+22 has been classified as a core emission pulsar with a single component (Rankin, 1983). This makes the pulsar interesting as the phase offsets and flux enhancement are small in comparison to other pulsars in terms of magnitude and timescale and are harder to explain in the standard framework. Recently, a global picture of quasi-stable states of the magnetosphere has come to the fore (Lyne et al., 2010; Hermsen et al., 2013).

Hermsen et al. (2013) discovered an anti-correlation between X-ray and radio emission in the two modes of emission of PSR B0943+10. This result motivated us to ask whether such X-ray emission is also detectable in PSR B0611+22 and, if yes, how does it relate to the mode changes seen in radio? This led to a simultaneous radio and X-ray observation campaign of PSR B0611+22. As mentioned above, 
PSR B0611+22 has a supernova remnant and a molecular cloud in its vicinity. Such dense environments around and likely, in front of the pulsar make it an ideal candidate to study the effects of these environments on the measured flux density. Previously, pulsars within such dense environments have been known to show a spectral turnover at frequencies around $\sim 1 \mathrm{GHz}$ (Kijak et al., 2007, 2011). A recent study by Rajwade et al. (2016a) shows that it is possible to derive the physical parameters of these dense regions by modeling the flux density spectrum of the pulsar. In this chapter, we try to characterize the peculiar emission behavior with a multi-wavelength, broadband dataset of the pulsar. The observational details are given in Section 2. The results are presented in Section 3. The discussions based on the results are in Section 4. The conclusions are given in Section 5.

\subsection{Observations}

All observations were carried out on MJD 56756. PSR B0611+22 was observed at three different radio frequencies including $327 \mathrm{MHz}$ (Arecibo Observatory), $820 \mathrm{MHz}$ (Green Bank Telescope) and $150 \mathrm{MHz}$ (International LOFAR stationNançay, France). The observation configurations for the radio telescopes are given in Table 3.1. The data were recorded and converted into multi-channel filterbank format before being written out to disk. Then, the data were incoherently dedispersed using PRESTO ${ }^{1}$ at the pulsar DM of $96 \mathrm{pc} \mathrm{cm}^{-3}$ to remove the dispersion delay of incoming radio waves due to the interstellar medium. For the LOFAR

\footnotetext{
${ }^{1}$ http://www.cv.nrao.edu/ sransom/presto
} 
(FR606) data, coherent dedispersion was carried out using DSPSR ${ }^{2}$ (van Straten et al., 2011).

Simultaneously, the pulsar was observed by XMM-Newton. The XMM-Newton observations used the photon imaging camera (EPIC) (Strüder et al., 2001; Turner et al., 2001). The PN-CCD was operated in small-window mode with a medium filter to block stray optical light. All the events recorded by the PN camera are timetagged with a temporal resolution of $5.7 \mathrm{~ms}$. On the other hand, the MOS CCDs were operated in full-window mode with a medium filter in each camera, which provide us with a large field-of-view. The coverage of various telescopes during the whole observation is illustrated in Table. 3.1.

\footnotetext{
${ }^{2}$ http://dspsr.sourceforge.net
} 


\begin{tabular}{lcccccccl}
\hline Telescope & $\begin{array}{c}\nu \\
(\mathrm{MHz})\end{array}$ & $\begin{array}{c}t_{\text {samp }} \\
(\mathrm{ms})\end{array}$ & $\begin{array}{c}\Delta \nu \\
(\mathrm{MHz})\end{array}$ & $\begin{array}{l}t_{\text {int }} \\
(\mathrm{hrs})\end{array}$ & $n_{\text {chan }}$ & $\begin{array}{l}G \\
(\mathrm{~K} / \mathrm{Jy})\end{array}$ & $\begin{array}{l}T_{\text {sys }} \\
(\mathrm{K})\end{array}$ & $\begin{array}{l}\mathrm{UT} \\
\mathrm{hh}: \mathrm{mm}\end{array}$ \\
\hline Green Bank Telescope (GBT) & 820 & 0.15 & 200 & 1.25 & 2048 & 2 & 101 & $16: 30-17: 45$ \\
Arecibo Observatory (AO) & 327 & 0.5 & 50 & 2 & 2048 & 11 & 117 & $20: 30-22: 30$ \\
LOFAR (FR606) & 148 & 0.32 & 80 & 6 & 400 & 0.97 & 900 & $16: 45-22: 30$ \\
XMM-Newton & & & & & & & & $16: 30-22: 30$ \\
\hline
\end{tabular}

Table 3.1: Configuration of different radio telescopes during observation of PSR B0611+22. From left to right, we list the observing frequency $(\nu)$, sampling time $\left(t_{\text {samp }}\right)$, bandwidth $(\Delta \nu)$, observation time $\left(t_{\text {int }}\right)$, number of frequency channels $\left(n_{\text {chan }}\right)$ gain $(G)$, system temperature $\left(T_{\text {sys }}\right)$ and Universal Time (UT) of observation. 


\subsection{Results}

\subsubsection{Broadband bursting}

We analyzed the radio data for bursting behavior at different frequencies similar to Seymour et al. (2014). For each frequency, namely 820, 327 and $150 \mathrm{MHz}$, the time series were folded at the topocentric period of the pulsar to generate the averaged pulse profiles shown in Fig. 3.1.

For these folded time series (see Fig. 3.5), we Fourier transformed the energies at each pulse longitude (phase bin) to obtain a longitude resolved fluctuation spectrum for 327 and $820 \mathrm{MHz}$. The fluctuation spectrum was integrated over specific On and Off pulse windows to obtain the integrated power spectrum for both regions as shown in Fig. 3.2. The figure suggests that bursts seem to be quasi-periodic at both frequencies. We derived a rough periodicity for the bursts of $\sim 2500$ pulse periods from Fig. 3.2. The periodic nature of emission is evident in Fig. 3.3 which shows how energy of a single bin corresponding to the peak in the average profile varies in time. The profile was chosen from the ON pulse window at 327 and 820 $\mathrm{MHz}$ as done in Seymour et al. (2014).

Due to telescope scheduling constraints, there was no overlap between the 327 and $820 \mathrm{MHz}$ observations. However, each of those observations overlapped with the $150 \mathrm{MHz}$ observations so we decided to compare the observations at $327 \mathrm{MHz}$ and $820 \mathrm{MHz}$ with the corresponding spans in the $150 \mathrm{MHz}$ observations. Since we do not have high enough sensitivity from the LOFAR (FR606) observations to detect single pulses, we decided to convolve the pulse stack using a 2-D Gaussian kernel 


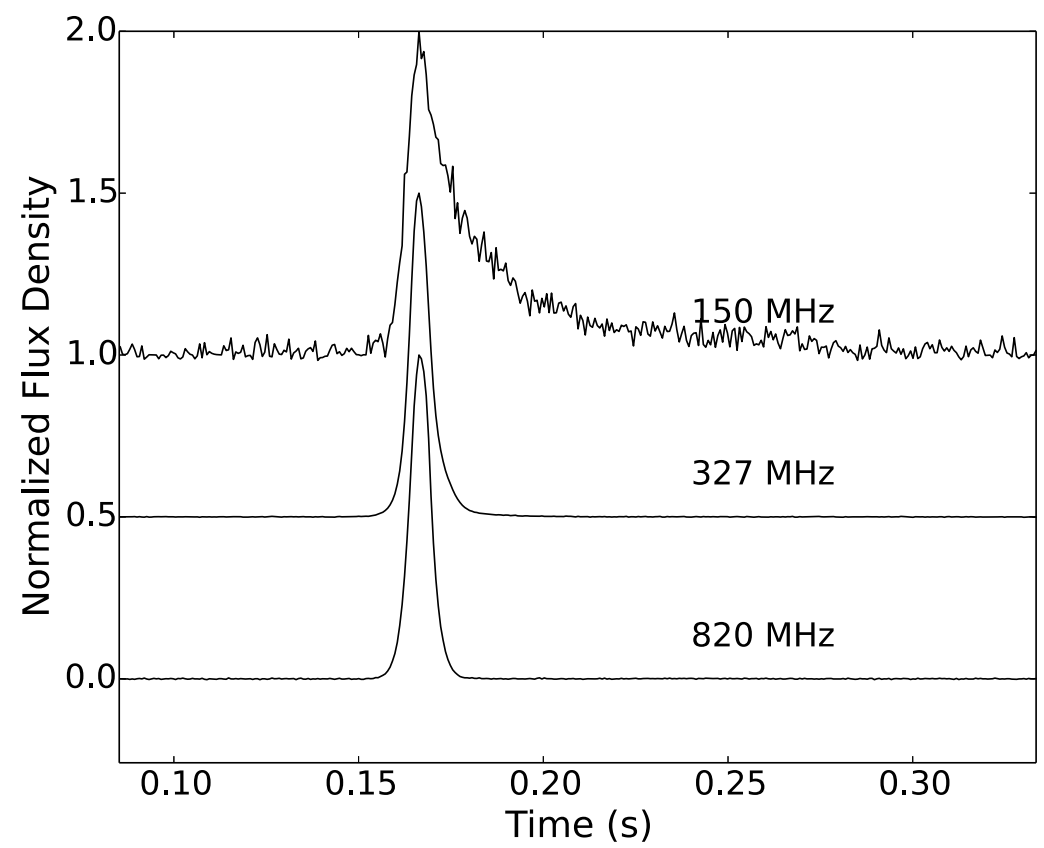

Figure 3.1: The peak of the average profile of PSR B0611+22 at different radio frequencies. For clarity, only part of the period near the peak is plotted.

with a width spanning 64 time bins (i.e. single pulses) and 5 pulse phase bins. We applied the same smoothing to each dataset to give them comparable resolution. To ensure time alignment, we removed dispersion delay for each telescope using infinite frequency as the reference. The timeseries for each dataset were barycentered to remove any time delays due to different locations of the telescopes.

By doing this, we were able to obtain snippets of datasets where we could study the bursting behavior simultaneously at different radio frequencies. Figure 3.5 clearly shows enhanced emission to study broadband correlations. The emission exhibits a change in its behavior going from 820/150 MHz observations to $327 / 150 \mathrm{MHz}$ observations. The direction of phase shift during bursting is different at 327 and $820 \mathrm{MHz}$ when compared to their corresponding $150 \mathrm{MHz}$ datasets. 

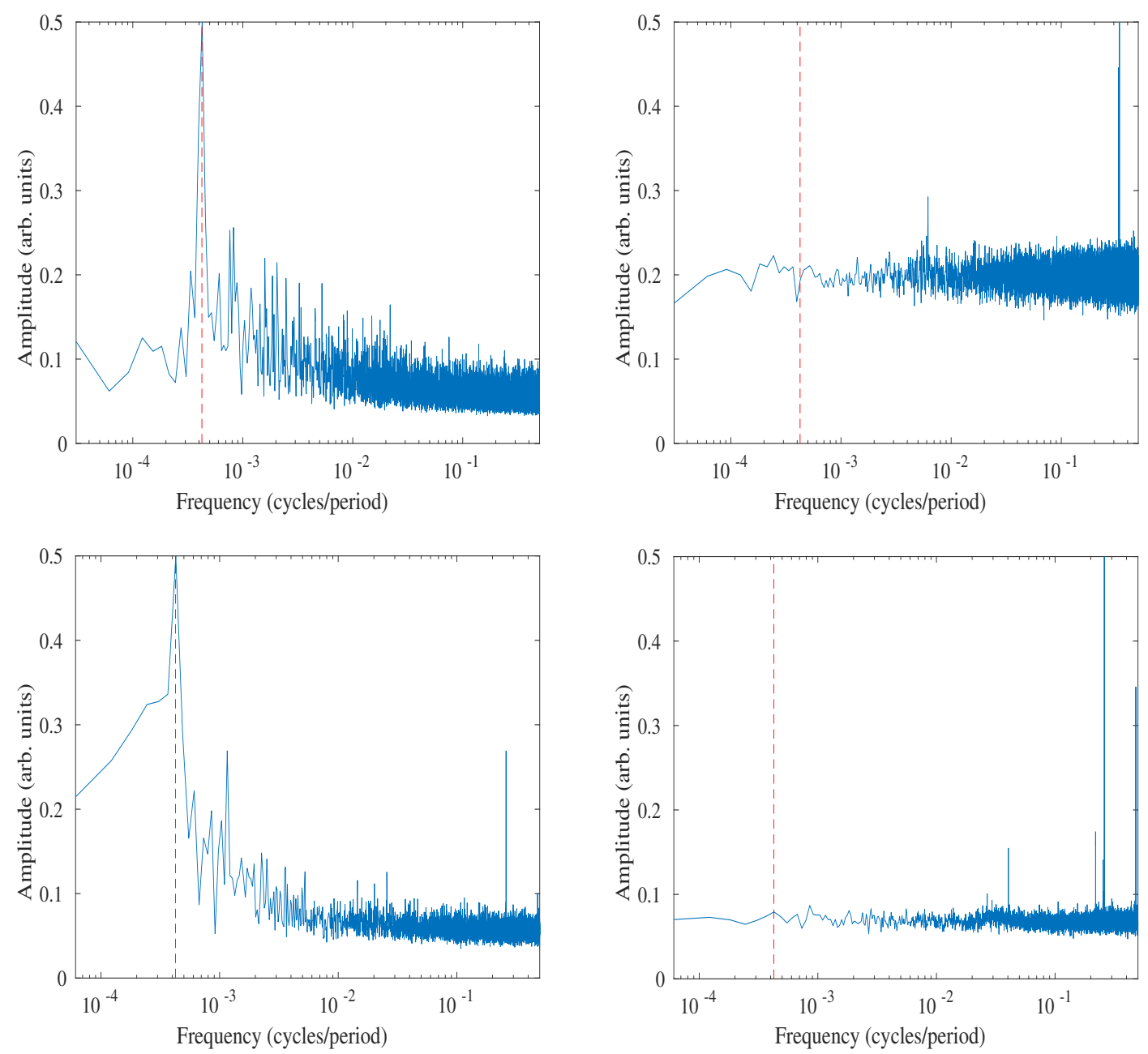

Figure 3.2: Fluctuation spectrum for PSR B0611+22 for Top Panels: $327 \mathrm{MHz}$ and Bottom Panels: $820 \mathrm{MHz}$. The left panels show the integrated On pulse power spectrum and the right panels show the integrated Off pulse power spectrum. The red vertical line corresponds to a frequency of $\sim 0.0004$ cycles/period. One can see the spike at $\sim 0.0004$ cycles/period in the top panels corresponding to a period of 2500 pulse periods at both frequencies. 

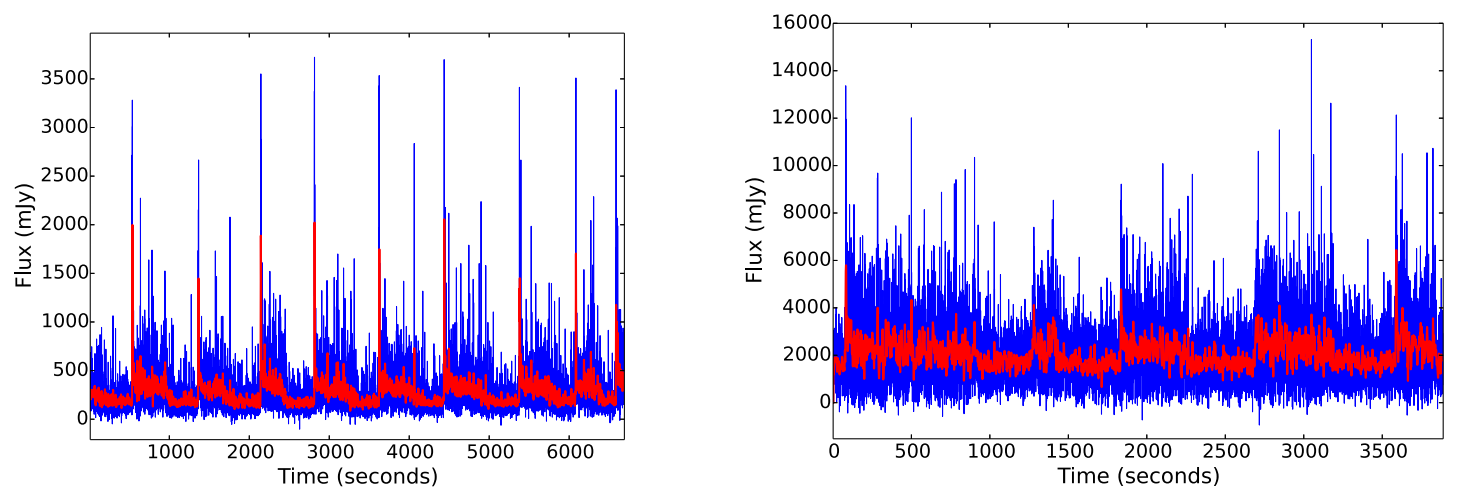

Figure 3.3: Flux time series of a single bin of PSR B0611+22 Left Panel: $327 \mathrm{MHz}$ (duration $\simeq 2$ hours) and Right Panel: $820 \mathrm{MHz}$ (duration $\simeq 1.25$ hours). The blue line is the full resolution data. The red line is the smoothed version.

The correlation in $327 / 150 \mathrm{MHz}$ and the anti-correlation in $820 / 150 \mathrm{MHz}$ of the bursting is evident in Fig. 3.4 which shows the cross-correlations between the two frequencies. Fig. 3.4 reveals a slight offset in the maximum of the $327 / 150 \mathrm{MHz}(\sim 60$ bins) and the minimum of the $820 / 150 \mathrm{MHz}$ ( 50 bins) plot from the zero lag. Since the offsets are comparable to the kernel width used to smooth the datasets along the time axis, they are insignificant and most likely not intrinsic to this phenomena.

We divided the time series into sections with no bursting and where bursting was clearly evident. The sections were selected visually from the smoothed data. From these sections, average profiles were created by summing the energies over the phase bins where the pulsed emission was seen. The profiles clearly bring out the difference in the behavior of bursting at both frequencies. The profile for the bursting pulses is phase shifted in pulse longitude at the two frequencies as reported by Seymour et al. (2014) although there is a difference in the direction of the phase shift. At $327 \mathrm{MHz}$, the phase shift occurs towards the trailing edge of the profile while it occurs at the leading edge of the profile at $820 \mathrm{MHz}$. This is illustrated in 

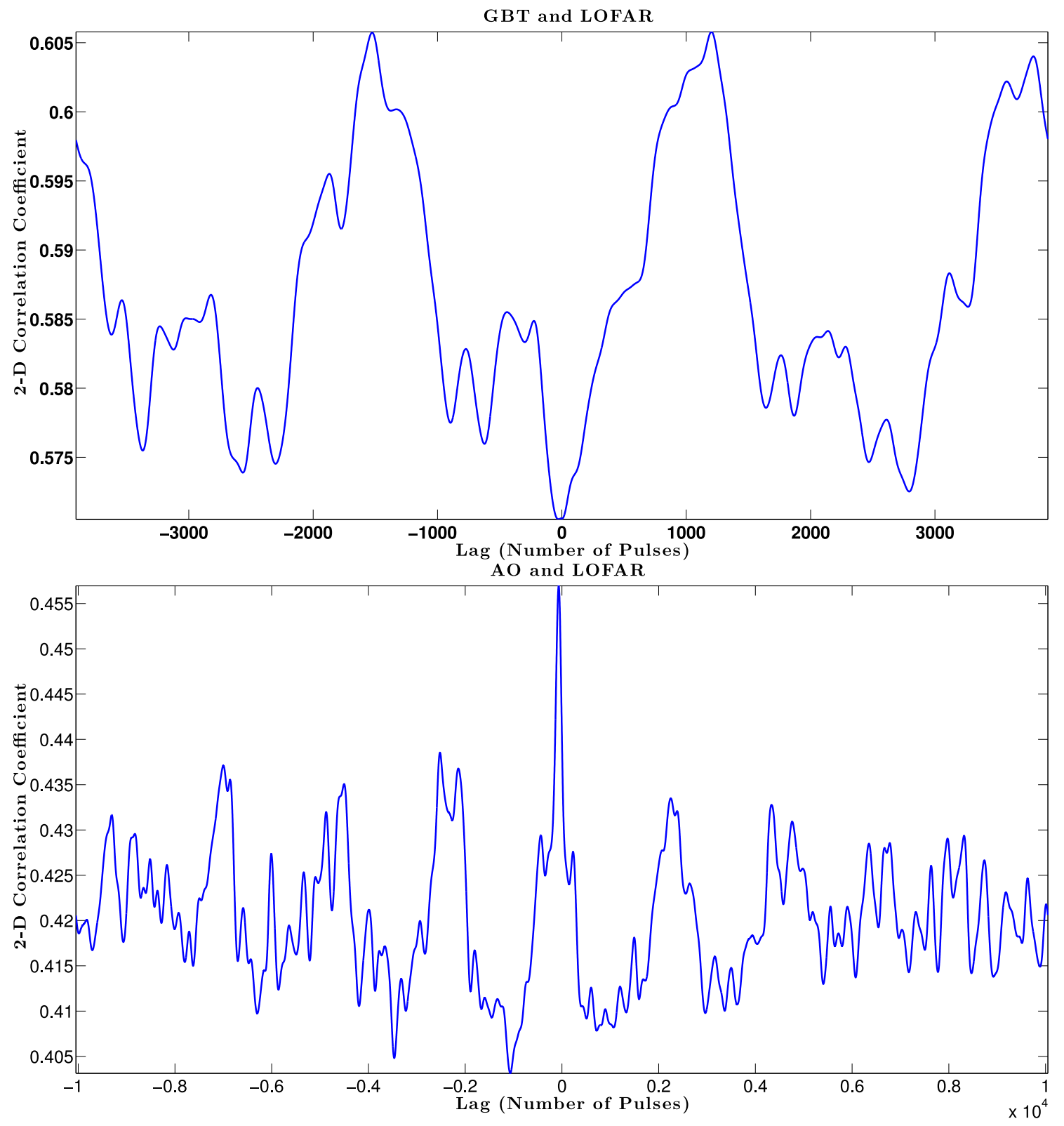

Figure 3.4: Cross-correlation function (CCF) plot of Top Panel: 820/150 MHz and Bottom Panel: 327/150 MHz. The plot clearly brings out the correlation of burst mode in $327 / 150 \mathrm{MHz}$ and the anti-correlation in 820/150 MHz. 

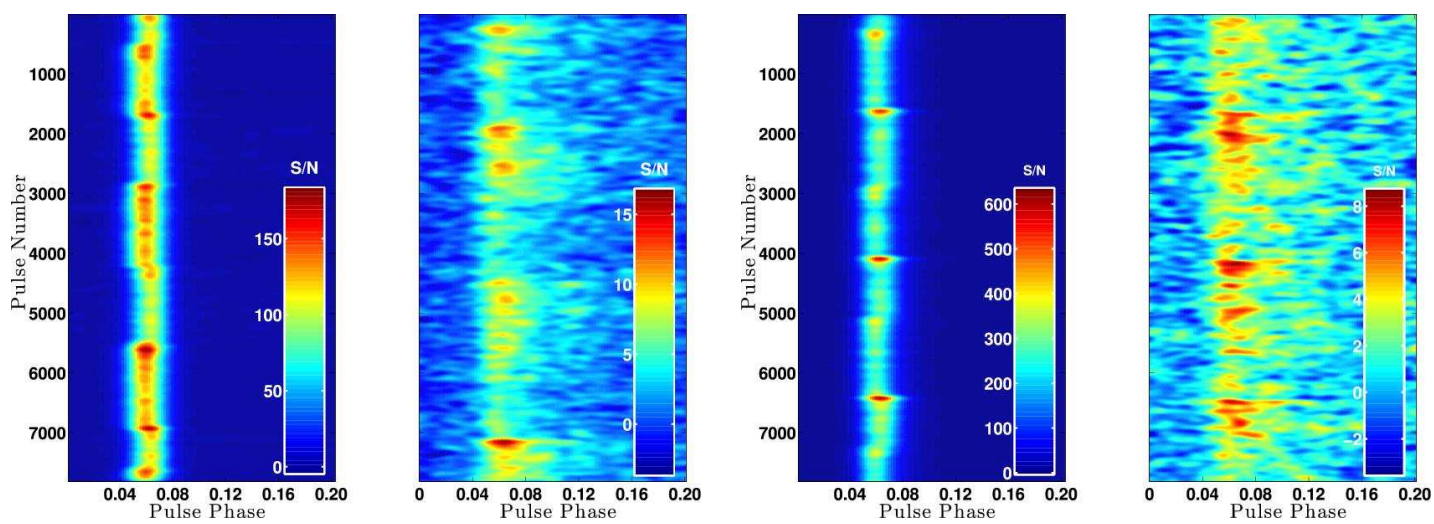

Figure 3.5: Simultaneous radio observations of PSR B0611+22 showing 820/150 MHz observations (left) and 327/150 MHz observations (right). The negative $\mathrm{S} / \mathrm{N}$ arises due to fluctuations of off-pulse noise below zero mean. Both datasets are of the same duration. Each dataset has been smoothed by using a 2-D Gaussian kernel of same dimensions (see text for details).

Fig. 3.6.

\subsubsection{Spectral turnover}

As we had multi-frequency data, we investigated the spectral behavior of this pulsar. Using the modified radiometer equation (Lorimer \& Kramer 2012), the flux density

$$
S=\frac{\beta T_{\mathrm{sys}} A}{G N_{\mathrm{bin}} \sigma_{\mathrm{off}} \sqrt{\Delta \nu N_{p} t_{\mathrm{obs}}}},
$$

where $A$ is the area under the pulse, $N_{\text {bin }}$ is the total number of phase bins in the profile, $N_{p}$ is the number of polarizations, $t_{\mathrm{obs}}$ is the total integration time of each phase bin of the pulse profile, $\beta$ is the correction factor for digitization, $\sigma_{\text {off }}$ is the rms of the noise in the pulsar timeseries and the rest of the parameters are as given in Table 3.1.

Estimating the flux of the LOFAR observations was not a straightforward pro- 

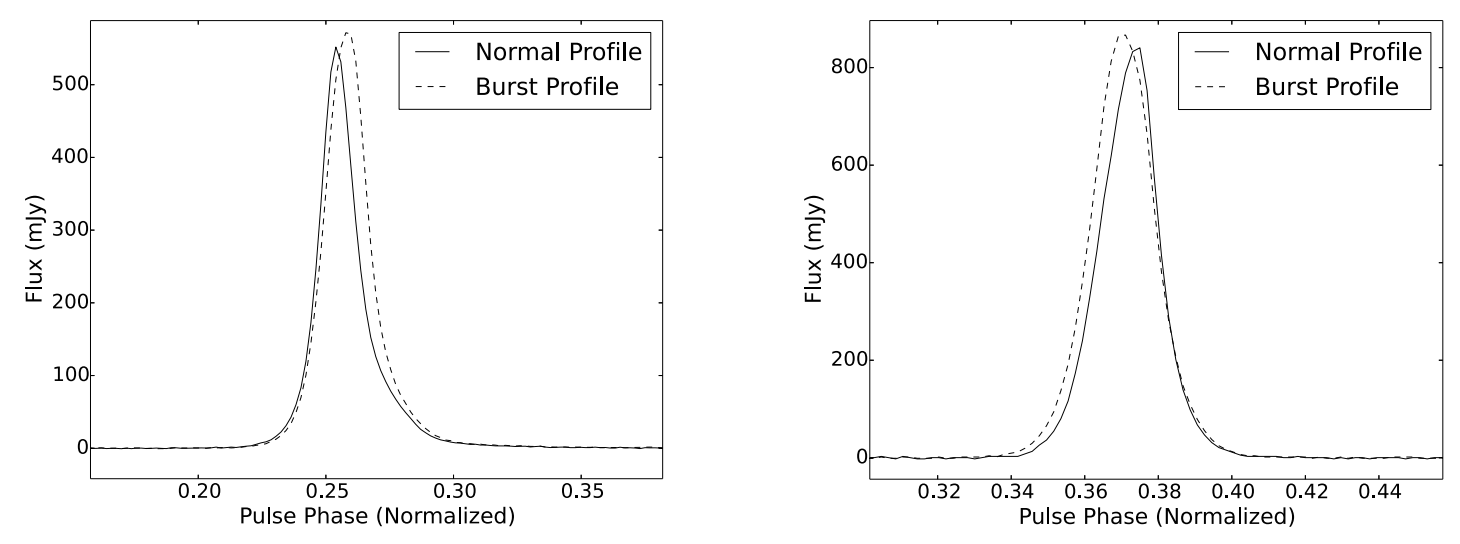

Figure 3.6: Profiles of normal and burst mode for $327 \mathrm{MHz}$ (left) and $820 \mathrm{MHz}$ (right).

cess. For FR606, which consists of antennas without moving parts, the temperature is strongly dependent on frequency, while the gain depends on frequency and on source position on the sky (elevation and azimuth). To calibrate the observed flux, we have used software described in detail by Kondratiev et al. (2015). The software produces flux density scaling factor using Eq 3.1 for each pulse and frequency channel. In order to properly estimate $G$ and $T_{\text {sys }}$, the software makes use of the Hamaker beam model (Hamaker, 2006) and mscorpol $^{3}$ by Tobia Carozzi to calculate Jones matrices of the HBA antenna response for a given frequency and sky coordinates. The HBA antenna temperature, $T_{A}$, is derived from CasA measurements done by Wijnholds \& van Cappellen (2011). The background sky temperature, $T_{s k y}$, is taken from a sky map at $408 \mathrm{MHz}$ by Haslam et al. (1982), scaled to the HBA frequency as $\nu^{-2.55}$ (Lawson et al., 1987). The error on the flux density calculation is $50 \%$ and can be attributed to e.g. error on system parameters, beam jitter due to the propagation in the ionosphere or strong sources contributing through the side

\footnotetext{
${ }^{3}$ https://github.com/2baOrNot2ba/mscorpol
} 
lobes of the beam. Detailed discussion on error calculation is provided by Bilous et al. (2015).

To obtain reliable flux estimates, it was important to consider all the biases that are introduced in this analysis due to the interstellar medium. The first effect we considered is interstellar scattering. The profiles in Fig. 3.1 suggest that at no frequency is the scattering tail a significant fraction of the pulse period. Therefore, scattering does not significantly alter any flux estimates; hence we decided to not compensate for scattering in the analysis of this chapter. Yet, a detailed treatment of the scattering will be discussed in another upcoming paper. Pulsar flux is also modulated by the free electron content along the line of sight. These modulations occur due to refractive interstellar scintillation (Gupta et al., 1994) which has timescale of the order of days and diffractive interstellar scintillation (Lewandowski et al., 2011) which can be of the order of minutes. Refractive scintillation will have little effect on our analysis as the timescales are much larger than the average observation length. However, since we were comparing our fluxes with those from Lorimer et al. (1995) and since those fluxes were measured twenty years ago, we decided to check whether refractive scintillations would affect our spectrum. We calculated the flux of the pulsar at $1400 \mathrm{MHz}$ from an observation done few years ago for (Seymour et al., 2014) and found that flux to match with the Lorimer et al. (1995) flux indicating that refractive scintillation may not dramatically modulate the flux. To check if the diffractive scintillations were biasing our flux estimates, we used the NE2001 electron distribution model (Cordes \& Lazio, 2002b) to calculate the diffractive scintillation timescales at 150, 327 and $820 \mathrm{MHz}$. The values we obtained were 46, 65 


\begin{tabular}{lc}
\hline $\begin{array}{l}\text { Frequency } \\
(\mathrm{MHz})\end{array}$ & $\begin{array}{c}\text { Flux } \\
(\mathrm{mJy})\end{array}$ \\
\hline 150 & $88 \pm 44$ \\
327 & $13.8 \pm 0.8$ \\
408 & $\mathbf{2 9} \pm 1$ \\
820 & $16.1 \pm 1.7$ \\
1408 & $\mathbf{2 . 2} \pm 1$ \\
\hline
\end{tabular}

Table 3.2: Flux densities calculated at different frequencies for PSR B0611+22. The values in bold are taken from Lorimer et al. (1995).

and $100 \mathrm{~s}$, respectively, which were small compared to the total integration time and the timescale of intrinsic variation of pulsar flux. Hence, we could use the pulse profiles as they were for further analysis.

The flux densities at various frequencies are listed in Table 3.2. The values suggest that the pulsar spectrum does not follow the standard power law model. To make sure that the $327 \mathrm{MHz}$ flux was not lower due to refractive scintillations, we calculated the flux of another observation at the same frequency which was used in Seymour et al. (2014). The calculated flux value was comparable to the new observations. In this analysis, we realized that the peak flux in our calculations did not match with peak fluxes in Seymour et al. (2014). After further cross-checks and verification, we conclude that the flux estimates in Seymour et al. (2014) are off by a factor of 16 as in their calculations, the time per bin ( $t_{\text {obs }}$ in Eq 3.1) is incorrect. Hence, we use the flux estimates computed here for further analysis. The turnover we see is in the middle of the frequencies where we see a temporal anti-correlation in the bursts which suggests that the physical processes responsible for the anti-correlation in bursts might also be responsible for this turnover. The other possibility is that the observed turnover is due to absorption of radio emission 


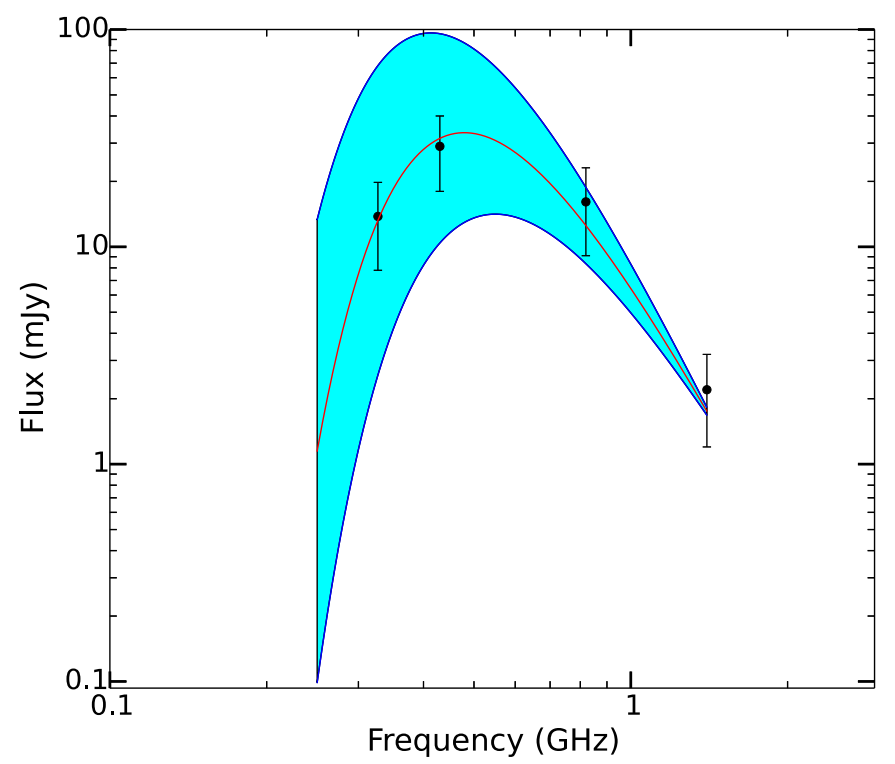

Figure 3.7: Modeled Spectrum for PSR B0611+22 using a free-free absorption model. The $150 \mathrm{MHz}$ flux has been excluded from the analysis because of high uncertainty in the flux measurement. The red curve is the best fit curve. The shaded region is enclosed within $\pm 1 \sigma$ curves. The $\pm 1 \sigma$ limits are derived from the fit. The electron temperature was set to $5000 \mathrm{~K}$ (Rajwade et al., 2016a). The derived emission measure is roughly $5 \times 10^{5} \mathrm{~cm}^{-6} \mathrm{pc}$. The reduced $\chi^{2}$ on the fit is 0.26 .

by an external absorber. The dense environment around the pulsar can lead to a turnover in the spectrum at a higher frequency than expected. This motivated us to model the spectrum using a free-free thermal absorption model to estimate the optical depth and the peak frequency (Lewandowski et al., 2015; Rajwade et al., 2016a). Past studies have detected H $\alpha$ emission (Bychkov \& Lebedev, 1979) in the region suggesting the presence of ionized gas. Fig. 3.7 shows the best fit model to the spectrum without the $150 \mathrm{MHz}$ observation. Although the dense ISM along the line of sight makes this model very tempting, the model fails to explain the whole spectrum of the pulsar. 


\subsubsection{Broadband flux density modulation}

To quantify the variation in the detected flux from the pulsar, we estimated the modulation index at different frequencies. Modulation index is a measure of pulse to pulse intensity fluctuation. To derive the intrinsic modulation indices $\left(m_{\mathrm{int}}\right)$ we used the method described in Kramer et al. (2003). First, we estimated the flux of each of the observations. Since we did not have calibrator observations, we used the radiometer equation (Lorimer \& Kramer, 2012) to obtain flux densities at different frequencies. Then, we normalized the time series by a $200 \mathrm{~s}$ running median to correct the pulsar signal for any possible effects of interstellar scintillation. Finally, the datasets were re-scaled to be consistent with the initial average flux density. For every observation, we integrated individual pulses to obtain the average pulse profile and calculated its flux density (see Table 3.2). After correcting the datasets for the effects of interstellar scintillation, for each one, we calculated the intrinsic modulation index

$$
m_{\mathrm{int}}^{2}=\frac{\left\langle(S-\langle S\rangle)^{2}\right\rangle}{\langle S\rangle^{2}},
$$

where, $\langle S\rangle$ is the mean flux density and $S$ is the measured flux density of a single pulse. We compared our results with results for other pulsars (Bartel et al., 1980; Kramer et al., 2003). It is clearly seen that $m_{\text {int }}$ decreases with frequency until a certain "cut off" frequency and rises again (see Fig. 3.8). Our results are consistent with other pulsars where a similar trend is observed (Maron et al., 2013). 


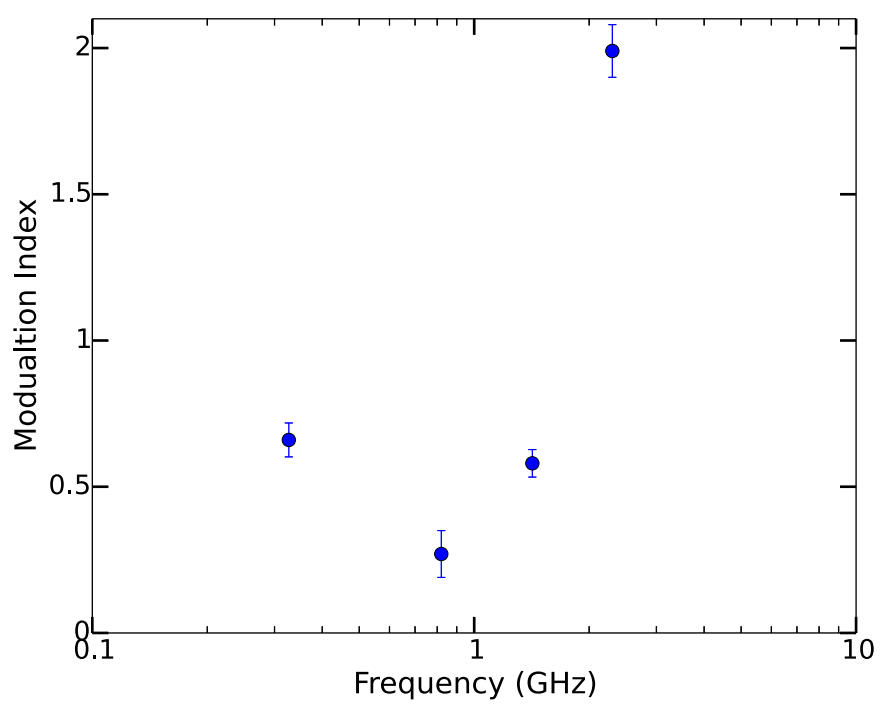

Figure 3.8: Modulation index $m_{\text {int }}$ as a function of frequency for PSR B0611+22. The modulation index at $2 \mathrm{GHz}$ was obtained from archival unpublished data from the Green Bank Telescope.

\subsubsection{X-ray flux upper limits}

We observed PSR B0611+22 for 33 ks using XMM Newton. We observed the pulsar using both PN CCD and MOS camera mounted on the telescope. We did not detect the pulsar but obtained an upper limit on the X-ray flux using the method described in Lorimer et al. (2012). We obtained an upper limit on the count rate of $\sim 5 \times 10^{-4}$ counts $\mathrm{s}^{-1}$ at $99 \%$ confidence level. Then, assuming a non-thermal emission from the pulsar with a photon index $\Gamma \approx 2$ (Pavlov et al., 2009) and using the publicly available software $\mathbf{W E B P I M M S}^{4}$, we obtained a limit on the unabsorbed X-ray flux, $F_{0.3-8 \mathrm{keV}}^{\text {unabs }} \sim 2.7 \times 10^{-15} \mathrm{ergs}^{-2} \mathrm{sm}^{-1}$ assuming a neutral hydrogen column density $N_{H}$ of $3.1 \times 10^{21} \mathrm{~cm}^{-2}$, estimated using $10 \%$ ionization fraction. The obtained upper limit is less than the flux reported for PSR

\footnotetext{
${ }^{4}$ https://heasarc.gsfc.nasa.gov/cgi-bin/Tools/w3pimms/w3pimms.pl
} 
B0943+10 (Hermsen et al., 2013) using the same non-thermal model for fitting. From this flux, we estimated an upper limit of X-ray luminosity, $L_{X}=4 \pi d^{2} F_{X}^{\text {unabs }}$ to be $<2.7 \times 10^{30} \mathrm{ergs} \mathrm{s}^{-1}$. From there, we were able to obtain an upper bound

on the X-ray efficiency $\eta_{0.3-8 \mathrm{keV}} 10^{-5}$ by assuming an $\dot{E}=10^{34} \mathrm{ergs} \mathrm{s}^{-1}$. This upper limit has an errorbar of roughly $50 \%$ given the uncertainties in the distance to the source.

\subsection{Discussion}

\subsubsection{Quasi-stable magnetosphere?}

A detailed understanding of the physics of pulsar magnetospheres has presented a major challenge to astronomers over the past four decades. Recently, new observations have shed some light on pulsar emission physics. Lyne et al. (2010) reported a correlation between the spin-down rate and pulse profile changes in a sample of mode changing pulsars. They concluded that the magnetosphere switched between multiple quasi-stable states of emission. This hypothesis was bolstered by recent radio and X-ray observations of PSR B0943+10, which showed that the thermal X-ray emission was anti-correlated with the radio emission (Hermsen et al., 2013).

Based on Rankin's model of radio emission from neutron stars (Rankin, 1983), PSR B0611+22 is a single core component pulsar. Later, Lyne \& Manchester (1988) and Johnston et al. (2008) classified this pulsar as a partial cone based on the polarization position angle sweep across the profile. Partial cone pulsars are known 
to exhibit broadband phenomena like nulling and mode changing (Bhat et al., 2007; Gajjar et al., 2014).

We observed that in PSR B0611+22, during the burst, the phase of the pulse shifts slightly, which agrees with the results in Seymour et al. (2014). In the $820 \mathrm{MHz}$ observation, the bursting occurred when the pulse phase shifted towards the leading edge. On the other hand, the bursting occurred when the pulse phase shifted towards the trailing edge in the $327 \mathrm{MHz}$ observations. This opposing behavior at different frequencies leads to an anti-correlation when compared to the simultaneous $150 \mathrm{MHz}$ data. Though PSR B0611+22 seems to be a mode changing pulsar, the frequency dependence of the mode change is not explained by current mode changing models and observations (Rankin, 1983). Kern (2000) claims that phase offset in the bursting profile occurs due to the periodic existence of a conal component along with the core component. Though the model is able to explain the slight increase in the width of the pulse profile in bursting mode, it fails to account for the different direction of phase offset at different frequencies. The observations presented here suggest that the spectral indices of the two components are different. Therefore, if the components preserve their phase, this manifests itself as an anti-correlation in the two simultaneous datasets We intend to do follow-up observations with a wider frequency coverage before making any claims on the model of emission of this pulsar.

At 327 and $820 \mathrm{MHz}$, we observed quasi-periodic bursting behavior. Though the period can be derived from the peak in the power spectrum, the lack of harmonics suggests that we need to sample more bursts to better characterize the periodicity. This also puts forth a question of whether similar pulsars like PSR 
J1752+2359 (Gajjar et al., 2014) and PSR J1939+2213 exhibit such behavior.

The non-detection of the pulsar in the X-ray waveband contradicts the predictions made in Seymour et al. (2014), based on assuming the 1\% X-ray efficiency of PSR B0943+10. However, it is consistent with average efficiency of $8 \times 10^{-5}$ found in Vink et al. (2011) for old (age $>17 \mathrm{kyr}$ ) pulsars and their nebulae. This shows that the pulsar is a weak X-ray emitter. The assumption of efficiency of 1\% in Seymour et al. (2014) is based on PSR B0943+10 which has different spin down properties compared to PSR B0611+22. Hence, it is not surprising that the efficiency is dramatically different.

\subsubsection{Flux Density Spectrum}

From our flux density estimates, we were able to conclude that the spectrum of PSR B0611+22 does not exhibit single power law behavior. This could be due to free-free thermal absorption along the line of sight. Recently, Kijak et al. (2011) and Dembska et al. (2014) have shown that these so called giga hertz peaked (GPS) spectra pulsars have peculiar environments in close vicinity of the pulsar or potential absorbers lying along the line of sight. This clearly suggests a turnover might occur at a frequency higher than $100 \mathrm{MHz}$. Recent modeling and simulations (Lewandowski et al., 2015; Rajwade et al., 2016a) suggest that free-free absorption in high density plasma surrounding supernova remnants, pulsar wind nebulae or dense, cold, partially ionized gas is responsible for high frequency turnovers. Using this idea, we modeled the spectrum with a free-free thermal absorption model. The 
main caveat in this result is that the model cannot explain the whole spectrum of the pulsar because of the higher flux value of $150 \mathrm{MHz}$ observation even though free-free thermal absorption seems like a promising explanation to the non-power law behavior given the dense environment along the line of sight toward the pulsar.

Since the model cannot explain the whole spectrum, external absorption scenario seems unlikely. The other possibility we considered is that the irregular spectral behavior could be caused due to the intrinsic variations in the pulsar itself. The different spectral indices of the two components within the partial cone could appear as a turnover in the flux density spectrum. Future observations at frequencies higher than $820 \mathrm{MHz}$ will shed some light on this phenomenon. Also, it will be important to verify the flux densities at LOFAR frequencies for future studies.

We also calculated the modulation indices at various frequencies. It can be seen (Fig. 3.8) that there is a turnover at $\sim 1 \mathrm{GHz}$. This result agrees with what is seen in other pulsars (Maron et al., 2000). The turnover in the modulation indices is mainly caused by decreasing average pulsar energy. Therefore the number of socalled pseudo nulls (no detection due to inadequate receiver sensitivity) increases with frequency.

\subsection{Conclusions}

We have carried out a detailed analysis of simultaneous radio and X-ray observations of pulsar PSR B0611+22. The multi-frequency data reveal a wealth of information about the emission characteristics of this pulsar. The bursting be- 
havior varied across the radio band with a quasi-periodic characteristic at all frequencies. The $327 / 150 \mathrm{MHz}$ and $820 / 150 \mathrm{MHz}$ simultaneous observations show an anti-correlation in bursting. We leave modeling this unusual behavior to a later paper. Future polarimetric studies of both modes will help in discerning the emission physics of this pulsar. Moreover, we obtained a flux density spectrum from the radio observations of this pulsar. The spectrum shows a turnover at higher frequencies. We considered free-free thermal absorption by the surrounding ISM as a possible explanation but such model cannot explain the flux density at $150 \mathrm{MHz}$. From the X-ray non-detection, we obtained an upper bound on the X-ray luminosity and X-ray efficiency of the pulsar. The X-ray non-detection shows that the X-ray efficiency is low and consistent with X-ray efficiencies measured for other similarly aged pulsars. 


\section{Chapter 4}

\section{Detecting fast radio bursts at decametric wavelengths}

The work presented in this chapter is published as: Kaustubh Rajwade and D. R. Lorimer, 'Detecting fast radio bursts at decametric wavelengths', 2017, MNRAS, Vol. 465, Iss. 2, p 2286

\subsection{Introduction}

Broadly speaking, the FRB source models fall into two categories: those of a catastrophic nature which would only be seen once (e.g., prompt emission from a gamma-ray burst; Yamasaki et al., 2016) or those with the possibility to repeat (e.g., giant pulses from Crab-like pulsars; Cordes \& Wasserman, 2016; Cordes et al., 2004). So far, the only source known to repeat is FRB 121102 (Spitler et al., 2016). In the light of these recent discoveries, and to try to shed light on the origins of FRBs a number of groups are carrying out extensive radio surveys at sub-GHz frequencies (Karastergiou et al., 2015; Caleb et al., 2016; Deneva et al., 2016). To date, however, the $0.7-0.9 \mathrm{GHz}$ detection of FRB 110523 remains the only source found below $1 \mathrm{GHz}$ (Macquart \& Kanekar, 2015).

Lyutikov et al. (2016) argues that a lack of detections could be due to absorption in an ionized medium along the line of sight. Recent discoveries suggest low scattering in all FRBs which precludes a local plasma in the vicinity of the pro- 
genitor to explain their high DMs (Macquart \& Kanekar, 2015; Macquart \& Koay, 2013). Kulkarni et al. (2015) argue for a young magnetar model with circum-dense medium around the star which can explain the high DM and the non-detections at lower frequency due to free-free absorption. The non-detections can also be ex-

plained by young neutron star progenitor within an expanding supernova remnant shell with hot ionized filaments (Piro, 2016).

In this chapter, we present a detailed analysis of the aforementioned absorption and scattering models. We use the approach to investigate the significance of non-detections in three recently completed surveys to constrain the spectral index of the burst for each model. Based on these constraints we make predictions for FRB detections from CHIME, UTMOST and HIRAX. Connor et al. (2016) make optimistic predictions for these upcoming low frequency surveys based on single FRB detection in the $0.7-0.9 \mathrm{GHz}$ band. Here, we present predictions on the FRB detection rates based on different models of flux mitigation in the ISM. The plan for this rest of this chapter is as follows. We describe our analysis methods in $\S 2$. In $\S 3$, we describe our results and discuss their implications in $\S 4$.

\subsection{Methods}

This section describes the methodology used for obtaining upper limits on FRB predictions with CHIME under different astrophysical scenarios. Our study is motivated by our recent work on modeling gigahertz peaked spectrum pulsars via free-free absorption (Rajwade et al., 2016b). Here, we investigate what could 
happen to an FRB that is absorbed or scattered and how that affects detectability with CHIME and UTMOST. We will begin by making use of the recent null results of FRB detections in the ongoing UTMOST survey (Caleb et al., 2016), the Arecibo drift scan survey (AO327; Deneva et al., 2016) and the $145 \mathrm{MHz}$ LOFAR survey (Karastergiou et al., 2015). We also considered the 155 and $182 \mathrm{MHz}$ surveys with the Murchison Widefield Array (MWA) (Tingay et al., 2015; Rowlinson et al., 2016) in our analysis. However, since the flux limits for those surveys are higher than the LOFAR survey, the spectral index constraints are less stringent than the LOFAR survey. We do not include results from single-pulse searches in the ongoing Green Bank North Celestial Cap (GBNCC) survey (Stovall et al., 2014) in this analysis. The constraints from these results are presented in Chawla et al. (2017).

\subsubsection{Flux-redshift relationship and baseline model}

Our methodology builds upon that used by Karastergiou et al. (2015) in their LOFAR survey, to include the effects of free-free absorption and scattering. Following these authors, we assume that FRBs are standard candles and the energy output from the source follows a power law with respect to frequency (see, e.g., Lorimer et al., 2013). Then, the peak flux density

$$
S_{\text {peak }}=\frac{L \int_{\nu_{1}(1+z)}^{\nu_{2}(1+z)} E_{\nu^{\prime}} d \nu^{\prime}}{4 \pi D(z)^{2}\left(\nu_{2}-\nu_{1}\right) \int_{\nu_{\text {low }}^{\prime}}^{\nu_{\text {high }}^{\prime}} E_{\nu^{\prime}} d \nu^{\prime}}
$$

where $L$ is the bolometric luminosity, the pulse energy $E_{\nu^{\prime}} \propto \nu^{\prime \alpha}$ for some spectral index $\alpha$ and source frame frequency $\nu^{\prime}=(1+z) \nu$ at redshift $z$ and luminosity 
distance $D(z)$. Also in the source frame, $\nu_{\text {low }}^{\prime}$ and $\nu_{\text {high }}^{\prime}$ are the frequency bounds in which the source emits. Following Lorimer et al. (2013), we assumed $\nu_{\text {high }}^{\prime}=10 \mathrm{GHz}$ and $\nu_{\text {low }}^{\prime}=10 \mathrm{MHz}$. The observed frequency band is defined by $\nu_{1}$ and $\nu_{2}$ and is different for each survey under consideration. We will discuss the implications of this standard-candle assumption in $§ 4.4 .3$.

Our implementation of the earlier study by Karastergiou et al. (2015) to place constraints on FRB spectral indices is summarized in Fig. 4.1 and described below. Since the distance scale for FRBs is not well known, we consider two scenarios: (i) a "cosmological model" for which the maximum redshift $z_{\max }=0.75$ (see, e.g., Lorimer et al., 2013); (ii) an "extragalactic model" for which the characteristic distance is 100 Mpc (i.e. $z_{\max }=0.025$; see, e.g. Lyutikov et al. 2016). Having chosen one of these two scales, we then derive the FRB rate versus redshift relationship by assuming an FRB population with constant density per unit comoving volume out to $z_{\max }$. At the chosen value of $z_{\max }$ this rate matches, by definition, the rates published by Crawford et al. (2016) based on FRB surveys at Parkes. Using this curve, for each of the other surveys under consideration (i.e. LOFAR, AO327 and UTMOST), we can compute the number of FRBs expected as a function of redshift by multiplying the rate-redshift relationship by the appropriate survey sky and time coverage. The resulting number versus redshift curves then lead to a limiting redshift $z_{\text {lim }}$ for each survey. This limiting redshift is defined to be that at which $<1 \mathrm{FRB}$ is predicted to be seen in each survey. An example of one such calculation is shown for UTMOST in the left panel of Fig. 4.2.

Next, for each of the source models A-F described in detail below, we choose 


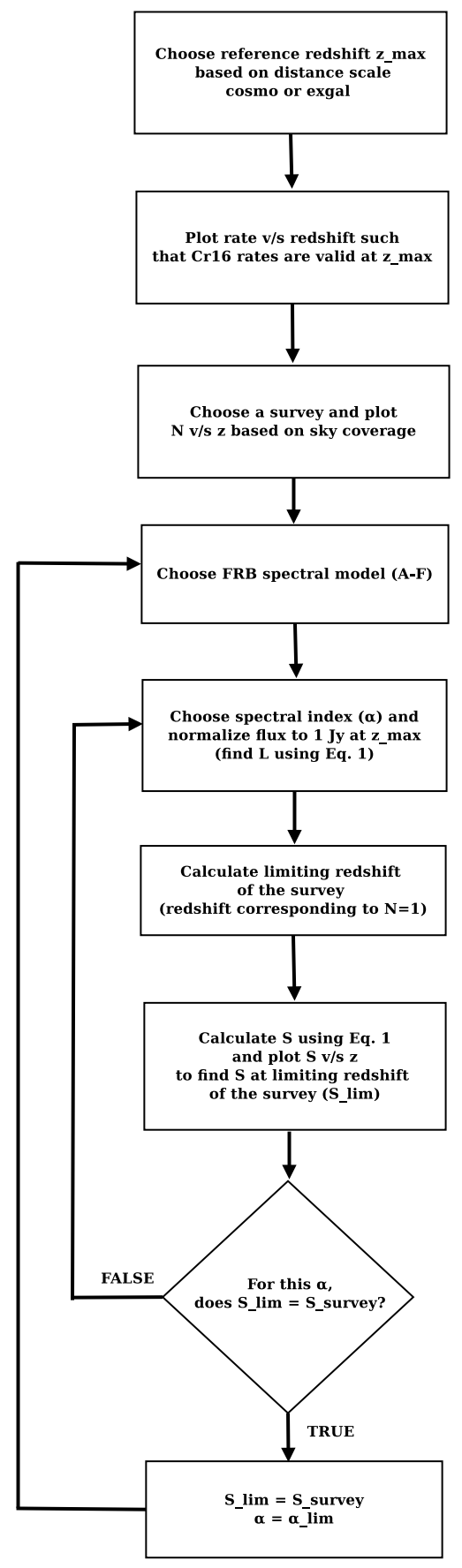

Figure 4.1: Flow diagram showing the logical flow of our analysis procedure for placing constraints on the spectral index. For further details, see $§ 4.2 .1$. 
a spectral index $\alpha$ and, using Eq. 1, find the corresponding value of $L$ such that $S_{\text {peak }}=1 \mathrm{Jy}$ at $z_{\max }$. The $1 \mathrm{Jy}$ reference flux is approximate, and motivated by the results of Thornton et al. (2013). Our results turn out to be insensitive to the exact value adopted here. For each of the surveys under consideration, we calculate the corresponding flux at the survey's redshift limit, i.e. $S_{\text {peak }}\left(z_{\lim }\right)$ and iterate until the spectral index is found where $S_{\text {peak }}\left(z_{\text {lim }}\right)$ equals the survey flux limit. This spectral index is, by definition, the limiting value appropriate to the assumptions of that particular model and distance scale, and we refer to this lower limit as $\alpha_{\text {lim }}$.

Our baseline model, which follows this process using a simple power-law spectral behaviour amounts to a repeat of the analysis of Karastergiou et al. (2015). We refer to this case as model "A" henceforth and, as necessary, distinguish between the cosmological and extragalactic cases in the text. The relevant parameters used for each of these models and constraints obtained from them are given in Table 4.2 and discussed further in the sections below.

\subsubsection{FRB survey sensitivity model}

From radiometer noise considerations, if $W$ is the width of the FRB then, for a search in which the pulse is optimally match filtered by a top-hat pulse of peak flux density $S$, the signal-to-noise ratio

$$
\mathrm{S} / \mathrm{N}=\frac{S G \sqrt{W n_{\mathrm{p}} \Delta \nu}}{T_{\mathrm{sys}}}
$$


where $T_{\text {sys }}$ is the system temperature, $\Delta \nu$ is the bandwidth, $n_{\mathrm{p}}$ is the number of polarizations summed and $G$ is the gain. In all current FRB surveys, where incoherent dedispersion techniques are used to process the data, and in the context of our models DM depends on redshift, then there is a dispersive broadening effect that results in a dependence between survey sensitivity and redshift. To model this effect, we compute the effective width of the pulse

$$
W_{\text {eff }}=\sqrt{W_{\text {int }}^{2}+W_{\mathrm{DM}}^{2}+W_{\tau}^{2}}
$$

where $W_{\text {int }}$ is the intrinsic pulse width of the $\mathrm{FRB}, W_{\mathrm{DM}}$ is the intra-channel dispersion smear and $W_{\tau}$ is the additional broadening due to the finite sampling interval of the survey. To calculate $W_{\mathrm{DM}}$, we adopted a DM-redshift scaling from (Inoue, 2004) where $\mathrm{DM}=1200 z \mathrm{~cm}^{-3}$ pc. Using the standard expression for dispersion broadening (see, e.g., Lorimer \& Kramer, 2004), we have

$$
W_{\mathrm{DM}}=99.6 \mathrm{~ms}\left(\frac{z}{n_{\text {chan }}}\right)\left(\frac{\Delta \nu}{\mathrm{MHz}}\right)\left(\frac{\nu}{\mathrm{GHz}}\right)^{-3}
$$

where $n_{\text {chan }}$ is the number of frequency channels used for dedispersion. Future FRB surveys may well introduce high-speed algorithms to implement coherent dedispersion (see, e.g. Zackay \& Ofek, 2014), in which case $W_{\text {DM }}$ will not be necessary. To model the degradation due to incoherent dedispersion of current and near-future surveys, consider an "optimal survey" signal-to-noise ratio, $\mathrm{S} / \mathrm{N}_{0}$ which is obtained from Eq. 4.2 for the case for a top-hat pulse with height $S_{0}$ and width $W_{\text {int }}$. For a 


\begin{tabular}{lclcl}
\hline Survey & $\begin{array}{c}\text { Centre frequency } \\
(\mathrm{MHz})\end{array}$ & $\begin{array}{l}\text { Bandwidth } \\
(\mathrm{MHz})\end{array}$ & $\begin{array}{c}\text { Flux limit } \\
\mathrm{mJy}\end{array}$ & Reference \\
\hline UTMOST & 843 & 31.5 & 11000 & Caleb et al. (2016) \\
AO327 & 327 & 57 & 83 & Deneva et al. (2016) \\
LOFAR & 145 & 6 & 62000 & Karastergiou et al. (2015) \\
CHIME & 600 & 400 & 125 & Newburgh et al. (2014) \\
HIRAX & 600 & 400 & 24 & Newburgh et al. (2016) \\
\hline
\end{tabular}

Table 4.1: Table showing various parameters of different surveys. The system parameters of CHIME and HIRAX are estimated values (see text for details).

broadened pulse of width $W_{\text {eff }}$, energy conservation means that its peak flux density is $S_{0} W_{\text {int }} / W_{\text {eff }}$. It is straightforward to show that the $\mathrm{S} / \mathrm{N}$ of the broadened pulse is lower than $\mathrm{S} / \mathrm{N}_{0}$ by a factor of $\sqrt{W_{\text {int }} / W_{\text {eff }}}$. For an actual survey with a constant $\mathrm{S} / \mathrm{N}$ threshold, this amounts to an increase in the limiting peak flux density for detection by the reciprocal of this factor, so that the resulting limiting sensitivity

$$
S_{\mathrm{lim}}=S_{0} \sqrt{\frac{W_{\mathrm{eff}}}{W_{\mathrm{int}}}}=\frac{\mathrm{S} / \mathrm{N}_{\mathrm{lim}} T_{\mathrm{sys}}}{G W_{\mathrm{int}}} \sqrt{\frac{W_{\mathrm{eff}}}{n_{p} \Delta \nu}} .
$$

This expression is used when calculating the sensitivity curves throughout this chapter (see, e.g., the right panel of Fig. 4.2). Here $\mathrm{S} / \mathrm{N}_{\lim }$ is the limiting signal-to-noise ratio required for a detection in a given survey. Table 4.1 summarizes the essential observing parameters for each of the surveys considered in this chapter.

\subsection{Models for flux mitigation}

Radio signals propagating through the ISM are modulated by free electrons in the intervening medium. These interactions leave observational signatures in the received radiation at the earth. Some of these signatures (e.g. scattering, free-free 
absorption and scintillation) have been observed in various radio sources. FRBs, being astrophysical in nature, are subject to the same phenomena. It is therefore important to model these effects in detail before we draw any inferences about their intrinsic spectral indices and make predictions for future surveys. Below, we describe our mathematical models to characterize effects of scattering and free-free absorption.

\subsubsection{Models including free-free absorption}

As discussed by other authors (Kulkarni et al., 2015; Lyutikov et al., 2016), but not taken into account by Karastergiou et al. (2015), thermal absorption can significantly reduce FRB fluxes at lower frequencies. For this analysis, following our earlier work (Rajwade et al., 2016b), we assume

$$
E_{\nu^{\prime}} \propto \nu^{\prime \alpha} \exp \left(-\tau \nu^{\prime-2.1}\right)
$$

where, as described further by Rajwade et al. (2016b), the optical depth of the absorber

$$
\tau=0.082 T_{e}^{-1.35} \mathrm{EM} .
$$

Here $T_{e}$ is the electron temperature and EM is the emission measure of the absorber. Then the peak flux is computed by combining Eq. 4.1 and Eq. 4.6. We consider two cases for absorption: (i) cold, molecular clouds with ionization fronts for which $T_{e}=200 \mathrm{~K}$ and $\mathrm{EM}=1000 \mathrm{~cm}^{-6}$ (Lewandowski et al., 2015) (hereafter, model 
B); (ii) hot, ionized magnetar ejecta/circum-burst medium for which $T_{e}=8000 \mathrm{~K}$ and $\mathrm{EM}=1.5 \times 10^{6} \mathrm{~cm}^{-6}$ (hereafter, model $\mathrm{C}$ ). The value of EM for model $\mathrm{C}$ has been chosen from a range of values reported in Rajwade et al. (2016b), Kulkarni et al. (2015) and Lewandowski et al. (2015). 


\begin{tabular}{|c|c|c|c|c|c|c|c|c|c|c|}
\hline \multirow[t]{2}{*}{ Model } & \multirow[t]{2}{*}{$\begin{array}{c}T_{e} \\
(\mathrm{~K})\end{array}$} & \multirow[t]{2}{*}{$\begin{array}{l}\mathrm{EM} \\
\mathrm{cm}^{-6} \mathrm{pc}\end{array}$} & \multicolumn{2}{|c|}{ UTMOST } & \multicolumn{2}{|c|}{$\alpha_{\lim }$} & \multicolumn{2}{|c|}{ AO327 } & \multicolumn{2}{|c|}{$\begin{array}{c}z_{\lim } \\
\text { CHIME }\end{array}$} \\
\hline & & & cosmo & exgal & cosmo & exgal & cosmo & exgal & cosmo & exgal \\
\hline A & - & - & -0.70 & -1.30 & 0.0 & -0.50 & 0.70 & 1.25 & 1.54 & 0.10 \\
\hline B & 200 & 1000 & -0.80 & -1.30 & -1.0 & -2.10 & 0.50 & 1.10 & 1.56 & 0.10 \\
\hline $\mathrm{C}$ & 8000 & $1.5 \times 10^{6}$ & -1.50 & -2.50 & - & - & -0.30 & -2.85 & 1.64 & 0.09 \\
\hline $\mathrm{D}$ & - & - & -2.10 & -3.30 & -3.0 & -4.0 & -3.30 & -2.20 & 0.84 & 0.06 \\
\hline $\mathrm{E}$ & 200 & 1000 & -2.20 & -3.30 & -4.10 & -5.70 & -3.50 & -2.50 & 0.85 & 0.06 \\
\hline $\mathrm{F}$ & 8000 & $1.5 \times 10^{6}$ & -2.70 & -4.50 & - & - & -4.50 & -6.45 & 0.82 & 0.05 \\
\hline
\end{tabular}

Table 4.2: Model parameters and resulting spectral constraints from the various surveys considered. From left to right, we list the model, assumed electron temperature $\left(T_{e}\right)$ and emission measure $(\mathrm{EM})$, limiting spectral index $\left(\alpha_{\text {lim }}\right)$ for the three published surveys (LOFAR, AO327 and UTMOST). For the future CHIME survey, we list the limiting redshift ( $\left.z_{\text {lim }}\right)$ predicted by our models. The "cosmo" and "exgal" columns give results from the two different distance scales assumed: "cosmological" $(z=0.75)$ and "extragalactic" $(z=0.025)$ as defined further in the text. 


\subsubsection{Models including multi-path scattering}

Multi-path scattering due to free electrons in the ionized medium along the line of sight to the observer can cause a reduction in the measured flux at the telescope. Scattering manifests itself as an exponential tail in the radio pulse of the FRB. FRBs that have been discovered so far, show only a modest amount of scattering: for the $17 \mathrm{FRBs}, 10$ of them have scattering measurements and 7 have them have upper limits (Cordes et al., 2016). Hence, we computed the scattering timescale by taking the average of the published values (estimates and upper limits) of these 17 sources. For sources with upper limits, conservatively, we assumed those values as measured values when taking the average. We obtained a mean scattering timescale of $\sim 8.1 \mathrm{~ms}$ at $1 \mathrm{GHz}$. We note that if we assume the scattering timescales for sources with upper limits as half of the upper limitvalues, we get a average timescale of $\sim 6.7 \mathrm{~ms}$ which is also a high value. Using the most conservative value, the scattering timescale $\tau_{s}$ can be computed for any frequency $\nu$ via the $\nu^{-4}$ scaling law (Bhat et al., 2004) as opposed to $\nu^{-4.4}$. The non-Kolmogorov scaling exponent is due to fact that the diffraction length scale is smaller than the inner scale of the wavenumber spectrum (see Bhat et al., 2004, , and references therein). Assuming that energy of the burst is conserved, if the pulse scatters with a timescale of $\tau_{s}$, the

width increases and hence, the measured flux reduces by a factor of $\sqrt{1+\left(\tau_{s} / W_{\text {eff }}\right)^{2}}$ where $W_{\text {eff }}$ is the effective pulse width defined in the preceding section. Including this effect into our analysis, we introduce three final models. Model D has scattering with no free-free absorption, while models $\mathrm{E}$ and $\mathrm{F}$ have scattering in addition to 

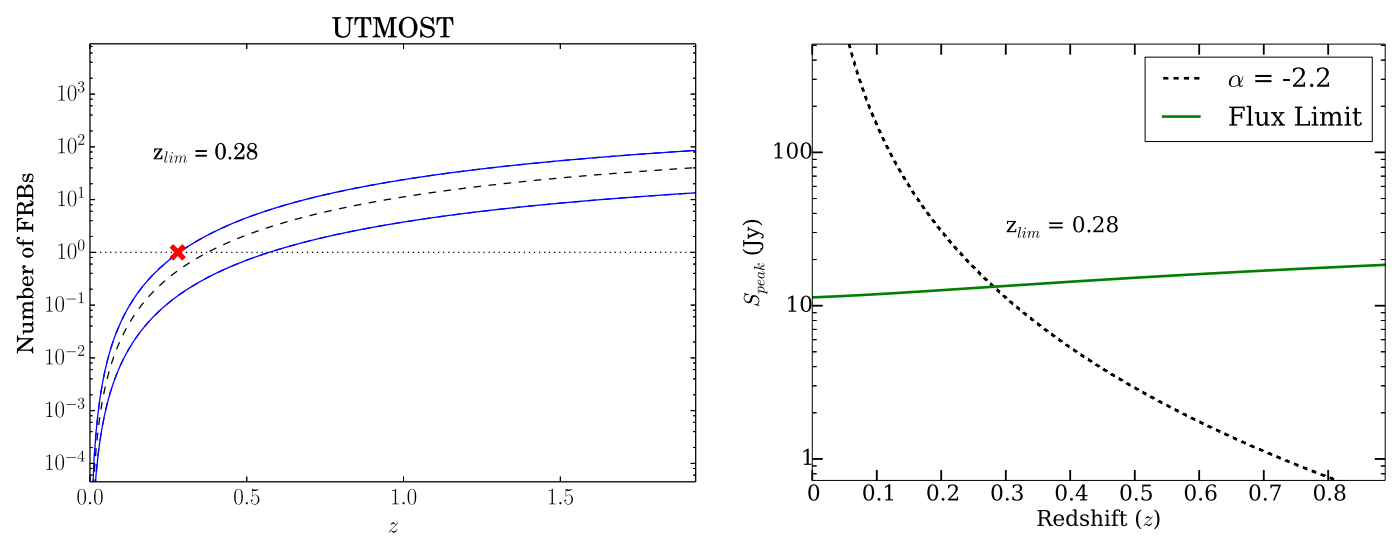

Figure 4.2: Left: Number of FRBs versus redshift for the UTMOST survey parameters Caleb et al. (2016). The curves indicate the Crawford et al. (2016) rates with $99 \%$ bounds. The horizontal line corresponds to one FRB. The intersection of the horizontal line and the upper bound of the curve is shown by the red cross at $z=0.28$. Right: peak flux versus redshift for UTMOST survey for model E (dashed curve). The solid line shows the flux limit of the UTMOST survey. The intersection of the two curves is denoted by $z_{l i m}$. Note the non-linear dependence of flux limit with redshift for both surveys shown here is due to the impact on intra-channel dispersion broadening upon sensitivity (see $\S 2.2$ for details).

the respective absorption parameters adopted for models $\mathrm{B}$ and $\mathrm{C}$.

\subsection{Results}

\subsubsection{Spectral index constraints}

Taking into account all the factors discussed in the previous section, the results of our analysis are collected for models $\mathrm{A}-\mathrm{F}$ in in Table 4.2. For each of these models, we constrained the spectral indices assuming each of the two distance constraints in turn. A graphical illustration of this process is shown for model E as an example in Fig. 4.2 where we show the constrained spectral index for one of the models for the UTMOST survey (Caleb et al., 2016). As mentioned previously, our baseline 
model (A) is an update on the results of Karastergiou et al. (2015) using the more recent rate FRB rate estimates from Crawford et al. (2016). In our analysis, which also includes the non-detections in UTMOST and AO327, the most constraining power-law spectral index for this model is $\alpha>0.7$ for the cosmological distance scale from AO327. The most constraining spectral index $(\alpha>1.25)$ is obtained from the AO327 survey if the extragalactic distance scale is applied to this survey.

In model $\mathrm{B}$, where we go beyond the simple power-law spectral dependence and include free-free absorption with cold molecular clouds, we find only a modest change in the results for model A for AO327 and UTMOST but as expected a greater deviation at the LOFAR frequency band where spectral turnover effects are more severe. The LOFAR survey does not in fact provide any constraints on the spectral index for models $\mathrm{C}$ and $\mathrm{F}$, where a hot ionized medium is assumed. These models predict flux densities below the survey threshold for essentially all values of $\alpha>-10$. The corresponding $\alpha_{\text {lim }}$ values are therefore not listed in Table 2 .

The spectral index constraints become much weaker when the effects of interstellar scattering are incorporated in models D, E and F. For model D, with scattering but no free-free absorption is assumed, the UTMOST null results only bound $\alpha>-2.2$ for the cosmological case and the AO327 results bound $\alpha>-2.2$ for the extragalactic case. When free-free absorption and scattering are considered in models $\mathrm{E}$ and $\mathrm{F}$, these constraints are diminished further. 


\subsubsection{FRB rate predictions for future surveys}

Fig 4.3 shows the predicted detection rates for UTMOST, CHIME and HIRAX for the two distance scales considered. The vertical line corresponds to the redshift limit of the survey for all models A-F. These predictions were obtained from the spectral constraints on each model obtained in the previous section, and computing the sensitivity of each survey as described below.

In modeling the sensitivity of CHIME, we assume that the gain $G=2 \mathrm{~K} \mathrm{Jy}^{-1}$ and system temperature $T_{\text {sys }}=50 \mathrm{~K}$ remain constant over the band. We also assumed a single CHIME beam of width 1.5 by 90 degrees (Bandura, private communication). Using Eq 4.2, we obtained the optimum flux limit of $0.125 \mathrm{Jy}$ for a $5 \mathrm{~ms}$ duration burst. For the scattering scenario, we used the frequency weighted average value of $\tau_{s}$ over the whole CHIME band. We obtained $\tau_{s}=92.2 \mathrm{~ms}$. For each of the models described in Table. 4.2, and the using the constraint on the spectral index from the UTMOST survey, we plotted the peak flux versus redshift using

Eq. 4.2. For each model at the constrained spectral index, we obtained the $z_{\text {lim }}$ which is the redshift where the peak flux of the FRB is equal to the flux sensitivity limit of CHIME as shown in Fig. 4.4. Then, using the expected sky coverage of CHIME and scaling the Crawford et al. (2016) rate with the comoving volume, we obtained the predicted number of FRB detections per day versus redshift as shown in left panel of Fig. 4.3. The ordinate of the point at which the $z_{\text {lim }}$ for each model intersects the curve and the bounds gives the predicted number of FRB detections per day for that given model. We investigated the yield for HIRAX surveys with 

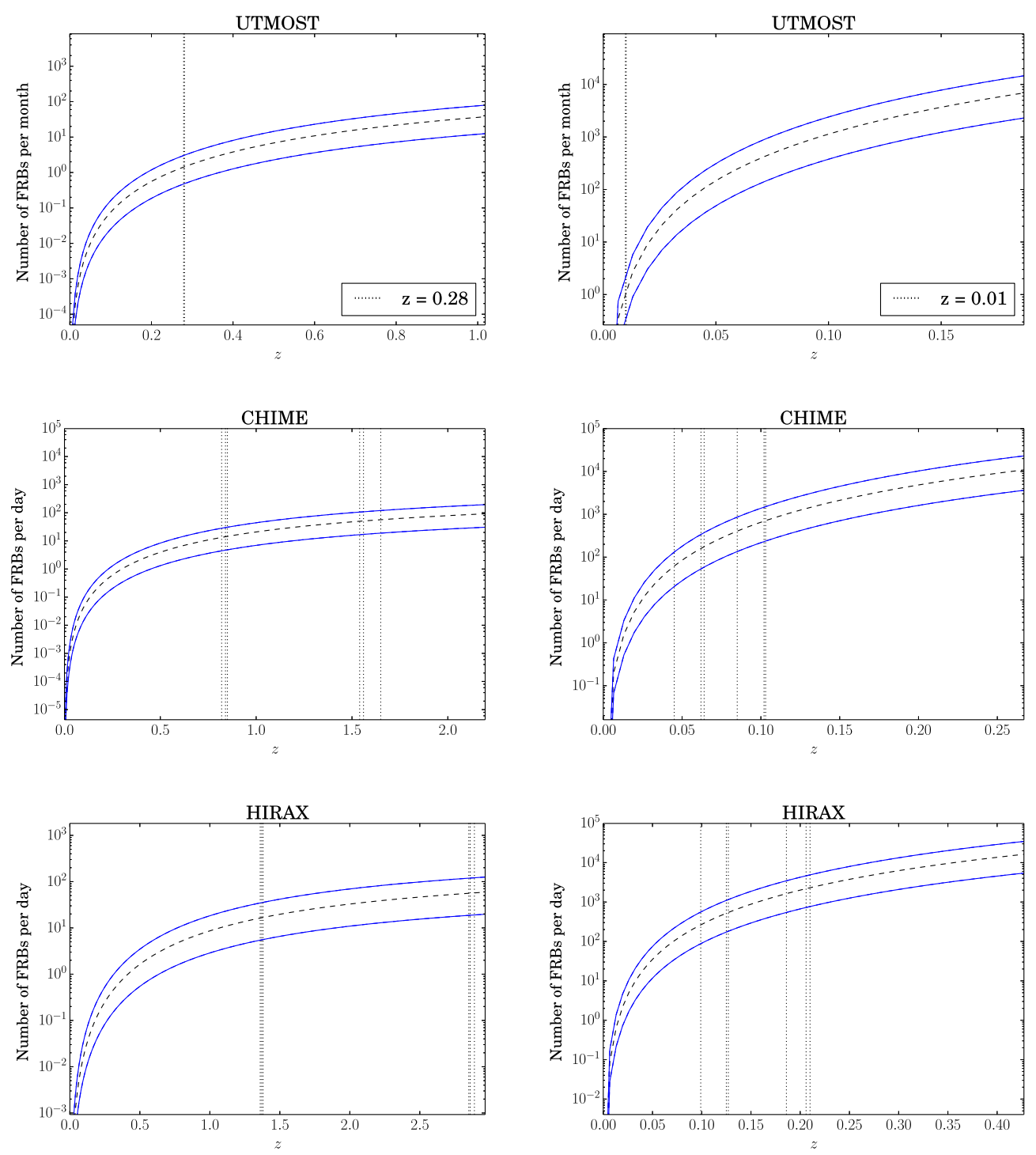

Figure 4.3: The number of FRBs predicted per day/month as a function of redshift for various surveys. The black dashed curve is the number of FRBs per day based on the (Crawford et al., 2016) rates. The blue curves are the $99 \%$ upper and lower bounds on the black dashed curve. Left panels show predictions for the cosmological case while the right panels show predictions for extragalactic case. In predictions for CHIME (cosmological case), from left to right, the vertical lines correspond to models F,D,E,A,B and C respectively while they correspond to models F,E,D,C,B and A respectively for the extragalactic case. Similarly for HIRAX, the vertical lines from left to right correspond to models $\mathrm{D}, \mathrm{E}, \mathrm{F}, \mathrm{A}, \mathrm{B}$ and $\mathrm{C}$ respectively for the cosmological case and F,E,D,C,B and A respectively for the extragalactic case. In case of UTMOST, the single vertical line corresponds to all models for their respective constrained spectral index at the limiting redshift of the survey. The ordinate of intersection of the vertical line and the curves gives the predicted number for each model. 


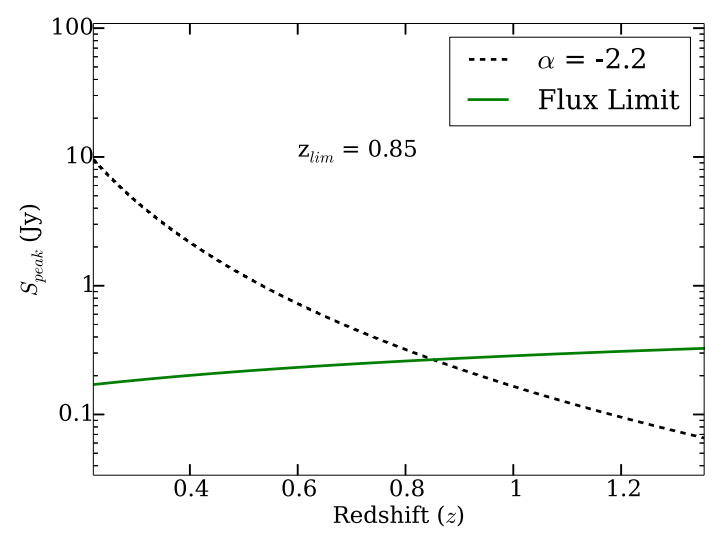

Figure 4.4: Peak flux versus redshift predicted for model E assuming the nominal parameters of CHIME. The intersection of the survey limit and the curve gives the limiting redshift probed by CHIME for this model.

identical parameters as the ones used for CHIME except for $G=10.5 \mathrm{~K} \mathrm{Jy}^{-1}$. The analysis suggests that CHIME will be able to detect from 30-100 FRBs per day depending on the model for the cosmological case while the yield increases by an order of magnitude ( 150-1000 FRBs per day) for the extragalactic case due to the sharp dependence of rates with redshift. Similarly, HIRAX will be able to detect 50-100 FRBs per day for the cosmological case and 700-4000 FRBs per day for the extragalactic case.

\subsubsection{Caveats}

Our analysis has a number of simplifying assumptions about the nature of FRBs. In this section, we investigate the sensitivity of our results to these assumptions. A key simplification we have made is to assume that FRBs are standard candles. Recent models and surveys for FRBs suggest that there might be distribution of luminosities for these bursts (see, e.g., Caleb et al., 2016; Vedantham et al., 
2016). Hence, we investigated the effect of FRBs having a range of luminosities. By definition, for a population of standard candles, all sources are detected out to a survey's redshift limit. This means that, for a distribution of luminosities, only those FRBs that are fainter than the currently assumed value will have any impact on the results. To investigate this, we repeated our analysis by reducing the luminosities by a factor of 10 from the value assumed above. This factor is motivated by the approximate distribution of energies in the study of Caleb et al. (2016). This exercise resulted in weaker constraints on the spectral index values for each model such that the $\alpha_{\text {lim }}$ values reported in Table 2 are reduced by factor of anywhere between 1.5 and 2 . Therefore, for a population with a range of luminosities in general, we would expect the constraints given in Table 2 to be reduced slightly. We also note that lowering the luminosities assumed necessarily results in lower predicted yields for future FRB surveys. For example, we found that our predictions for CHIME were reduced by up to a factor of 2 . In summary, a range of luminosities for the FRB population will tend to reduce the constraints on spectral index and lead to different survey yields. This complication only further highlights the value that future surveys will have in probing the FRB population.

The recent discovery of a repeating FRB (Spitler et al., 2016) provides some evidence that a neutron star scenario is the most plausible model for these bursts. If FRBs do originate from neutron stars, we detect the brightest pulses from them in the local universe. This constrains the distance to these sources to $z=0.025$ (i.e. 100 Mpc). We also investigated the effect of such an assumption and results are shown in Table 4.2 and Fig. 4.3. One would assume that given a smaller distance to the 
sources, CHIME would see more of them. The results agree with this conjecture. Fig. 4.3 suggests that even with models including scattering and free-free absorption, CHIME would see $~ 100$ FRBs per day if they were in the local universe.

In all of our calculations, we have implicitly assumed that the FRB rate is constant per unit comoving moving probed by the surveys. If the FRB rate traces the cosmological star formation rate (SFR), then we would expect the maximum number of sources to be found at $z=2(5.3 \mathrm{Gpc}$ ) (Madau \& Dickinson, 2014). Caleb et al. (2016) compared a sample population of FRBs based on the SFR to the observed sample and found a good match with different parameters of the observed sample although the pulse widths could not be accounted for. Given the current size of the FRB population, and difficulties in ascribing a distance scale, we regard this as a subtlety that is currently not well probed by the observations. We do, however, comment on a related factor that may impact future observations in the discussion below.

\subsection{Discussion}

Our results suggest that telescopes in the $0.4-1.0 \mathrm{GHz}$ band will make vital contributions to our understanding of FRBs. Even with free-free absorption and scattering playing a vital role in flux mitigation of FRBs, CHIME will be able to detect these bursts on a daily basis by the virtue of its extensive bandwidth and vast instantaneous sky coverage. We also looked into the possible caveats in the analysis and the effects those would have on the predictions for CHIME. Our investigation 

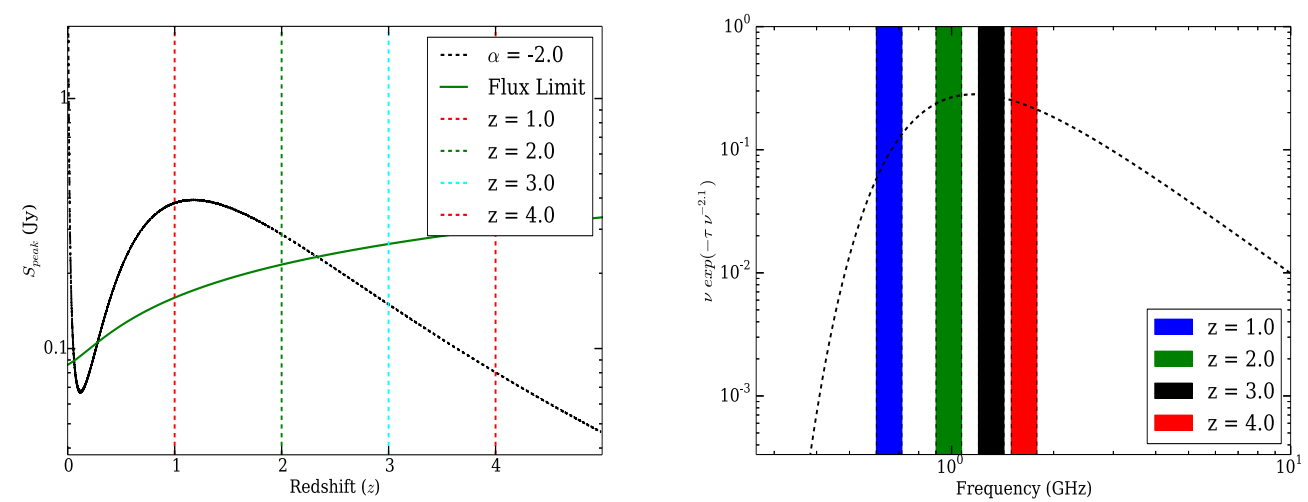

Figure 4.5: Left: peak flux versus redshift for the AO327 survey for model F illustrating the effect of absorption and Doppler shift of the observed frequency as described in the text. The black dashed line is the flux of the FRB. The different vertical lines correspond to different redshifts. In this case, we assumed $\alpha=-2, \mathrm{EM}$ $=3 \times 10^{6} \mathrm{~cm}^{-6} \mathrm{pc}$ and $T_{e}=8000 \mathrm{~K}$. Right: the different regions of the absorption spectrum probed by the survey at different redshifts. The different shaded regions correspond to the rest frame frequency probed by the survey at different redshifts.

suggests that with all the caveats considered, the lowest yield for a future CHIME survey is $\sim 30$ FRBs per day which is very optimistic compared to expected yield from other surveys. For example, the corresponding yield for future UTMOST observations is about 1-2 FRBs per month for future observations which makes it difficult to differentiate between the two models at the moment.

We also discussed certain caveats in our analysis (\$4.4.3) and how these assumptions affect the results. We found that a distribution in luminosities for FRBs, rather than a standard candle model assumed here, results in weaker constraints for the spectral indices of the population. Future surveys, however, will be excellent at probing the FRB luminosities through the dependence of luminosity on survey yield.

If the FRBs currently observed lie predominantly in the local Universe (i.e. have characteristic distances of $100 \mathrm{Mpc}$ ), then the large DMs cannot be accounted for by 
the Milky Way, host and IGM contributions. This discrepancy suggests that a large contribution to the DM comes from the local plasma around the source which favors models $\mathrm{C}$ and $\mathrm{F}$ as the most plausible scenarios describing these events. Assuming the parameters in model $\mathrm{C}$, we can estimate the linear size of the absorber around the source in order to produce the high DMs observed for FRBs. If we take the FRB with the highest known DM (FRB 121002) and place it at $z=0.025$ then, assuming model $\mathrm{C}$, we obtain a linear size of $\sim 1.4 \mathrm{pc}$. This is very similar to the parsec size high density filaments found in supernova remnants and magnetar ejecta (Lewandowski et al., 2015; Koo et al., 2007; Kulkarni et al., 2015). Thus, if future observations establish this distance scale for the FRB population, it should be possible to better constrain the model of absorption and the progenitor.

During the course of this work, we observed an interesting trend in the FRB flux as a function of redshift for observations in the $<1 \mathrm{GHz}$ band where models $\mathrm{C}$ and $\mathrm{F}$ predict an increase in flux density as a function of redshift (see, e.g., the left panel of Fig. 4.5). This behaviour is due to the Doppler shifting of a spectrum with a turn-over in its rest frame, which is a natural feature of the free-free absorption models. For sources at higher redshifts, we sample a different region in the spectrum of the source (see the right panel of Fig. 4.5). If the spectrum has a turnover, the peak flux increases as we sample the rising edge of the spectrum. At higher redshifts, the frequency band passes over the turnover resulting in a decrease in the peak flux as expected. As discussed in $\S 4.4 .3$, we have not included the potential increase in the FRB rate with redshift that is predicted in cosmological models invoking star formation (Madau \& Dickinson, 2014). If these models prove to be relevant in 
future, the aforementioned effect will be even stronger than seen in Fig. 4.5.

The constraints given in Table 4.2 can tell us about the nature of the FRB progenitors. The observed and predicted spectral indices suggest that FRB spectral indices are different from pulsar spectral indices which have a mean of -1.4 (Bates et al., 2014). Observations have suggested that at least some FRB spectral indices are positive (Spitler et al., 2016). Assuming a synchrotron source, the spectral index and the flux together can give us order of magnitude estimates about the magnetic field and effective electron temperature of the source (see for e.g Condon \& Ransom, 2016). For example, if FRBs truly have a positive spectral index at frequencies of $1 \mathrm{GHz}$, the results favor a compact source with large magnetic field that is perpendicular to the line of sight (e.g., as seen in magnetar bursts) since the frequency at which the source becomes optically thick is proportional to the magnitude of the magnetic field while a negative spectral index would suggest other synchrotron sources (e.g., giant pulses from neutron stars). A large sample size of these sources expected from CHIME and HIRAX will definitely help to alleviate the problem.

In summary, we have carried out a detailed analysis of possible FRB source populations and the expected yield from ongoing and future radio surveys below $1 \mathrm{GHz}$, based on results from the previous surveys. The previous results help in constraining the spectral index of the burst although no inference on the emission model can be drawn currently. Even with the most stringent model, in which spectral turnovers are expected in the observing band, CHIME is expected to see FRBs very frequently. Similar results are expected to be seen by HIRAX. The yields of 
CHIME, HIRAX and UTMOST will undoubtedly lead to a large sample that will provide great insights into the nature of and emission mechanism of these enigmatic sources. 


\section{Chapter 5}

\section{Detecting pulsars in the Galactic center}

The work presented in this chapter is submitted as: Kaustubh Rajwade, D. R. Lorimer, Loren Anderson, Detecting pulsars in the Galactic Centre, MNRAS

\subsection{Introduction}

Understanding the stellar populations in the Galactic Center (GC) region, and how they relate to the central supermassive black hole ( $\left.\operatorname{Sgr} A^{*}\right)$, is a major goal of modern astrophysics. The central few parsecs of the Galaxy are known to consist of large molecular complexes and have high stellar densities compared to the rest of the Galactic disk (see, e.g., Schödel et al., 2007).

Motivated by the promise of finding pulsars orbiting Sgr $A^{*}$, there have been multiple surveys of the GC region (Johnston et al., 2006; Macquart et al., 2010; Deneva, 2010; Bates et al., 2011). These surveys are typically conducted at frequencies higher than $\sim 1 \mathrm{GHz}$ to reduce the impact of interstellar scattering, which is known to cause potentially significant pulse broadening along lines of sight to pulsars in the inner Galaxy (Cordes \& Lazio, 1997). To date, no pulsars have been found in the $\mathrm{GC}$ region, which we define in this chapter to be within 1 pc of $\mathrm{Sgr} \mathrm{A}^{*}$ (i.e. an

angular offset of $25^{\prime \prime}$ for $D_{\mathrm{GC}}=8.3 \mathrm{kpc}$ ). The discovery of a magnetar (Eatough et al., 2013; Mori et al., 2013) has brought the problem of pulsars in the GC to 

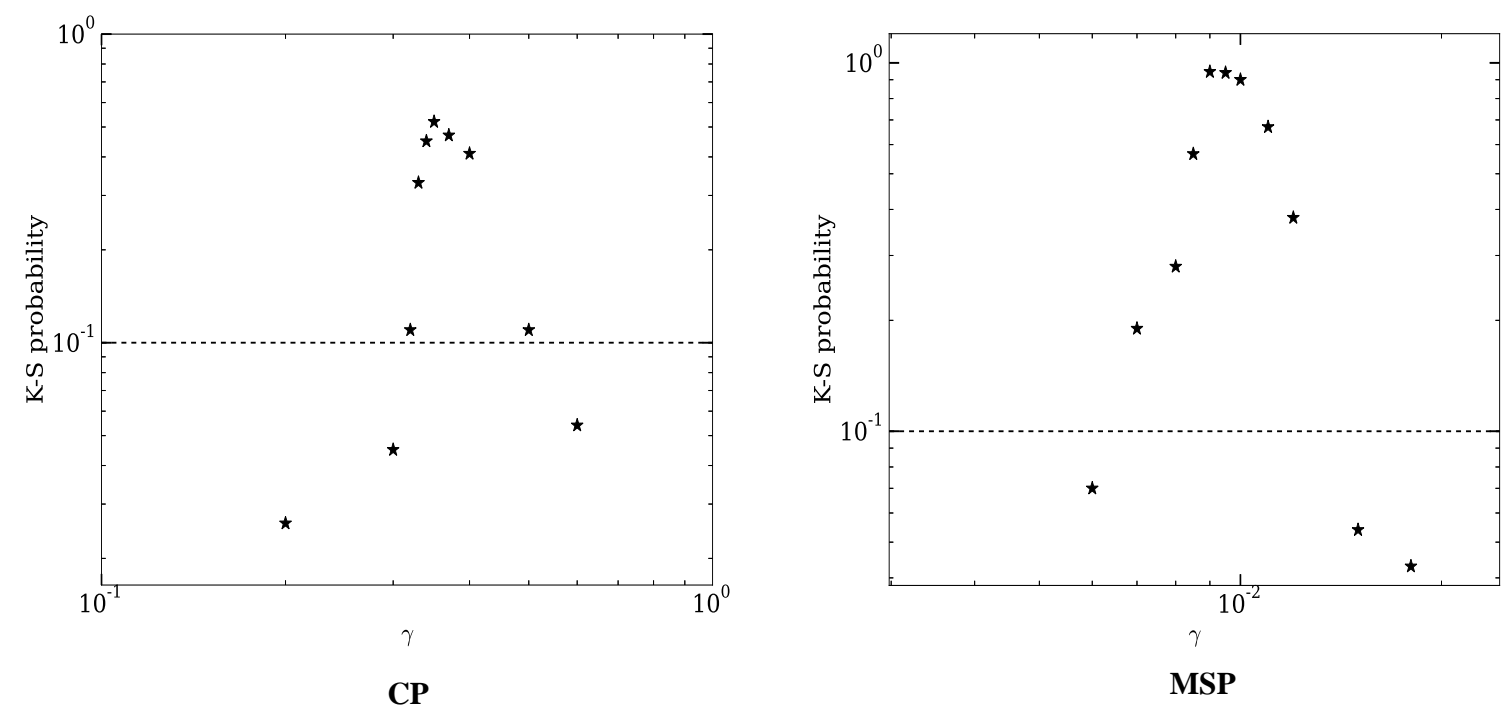

Figure 5.1: K-S probabilities of the pseudo-luminosity distributions of observed and simulated samples versus $\gamma$. The horizontal line in each plot, shown for reference, represents a K-S probability of 0.1 .

the fore again. Recently, Macquart \& Kanekar (2015) proposed that the neutron star population of the GC is dominated by millisecond pulsars (MSPs). They also claimed that more sensitive, high frequency surveys in the future would be able to detect MSPs in the GC. Though a MSP population has been predicted in the past, the results of Macquart \& Kanekar (2015) are based on the pseudo-luminosity distribution of the known pulsar population sample, which has an inherent pseudoluminosity bias since we only detect the brightest pulsars. In this chapter, we try to answer questions regarding the GC pulsar population by modeling the GC environment and accounting for observational selection biases. We simulate a pulsar population in the GC environment and study the effect of the GC environment on pulsar flux densities. We find the optimum frequency for future surveys based on the results of the simulation. 


\subsection{Simulations}

To place constraints on the number of pulsars in the GC, we simulated synthetic populations of pulsars using the PsrPopPy package (Bates et al., 2014), a python module based on the psrpop code developed earlier for population synthesis of pulsars (Lorimer et al., 2006). The inferred parameters from the known pulsar population in the Galaxy are biased due to various selection effects (see, e.g., Faucher-Giguère \& Kaspi, 2006). These effects are accounted for by PsrPopPy (see Bates et al., 2014, for details). PsrPopPy generates synthetic pulsar populations based on a set of pulsar parameters. These are then searched for in a simulated pulsar survey based on past survey parameters to determine the subset of pulsars that are theoretically detectable.

To briefly explain how we perform our simulations, we draw different physical properties of a pulsar like period, magnetic field, age and birth velocity from different assumed distributions. Then, for a given pulsar, the physical parameters described above are evolved to the given age resulting in the final evolved values for the simulated pulsars. Then, we calculate the luminosities of these evolved pulsars and using their final positions in the Galaxy, we calculate the flux. Then, a check is performed to see if a given pulsar is brighter than the sensitivity of the survey in concern. If it is, then the pulsar is added to the list of detected pulsars and this process goes on until the total number of pulsars is equal to the number of pulsars actually detected in that survey. Details of the exact calculations and different dis-

tributions used are given in Bates et al. (2014). We had to make some modifications 
to the code to accommodate the simulation of MSPs since the original code was not designed for MSP population synthesis. The distributions used and the assumptions made for MSP simulations are given in Table. 5.1.

We considered populations of canonical pulsars (CPs) and millisecond pulsars (MSPs) in our analysis with PsrPopPy (Bates et al. 2014). For both cases, we simulated the populations using the pseudo-luminosity scaling with period and period derivative. Following previous authors, we parameterize the pseudo-luminosity $L$ in terms of period $P$ and period derivative $\dot{P}$ as a power law:

$$
L=\gamma P^{\alpha} \dot{P}^{\beta},
$$

where $\alpha, \beta$ and $\gamma$ are model parameters. For simplicity, following Bates et al. (2014), we take $\alpha=-1.4$ and $\beta=0.5$ which physically links $L$ to be proportional to the square root of the pulsar's spin-down pseudo-luminosity. We used this relationship rather than using pulsars with known distances to compute the luminosity distribution because of the inherent bias towards brighter pulsars. The uncertainties on $\alpha$ and $\beta$ are reported in Bates et al. (2014). To ensure that errors on $\alpha$ and $\beta$ do not affect our results, we reran our simulations by changing one parameter by $1 \sigma$ and kept the other same. We observed that changing the parameters within the errors had little to no effect on the results as discussed later. To ensure that the properties of the simulated sample are comparable to the observed sample, we modified the constant of proportionality in this expression, $\gamma$, so that the pseudoluminosity of the simulated sample that is detected in a simulated Parkes survey 
matches the observed detected sample in the same survey, assuming that the properties of the pulsars in the GC are similar to the properties of detected pulsars. To achieve this, we simulated a population of CPs and MSPs for different $\gamma \mathrm{s}$ and ran a Kolmogorov-Smirnov (K-S) test on the pseudo-luminosity distributions of the simulated and the observed sample for both sub-populations. Since a K-S probability beyond $\sim 0.1$ implies that the model and observed distributions are statistically indistinguishable (see, e.g., Press et al., 2002), we obtain a range of $\gamma$ values for which the luminosities are consistent, as shown in Fig. 5.1. The best $\gamma$ was chosen for the case where we obtained the maximum K-S probability for the two detected populations. The best simulated populations were used for further analysis. The parameters used for simulation of both populations are given in Table 5.1. We note in passing here that the optimal values of $\gamma$ found here imply population-averaged luminosity values of $2.1 \mathrm{mJy} \mathrm{kpc}^{2}$ and $0.1 \mathrm{mJy} \mathrm{kpc}^{2}$ for CPs and MSPs respectively. Although our analysis does not make any distinction between solitary and binary MSPs, which appear to have different luminosities (Bailes et al., 1997; Burgay et al., 2013), it does clearly show that MSPs are intrinsically fainter radio sources than CPs. 
Table 5.1: Table showing the different model parameters used in PsrPopPy for simulation of the two pulsar populations. The values used in the simulation are adopted from previous Parkes surveys (Manchester et al., 2001; Edwards et al., 2001). Values in the parenthesis indicate 1- $\sigma$ uncertainties on the least significant digit. The term std stands for standard deviation. The parameters for different distributions for CPs have been adopted from Bates et al. (2014).

\begin{tabular}{|c|c|c|}
\hline Parameter & $\mathrm{CP}$ & MSP \\
\hline Radial distribution Model & Lorimer et al. (2006) & Lorimer et al. (2006) \\
\hline Initial Galactic z-scale height & $50 \mathrm{pc}$ & $50 \mathrm{pc}$ \\
\hline 1-D velocity dispersion & $265 \mathrm{~km} \mathrm{~s}^{-1}$ & $80 \mathrm{~km} \mathrm{~s}^{-1}$ \\
\hline Maximum initial age & 1 Gyr & 5 Gyr \\
\hline Luminosity parameter $\alpha$ & $-1.4(1)$ & $-1.4(1)$ \\
\hline Luminosity parameter $\beta$ & $0.50(4)$ & $0.50(4)$ \\
\hline Luminosity parameter $\gamma$ & 0.35 & 0.009 \\
\hline Spectral index Distribution & Gaussian & Gaussian \\
\hline$\langle\alpha\rangle$ & -1.4 & -1.4 \\
\hline$\sigma_{\alpha}$ & 0.9 & 0.9 \\
\hline Initial Spin period distribution & Gaussian & Log-Normal (Lorimer et al., 2015) \\
\hline$\langle P\rangle(\mathrm{ms})$ & 300 & - \\
\hline$\sigma_{P}(\mathrm{~ms})$ & 150 & - \\
\hline$\left\langle\log _{10} \mathrm{P}(\mathrm{ms})\right\rangle$ & - & 15 \\
\hline $\operatorname{std}\left(\log _{10} \mathrm{P}(\mathrm{ms})\right)$ & - & 56 \\
\hline
\end{tabular}


Pulsar spin-down model

Beam alignment model

Braking Index

Initial B-field distribution

$\left\langle\log _{10} \mathrm{~B}(\mathrm{G})\right\rangle$

$\operatorname{std}\left(\log _{10} \mathrm{~B}(\mathrm{G})\right)$

Observed sample size

Faucher-Giguère \& Kaspi (2006) Faucher-Giguère \& Kaspi (2006)

orthogonal

3

Log-normal 12

0.55

1065 orthogonal

3

Log-normal

8

0.55

39 


\begin{tabular}{lccclcl}
\hline Survey & Frequency & $\mathrm{T}_{\text {sys }}$ & $\mathrm{t}_{\text {int }}$ & $\mathrm{G}$ & $\mathrm{S} / \mathrm{N}_{\min }$ & $\Delta \nu$ \\
\hline & $(\mathrm{GHz})$ & $(\mathrm{K})$ & $(\mathrm{s})$ & $\left(\mathrm{K} \mathrm{Jy}^{-1}\right)$ & & $(\mathrm{MHz})$ \\
\hline Bates et al. 2011 & 6.5 & 40 & 1055 & 0.6 & 10 & 576 \\
Macquart et al. 2010 & 15 & 35 & 21600 & 1.5 & 10 & 800 \\
Johnston et al. 2006 & 8.4 & 40 & 4200 & 0.6 & 10 & 864 \\
SKA-MID & 5 & 30 & 50400 & 17.7 & 10 & 770 \\
ngVLA & 10 & 34 & 25200 & 22.4 & 10 & 8000 \\
\hline
\end{tabular}

Table 5.2: Basic parameters for previous and future pulsar surveys towards the GC. For each survey, we have used 2048 frequency channels.

We scaled the derived luminosities of the simulated population at $1.4 \mathrm{GHz}$ to different frequencies given in Table 5.4 for both populations assuming a normal distribution of spectral indices (Bates et al., 2013). Then, the corresponding observed flux density

$$
S=\frac{L_{\nu}}{D_{\mathrm{GC}}^{2}},
$$

where $L_{\nu}$ is the pseudo-luminosity at a frequency $\nu$ (see Chennamangalam \& Lorimer, 2014, for details) and $D_{\mathrm{GC}}$ is the distance to the GC which is assumed to be $8.3 \mathrm{kpc}$ (Bower et al., 2014).

We obtained flux densities for different frequencies from luminosities obtained in the simulations using Eq. 5.2. Then, using the models discussed in the subsequent sections, we multiplied the flux densities by the appropriate factors to account for the reduction due to three scenarios: (i) Scattering, where the flux density is reduced due to multi-path scattering between the source and the observer; (ii) Free-free absorption, where the radio flux density from the pulsar is absorbed by the intervening medium; (iii) both scattering and free-free absorption. Under these circumstances, we calculated the total number of CPs and MSPs detectable from previous and 

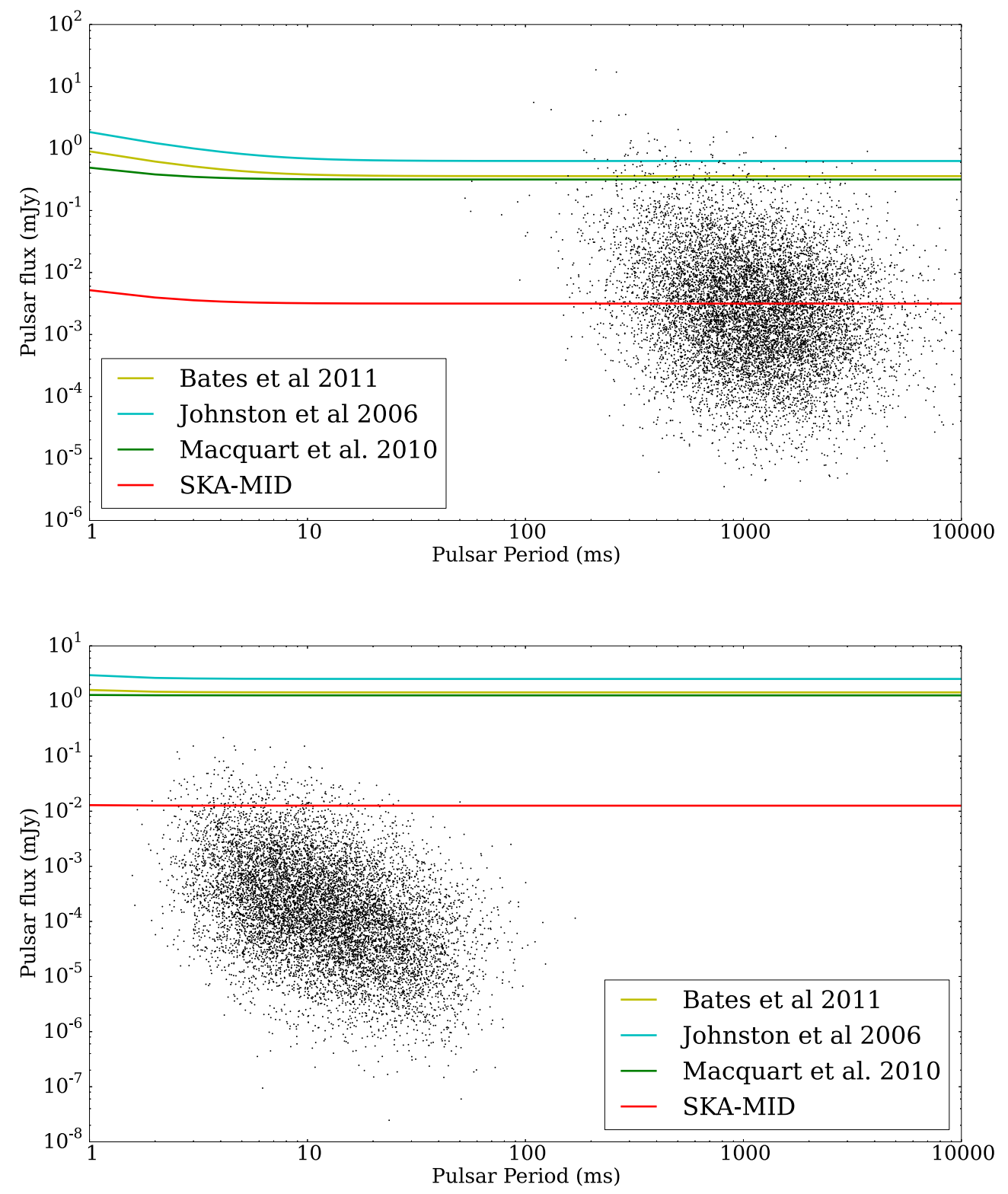

Figure 5.2: $1.4 \mathrm{GHz}$ mean flux density versus period for a synthetic population of 10000 pulsars at the GC for the baseline model (BL) showing the lack of sensitivity of previous surveys towards pulsars in the GC with no scattering and absorption. The top panel shows CPs while the bottom panel shows MSPs. Different lines indicate the survey sensitivities of past surveys and the SKA-MID survey. The parameters for SKA-MID survey are the expected parameters of the survey. The sensitivities of each survey have been scaled to $1.4 \mathrm{GHz}$ assuming a spectral index of -1.4 (Bates et al., 2013). The flux density limit curves for each survey correspond to a DM of $1780 \mathrm{~cm}^{-3}$ pc (corresponding to the DM of the GC Magnetar). The flux sensitivity limit for ngVLA is not shown since it almost overlaps with the SKA-MID flux limit. 


\begin{tabular}{lrrrrrrrrrr} 
Model & \multicolumn{1}{c}{\begin{tabular}{c}
\multicolumn{1}{c}{ Survey } \\
\end{tabular}} & \multicolumn{2}{c}{ A } & \multicolumn{2}{c}{ B } & \multicolumn{2}{c}{ C } & \multicolumn{2}{c}{ D } & \multicolumn{2}{c}{ E } \\
\hline & CP & MSP & CP & MSP & CP & MSP & CP & MSP & CP & MSP \\
\hline BL & 41 & 5000 & 51 & 10000 & 18 & 189 & 2 & 34 & 2 & 20 \\
WS & 41 & 10000 & 51 & 10000 & 18 & 555 & 2 & 44 & 2 & 20 \\
SS & 44 & - & 52 & - & 18 & 1111 & 3 & - & 2 & 417 \\
FF & 41 & 10000 & 51 & 10000 & 18 & 189 & 2 & 35 & 2 & 20 \\
FF+WS & 41 & 10000 & 51 & 10000 & 18 & 555 & 3 & 45 & 2 & 20 \\
FF+SS & 44 & - & 52 & - & 18 & 1111 & 3 & - & 2 & 417 \\
\hline
\end{tabular}

Table 5.3: Table showing the upper limits on the population for a null result in previous and future surveys. These are conservative limits since we use the lowest acceptable $\gamma$ values. The surveys considered here are: (A) Bates et al. (2011); (B) Johnston et al. (2006); (C) Macquart et al. (2010); (D) SKA-MID survey; (E) ngVLA survey. The models listed are: (1) the baseline (BL) model with no scattering or free-free absorption; (2) weak scattering (WS); (3) strong scattering (SS); (4) free-free absorption (FF); (5) free-free and weak scattering (FF+WS); (6) free-free and strong scattering $(\mathrm{FF}+\mathrm{SS})$. For example, in the baseline model for survey A, 244 CPs were detected implying an upper limit of 41 while 2 MSPs were detected implying an upper limit of 5000 .

future surveys using parameters for each survey. Then the upper limit on the GC population for each survey was calculated as the ratio of total pulsars simulated to the number of pulsars detected in the survey. For this analysis, where we report conservative limits on the GC pulsar populations, we used the lowest $\gamma$ value above a K-S probability of 0.1 . Those values are 0.32 for CPs and 0.007 for MSPs. Since the change in $\alpha$ and $\beta$ within $1 \sigma$ errorbars affected the number of detected pulsars in a given survey by a factor of $\sim 1$, we conclude that the change in those parameters does not affect our upper limits. The results of this analysis are shown in Table 5.3. Figure 5.2 shows the baseline simulation of CPs and MSPs with past survey sensitivities overlaid along with future SKA-MID and ngVLA (Carilli et al., 2015) surveys with assumed parameters of the telescope ${ }^{1}$. The results of the past

\footnotetext{
${ }^{1}$ https://www . skatelescope.org/wp-content/uploads/2012/07/SKA-TEL-SKO-DD-001-1_
} 
surveys along with the ngVLA and SKA-MID survey are shown in Table 5.3. From this it is evident that, even without considering any effects of the GC environment on the pulsar flux densities, the past surveys have been insensitive to the total pulsar population in the GC.

\subsubsection{Model}

In an attempt to make sense of the lack of pulsars in the GC found so far, we developed a model described below that takes account of multi-path scattering and free-free absorption effects on the pulsar signal. If $S_{0}$ is the intrinsic flux density of a pulsar at a frequency $\nu$, then the measured flux density at the telescope

$$
S_{\nu}=S_{0, \nu} \mathcal{S}(\nu) \mathcal{F}(\nu)
$$

where $\mathcal{S}(\nu)$ and $\mathcal{F}(\nu)$ are the flux density mitigation factors due to scattering and free-free absorption respectively. These factors are discussed in turn in the sections below.

\subsubsection{Free-free absorption}

Free-free absorption is known to bias flux density spectra of some pulsars (Lewandowski et al., 2015; Rajwade et al., 2016a). This is manifested by a turnover in pulsar spectra at frequencies of $\sim 1 \mathrm{GHz}$ (Kijak et al., 2007, 2011) which is different from the turnover seen at lower frequencies due to synchrotron self absorption (Sieber, 1973).

BaselineDesign1.pdf 
This phenomenon is normally observed in pulsars that lie in dense environments like pulsar wind nebulae or supernova remnants. Since the GC consists of dense, ionized gas and cold molecular gas with thin ionization fronts, we assume free-free absorption plays a part in reducing the flux density of an expected pulsar population at the GC. If $\tau$ is the optical depth along a given line of sight then, as we showed in Rajwade et al. (2016a), the observed flux density

$$
S_{\text {obs }, \nu}=S_{\text {ref }, \nu_{\text {ref }}}\left(\frac{\nu}{\nu_{\text {ref }}}\right)^{\alpha} \mathcal{F}(\nu)
$$

where

$$
\mathcal{F}(\nu)=\exp \left[-\tau_{\nu}\left(\frac{\nu}{\nu_{\mathrm{ref}}}\right)^{-2.1}\right]
$$

and $S_{\text {ref, } \nu_{\text {ref }}}$ is the pulsar's observed flux density at a reference frequency $\nu_{\text {ref }}$ at which $\tau_{\nu} \ll 1$. For a correction factor of order unity ${ }^{2}$, the optical depth

$$
\tau_{\nu}=0.082\left(\frac{\nu}{\mathrm{GHz}}\right)^{-2.1}\left(\frac{\mathrm{EM}}{\mathrm{cm}^{-6} \mathrm{pc}}\right)\left(\frac{T_{e}}{\mathrm{~K}}\right)^{-1.35} .
$$

For this analysis, following Pedlar et al. (1989), we adopt an emission measure EM $=5 \times 10^{5} \mathrm{~cm}^{-3} \mathrm{pc}$ and electron temperature $T_{e}=5000 \mathrm{~K}$ for the GC. Rajwade et al. (2016a) shows that this effect is smaller at frequencies greater than $\sim 1 \mathrm{GHz}$, which will be discussed later.

\footnotetext{
${ }^{2}$ This assumption is reasonable so long as $T_{e}>20 \mathrm{~K}$, which is the case in this work.
} 


\subsubsection{Scattering}

Given a flux density spectrum that is modified by free-free absorption in the GC region, we also need to consider the impact of multi-path scattering. Observations of scatter-broadened pulse profiles, which are typically in the form of a one-sided exponential, have long been known to be powerful probes of the physical composition and structure of the ISM (for a review, see e.g., Krishnakumar et al., 2015). Since the GC is a region with high stellar density and large amounts of molecular and ionized gas, a significant amount of scattering is expected for pulsars in this region. From Cordes \& Lazio (1997), for observations at some frequency $\nu$ and scattering due to a thin screen, the corresponding scattering timescale

$$
\begin{aligned}
t_{\mathrm{sca}}\left(\Delta_{\mathrm{GC}}\right) & =6.3 \mathrm{~s}\left(\frac{D_{\mathrm{GC}}}{8.5 \mathrm{kpc}}\right)\left(\frac{\theta_{\mathrm{GC}, 1 \mathrm{GHz}}}{1.3^{\prime \prime}}\right)^{2} \\
& \left(\frac{\nu}{\mathrm{GHz}}\right)^{-4}\left(\frac{D_{\mathrm{GC}}}{\Delta_{\mathrm{GC}}}\right)\left(1-\frac{\Delta_{\mathrm{GC}}}{D_{\mathrm{GC}}}\right) .
\end{aligned}
$$

In this expression, $D_{\mathrm{GC}}$ is the distance to the $\mathrm{GC}, \Delta_{\mathrm{GC}}$ is the distance of the scattering screen from the $\mathrm{GC}$ and $\theta_{\mathrm{GC}}$ is the angular broadening of $\mathrm{Sgr} \mathrm{A}^{*}$ scaled to a frequency of $1 \mathrm{GHz}$. We compute $\mathcal{S}(\nu)$ following the treatment in Cordes \& Lazio (1997) and Cordes \& Chernoff (1997). We assume pulses to be characterized by a Gaussian and convolve this with a one-sided exponential scattering function to broaden the pulse. In Fourier space, the amplitude of the harmonics will be the product of the Fourier transform of the Gaussian pulse and the scattering function. Since the scattering reduces the peak amplitude of the pulse, that manifests itself 


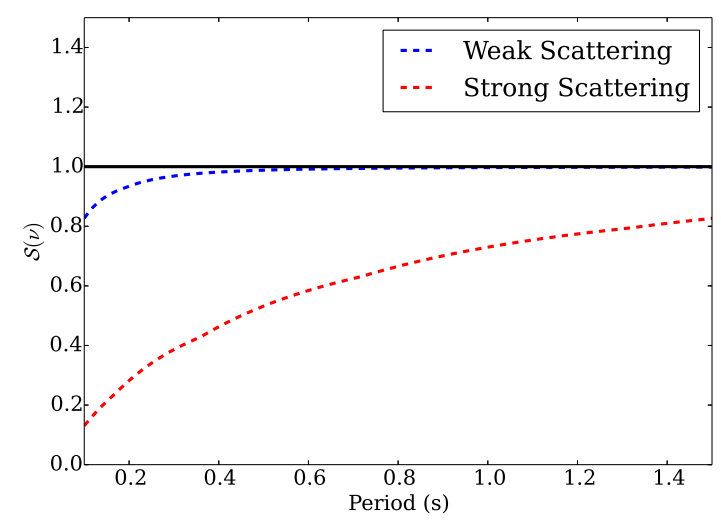

Figure 5.3: Scattering efficiency $(\mathcal{S}(\nu))$ as a function of period for CPs. The horizontal black line corresponds to $\mathcal{S}(\nu)=1$.

as a reduction in the efficiency of the survey. We define this efficiency

$$
\mathcal{S}(\nu)=\frac{\eta_{\mathrm{p}, \mathrm{sc}}}{\eta_{\mathrm{p}, \mathrm{std}}}
$$

where $\eta_{p, s c}$ is the pulsed fraction i.e. the sum of amplitudes of a set of harmonics divided by the amplitude of the DC component, for the scattered pulse and $\eta_{p, s t d}$ is the pulsed fraction of the standard Gaussian pulse (See Appendix B for details). The position of the scattering screen towards the GC is still uncertain. For this analysis, we assume the strong scattering scenario with the screen at $\sim 130$ pc (Cordes \& Lazio, 1997) and weak scattering with screen at $~ 6 \mathrm{kpc}$ from the GC (Bower et al., 2014). We did these calculations for CPs and MSPs for weak and strong scattering. Figure 5.3 shows the efficiency as a function of period for CPs. For this analysis, we used a constant duty cycle of 0.4 for MSPs (Kramer et al., 1998) and 0.05 for CPs (Rankin, 1993). 

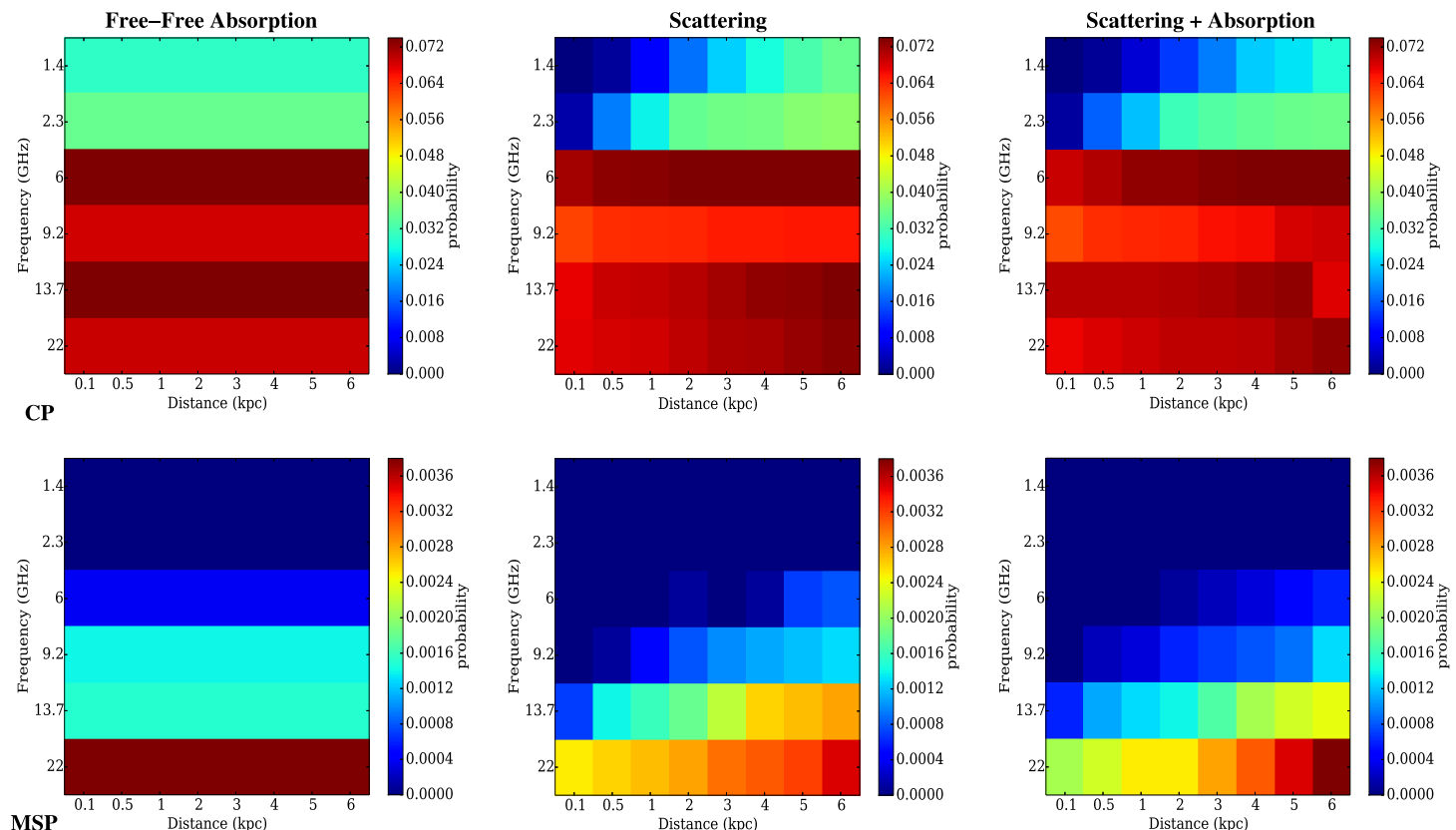

Figure 5.4: Probability of finding a pulsar in the GC as function of frequency and distance of the scattering screen from the GC in future GBT surveys assuming that the backend would be able to incorporate the whole bandwidth of each receiver. The columns from left to right are: free-free absorption, scattering, both scattering and absorption. The upper row is for CPs while the bottom one is for MSPs. 


\subsubsection{Probability of detection}

Finally, we computed a probability of detecting a single pulsar (CP and MSP) at the GC as a function of frequency and screen distance for each of the three scenarios (scattering, free-free absorption and both effects) by considering surveys of the GC with the GBT. We selected the GBT because it is the largest fully steerable single dish telescope where one can observe the GC for $\sim 7$ hours. We adopted the known parameters of GBT receivers from the GBT observing guide ${ }^{3}$ to compute the flux density limit at different frequencies for future GBT surveys (see Table 5.4). The sky contribution from the GC to the system temperature is significant and since the GC transits at an elevation of $\sim 21^{\circ}$, it was necessary to account for the changes in the system temperature, $T_{\text {sys }}$ at lower elevations. To do this we assumed, the system temperature of each receiver,

$$
T_{\mathrm{sys}}=T_{\mathrm{GC}}+T_{\mathrm{atm}}+T_{\mathrm{rec}}
$$

where, $T_{\mathrm{GC}}$ is the contribution of the $\mathrm{GC}, T_{\mathrm{atm}}$ is the contribution due to the atmosphere and $T_{\text {rec }}$ is the constant receiver temperature. $T_{\mathrm{GC}}$ is computed by taking the weighted average of $T_{\mathrm{GC}}(\nu)$ over the band of the receiver. To compute $T_{\mathrm{GC}}(\nu)$, we used the recent continuum maps of the $\mathrm{GC}$ at 1.4, 6 and $9.2 \mathrm{GHz}$ from Law et al. (2008). Using the calibrated maps, we used the flux density at the pixel

\footnotetext{
${ }^{3}$ https://science.nrao.edu/facilities/gbt/proposing/GBTpg.pdf
} 
corresponding to the GC to fit a power-law which led to a relationship

$$
T_{\mathrm{GC}}(\nu)=568\left(\frac{\nu}{\mathrm{GHz}}\right)^{-1.13} \mathrm{~K}
$$

For $T_{\text {atm }}$, we computed empirical relations between $T_{\text {atm }}$ and elevation for each receiver which made use of data from the GBT sensitivity calculator ${ }^{4}$. The $T_{\text {atm }}$ values we obtained ranged from $10-40 \mathrm{~K}$. Then, we computed the weighted average of $T_{\mathrm{atm}}$ over all hour angles of the source by taking into account the dependence of elevation with hour angle. The final $T_{\mathrm{sys}}$ is calculated by plugging in values for $T_{\mathrm{GC}}, T_{\mathrm{atm}}$ and $T_{\text {rec }}$ in Eq. 5.9. The final values of flux density sensitivities are given in Table 5.4 For multi-beam receivers, we assumed only a single beam. In these calculations, we are not assuming any coherent summing of multiple epochs. Using the flux densities computed in the simulation, we obtained flux density histograms of the synthesized population at different GBT frequencies and counted up the number of the pulsars above the flux density threshold of each survey. The required detection probability is simply the ratio of pulsars above each survey threshold to the total number of pulsars simulated.

\footnotetext{
${ }^{4}$ https://dss.gb.nrao.edu/calculator-ui/war/Calculator_ui.html
} 


\begin{tabular}{|c|c|c|c|c|c|c|c|c|}
\hline \multirow[t]{3}{*}{ Receiver } & \multirow{3}{*}{$\frac{\text { Central Frequency }}{(\mathrm{GHz})}$} & \multirow{3}{*}{$\frac{\text { Bandwidth }}{(\mathrm{MHz})}$} & \multirow{3}{*}{$\frac{10-\sigma \text { Sensitivity Limit }}{\mu \mathrm{Jy}}$} & \multirow{3}{*}{$\begin{array}{l}\text { VEGAS Limit } \\
\mu \mathrm{Jy}\end{array}$} & \multicolumn{4}{|c|}{ Detection probabilities expressed as percentages } \\
\hline & & & & & \multicolumn{2}{|c|}{ Future backends } & \multicolumn{2}{|r|}{ VEGAS } \\
\hline & & & & & $\mathrm{CP}$ & MSP & $\mathrm{CP}$ & MSP \\
\hline L-Band & 1.4 & 650 & 119 & 119 & $\leq 3.5$ & 0.0 & $\leq 3.5$ & 0.0 \\
\hline S-Band & 2.3 & 970 & 62.3 & 62.3 & $\leq 3.9$ & 0.0 & $\leq 3.9$ & 0.0 \\
\hline C-Band & 6 & 3800 & 12.2 & 20.3 & 8 & 0.08 & 5.3 & $\leq 0.04$ \\
\hline X-Band & 9.2 & 2400 & 11.3 & 16.3 & 7 & 0.14 & 5.2 & $0.05-0.09$ \\
\hline $\mathrm{Ku}$-Band & 13.7 & 3500 & 8.4 & 14.0 & 7.5 & $0.2-0.3$ & 5.3 & 0.1 \\
\hline KFPA & 22 & 8000 & 6.7 & 17.3 & 7.3 & $0.9-1.3$ & 0.4 & $0.1-0.2$ \\
\hline
\end{tabular}

Table 5.4: Table showing various parameters of the GBT receivers with corresponding survey limit for a future survey of the GC (see text for details). The difference in the survey sensitivities is due to different bandwidths assumed for VEGAS and future backends. The details for receivers are given in https://science.nrao.edu/facilities/gbt/facilities/gbt/proposing/ GBTpg.pdf. 
In 2012, a new backend was developed for the GBT. The VErsatile GBT Astronomical Spectrometer (VEGAS) is currently being used observations (Bussa \& VEGAS Development Team, 2012). The backend consists of eight different spectrometer banks and has a maximum total instantaneous bandwidth of $1250 \mathrm{MHz}$ for pulsar observations. VEGAS is expected to be the primary backend for pulsar astronomy and will replace the Green Bank Ultimate Pulsar Processing Instrument (GUPPI) (Ransom et al., 2009). Hence, in our analysis, we assume VEGAS to be the primary backend for future GBT pulsar surveys. Under these assumptions, we computed probability of detection for two scenarios: (a) the backend would be able to accommodate the entire bandwidth of each receiver; (b) using VEGAS as the backend in which case the bandwidth is limited to $1250 \mathrm{MHz}$ (column 4 in Table 5.4). The 2-D histograms for both the cases are shown in Fig. 5.4 and Fig. 5.5.

\subsubsection{Results}

Tables 5.3 and 5.4 clearly summarize our results from the analysis mentioned above. Table 5.3 shows the upper limits on the populations based on previous and future surveys for various models. The results point out that based on the null results from previous surveys, we can obtain an upper limit on the CP and MSP population in the GC and the results do not reject an existence of $\mathrm{CP}$ population in the GC. With the expected performance of SKA-MID and ngVLA, we would be able to probe a sizable population of GC pulsars which would give us much better constraints. The constraints on the pulsar population are less stringent as we 

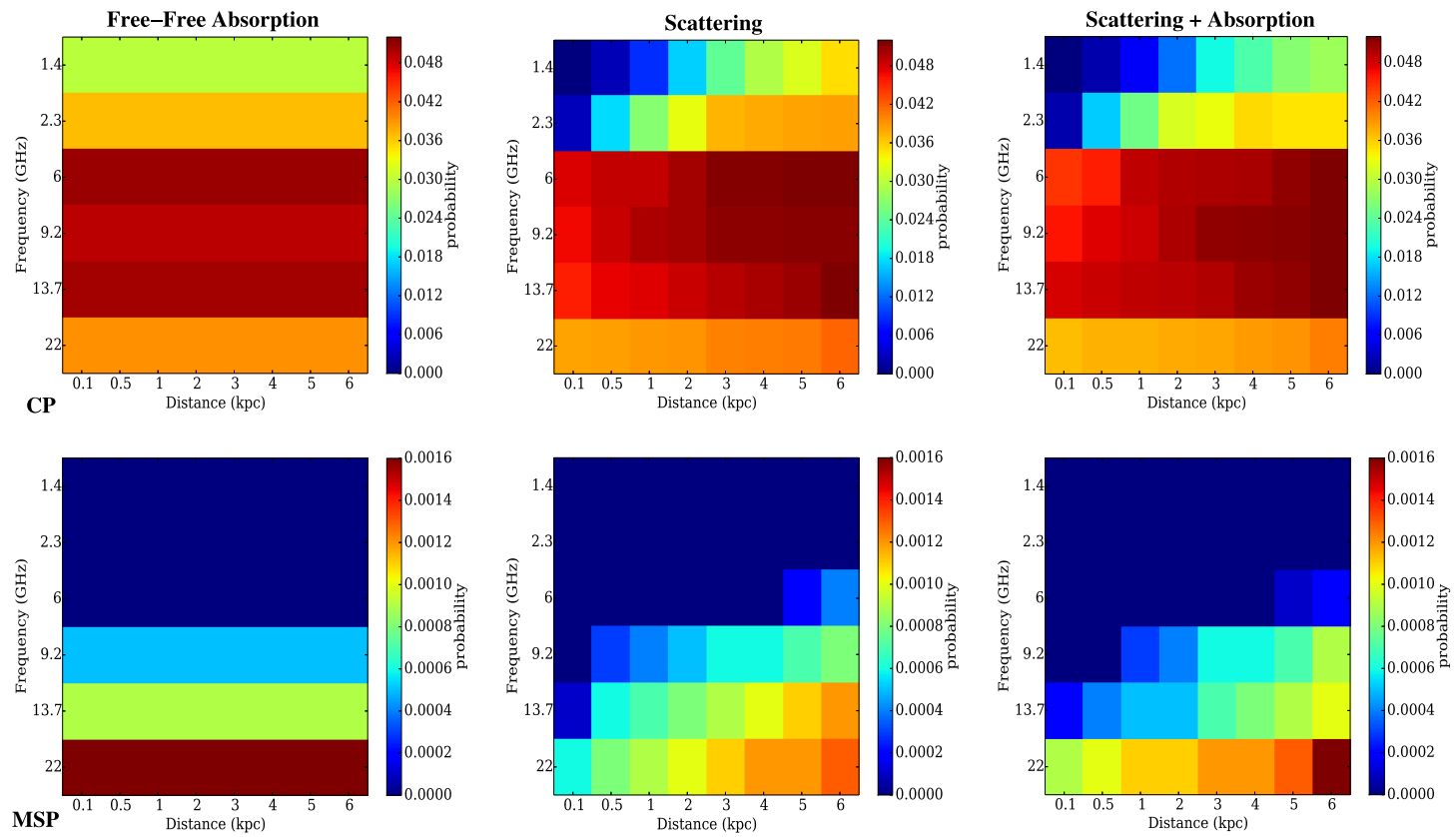

Figure 5.5: Probability of finding a pulsar in the GC as function of frequency and distance of the scattering screen from the GC. The probabilities have been computed for future GBT surveys and assuming VEGAS as the backend. The banding seen in the free-free absorption case is due to the different bandwidths of receivers on the GBT. 
include models for flux density mitigation as we would detect a lesser fraction of the existing population due to the effects of the ISM. Table 5.4 summarizes probabilities of finding one pulsar in a potential GBT survey. Results show that CPs have a better prospect of being detected than MSPs though the absolute probability is only as high as 0.07 . Moreover, Table 5.3 suggests that the allowed number of CPs is much lower than the number of MSPs. These upper limits show that a MSP dominated population is highly likely as shown in Macquart \& Kanekar (2015). The small number of predicted CPs would suggest that star formation is suppressed at the GC and that the existence of MSPs could be explained through capture of MSPs from globular cluster (Hooper \& Linden, 2016).

\subsection{Discussion}

Although the probability of detecting a single pulsar is greater than zero for higher frequencies, where scattering and absorption effects are negligible, the value itself is small. This can be attributed to the distance of the GC where the flux densities of pulsars in the GC would be so small that even without assuming any attenuation of the flux density, we have been able to probe only a small fraction of the population. Irrespective of the dominance of sub populations in the $\mathrm{GC}(\mathrm{CP}$ or MSP), the faintness of these sources due to the distance of the GC makes it difficult to detect them. This is clearly indicated by Fig. 5.2 where the survey sensitivity limit only encloses $0-2 \%$ of the total simulated population of CPs and $0 \%$ of the total MSP population for the baseline model. This shows that we need deeper searches of 
the GC in the future even if the environment does not play a role in affecting pulsar flux densities. Our results predict $\sim 52$ CPs beaming towards us within 1 pc of the GC, which is a tighter constraint on the total CP population compared to the 200 CPs predicted in Chennamangalam \& Lorimer (2014). Chennamangalam \& Lorimer (2014) take into account the magnetar population as a magnetar fraction in the GC and their results suggest previous surveys were not sensitive to existing pulsar population in the GC. Dexter \& O'Leary (2014) suggest that given the absence of hyperstrong scattering and lack of pulsar detections, there might be an intrinsic deficit of pulsars in the GC though our simulations suggest our radio surveys have not been sensitive enough to detect any pulsars in the GC. The detection of one magnetar hints at a preference to creation of magnetars in the GC. Future SKA and ngVLA surveys will be able to answer these questions.

Figs. 5.4 and 5.5 show the probability of detection for different frequencies and screen distances for MSPs and CPs. The figures show that free-free absorption has negligible effect on the flux density mitigation beyond frequencies of $1 \mathrm{GHz}$ due to negligible optical depths at higher frequencies. Hence, the probability of detection is solely dependent on the bandwidth of the telescope receivers. On the other hand, scattering plays an important role in reducing flux density from pulsars. Scattering transitions from strong scattering to weak scattering regime as the distance of the screen from the GC increases. Hence, one would expect to have maximum yield from the GC survey when the screen is far enough from the GC and the survey is at a high frequency. These aforementioned effects help us in constraining the optimum frequency for future GC surveys. Note that the optimum frequency largely depends 
on the bandwidth of the survey if it is backend limited. The 2-D histograms also suggest that the optimum frequency for future GBT surveys is as high as $9 \mathrm{GHz}$ for CPs and $22 \mathrm{GHz}$ for MSPs for strong and weak scattering cases if we assume the backends can cover the whole bandwidth of the receiver. On the other hand, if we consider VEGAS as the backend for future surveys, we obtain an optimum frequency of $\sim 9 \mathrm{GHz}$ for CPs for both the strong and the weak scattering case. For MSPs, the optimum frequency is $22 \mathrm{GHz}$ for the weak and strong scattering case with VEGAS. Since we are interested in finding CPs and MSPs, based on these results, we propose that the optimal range of frequencies for future GBT surveys is $9-14 \mathrm{GHz}$. We also note that future surveys of the GC in the range of $1.4-6 \mathrm{GHz}$ will not be able to detect MSPs the faintness of the sources and scattering affects MSPs significantly at lower frequencies. In any case, we have to go to higher frequencies (> $9 \mathrm{GHz})$ to detect any pulsars in the GC in single observational tracks.

The results suggest that it would be more difficult to detect MSPs than CPs given the lower radio luminosities and the effect scattering has on their radio flux densities. We cannot favor any population at the moment because the analysis suggests that previous surveys have not been sensitive to any of the populations so far, even without factoring in the sources of flux density mitigation. Our conclusions differ from Macquart \& Kanekar (2015), which can be attributed to the fact that the population used in that paper is the actual pulsar population, which might have an inherent selection bias in the pseudo-luminosity function of the source population as only the brightest pulsars have been detected by current radio telescopes. Hence, we sample only the tail of the underlying pseudo-luminosity distribution of pulsars, 
which can lead to different inferences about the source population. On the other hand, we have considered a synthetic population of pulsars in the GC, assuming an underlying pseudo-luminosity function, which properly accounts for this selection bias.

We also report upper limits on the CP and MSP population which are more stringent compared to the ones reported in Chennamangalam \& Lorimer (2014). Recent results are suggesting that scattering does not play an important role in the attenuation of flux densities towards the GC. This is an important result for future surveys of the GC. If the weak scattering scenario is true, then Fig. 5.2 suggests that deeper searches of the GC without going to higher and higher frequencies would result in more detections of pulsars. Future telescopes like the SKA and next generation Very Large Array (ngVLA) (Hughes et al., 2015) will provide a great opportunity to search for radio pulsars in the GC. These surveys are expected to detect significant fraction of the pulsar population in the inner Galaxy. Future high frequency radio surveys with highly sensitive radio telescopes will help in resolving the pulsar problem in the GC.

\subsection{Conclusions}

In summary, from an analysis of the current observational constraints of the pulsar population in the GC, our main conclusions are as follows: (i) the null results from previous surveys are not surprising, given that current surveys have only probed $\sim 3 \%$ of the total $\mathrm{CP}$ population and $0 \%$ of the MSP population; (ii) upper limits 
on the CP and MSP population for various models constrain the population of pulsars beaming towards us to be $<52$ CPs and $<10000$ MSPs; (iii) we predict the existence of CPs, along with MSPs in the GC though their numbers are expected to much smaller; (iv) a future GC survey with the GBT would have greater prospects of detecting CPs compared to MSPs. We find that the optimum frequency of a GBT survey would be 9-14 GHz; (v) a future survey with SKA-MID and ngVLA would probe a sizable population of the pulsar population in the GC. 


\section{Chapter 6}

\section{Conclusions}

By studying pulsars and FRBs, we can probe the physical properties and structure of the ISM and the IGM. Here, I briefly summarize my conclusions from the work presented in this dissertation.

\subsection{GPS pulsars}

By modeling spectra of GPS pulsars, one can put observational constraints on the geometry, density and the temperature of the absorber along the line of sight. We were able to constrain the linear size, the electron density and temperature for the absorber in a sample of GPS pulsars. Since there is a degeneracy between the electron temperature and emission measure for the absorber, it is difficult to pinpoint the exact nature of the astrophysical absorber. We made our best estimate for the source of absorption based on simple physical arguments by comparing our derived values to the observed values of known absorbers. A larger sample of GPS pulsars will certainly help in gaining insights into the physical properties of the absorbing ISM in the Galaxy. Such studies will also give astronomers information to optimize targeted pulsar surveys in the future to look for young neutron stars within dense, ionized SNR shells. On a similar note, pulsars that lie within or beyond large HII regions would be difficult to detect due to significant absorption along the line of 
sight. If the pulsar velocity in these regions is higher than the sound velocity of the medium, they will form bowshock nebulae that will be visible at optical wavelengths. We plan to search for such optical signatures in HII regions with an aim to find GPS pulsars in these dense regions.

\subsection{Simultaneous radio and X-ray observations of PSR B0611+22}

We studied PSR B0611+22 in a multi-wavelength campaign that revealed peculiar emission behavior of the pulsar at different radio frequencies. The pulsar showed mode changes that were anti-correlated over different radio frequencies. Such emission variability in frequency and time cannot be explained in the standard framework. We plan on follow-up radio observations over a wider range of frequencies aimed at disentangling the emission behavior of the pulsar. Moreover, the location of the pulsar in the vicinity of IC 443 (though the association has been ruled out) provided us an opportunity to study the flux density spectrum. The flux density spectrum shows an interesting trend, which looks like an absorbed power-law at $320 \mathrm{MHz}$, only to turn back to a standard power-law at $150 \mathrm{MHz}$. This interesting spectral behavior has implications on the intrinsic mechanism of the pulsar. The phase shift of the bright phase suggests that different emission components have different spectral indices, leading to a switch at a certain frequencies. Other postulates include scintillation of radio waves within the magnetopshere causing brightening and dimming at different frequencies. The study of a larger sample of pulsars showing bursty emission would help in revealing the true nature of the emission physics 
in these pulsars.

\subsection{Detecting FRBs at decametric wavelengths}

Using simple assumptions, we have shown that future low frequency (400$800 \mathrm{MHz}$ ) surveys will prove extremely useful in finding FRBs. Even for models involving both absorption and scattering, we predict that CHIME and HIRAX will detect $\sim 30$ FRBs per day. Though the results are promising, one should note that the assumptions we made might be too simple and we should be careful about them. This gives us an opportunity to work on the caveats discussed in Chapter 4 for the

future. This involves using different luminosity distributions for FRBs instead of a standard candle model, fluence dependent rates and experimental curves of $T_{\text {sys }}$ and $G$ for CHIME and HIRAX to calculate FRB yields. We believe that we should have a reasonably large sample of FRBs to study in the near future. Furthermore, we observed peculiar behavior of the peak flux at high redshifts when the spectrum of the FRB is absorbed. This suggests that for absorption models, low frequency surveys will be able to detect FRBs at higher redshifts $(\mathrm{z} \approx 1)$. Future surveys with LOFAR and Long Wavelength Array will be able to constrain this model.

\subsection{GC pulsar population}

The detection of pulsars in the GC is important for studying the supermassive black hole in our Galaxy. In this study, we attempt to tackle the problem of pulsar population in the GC. We find that previous surveys have been insensitive to the 
existing population. We go on to find probability of detection for future GBT surveys assuming free-free absorption and scattering and found 9-14 GHz to be the optimal band to survey the GC for pulsars. Moreover, we obtained conservative upper limits on the population of CPs and MSPs that are more constraining compared to previous results. As a next step, we have an upcoming observation campaign to search the GC with the GBT with a promise to find pulsars in the GC. We plan to observe the $\mathrm{GC}$ at $9 \mathrm{GHz}$ in three sessions spanning 6.5 hours, which is close to maximum time the GC is observable from Green Bank. We will use the pulsar mode of the VEGAS backend, giving us $1250 \mathrm{MHz}$ of usable bandwidth at $9 \mathrm{GHz}$.

The research presented in this dissertation shows that the study of radio transients and the ISM complement each other. We can use transient sources to probe the intervening medium and gain insights into the physical properties of the material between the source and the observer. At the same time, we can use the known properties of the ISM and the assumed effects it would have on potential transient signals to predict the source population, the spectral indices and detection rates of these sources. 


\section{Appendix A}

\section{Derivation of optical depth}

Here, we derive the formula for optical depth of a dense, ionized region under the assumption of LTE. In LTE, we assume that the temperature of the plasma is constant and the energy levels are populated such that there is a balance between emission and absorption. Moreover, we assume that the particles follow a Maxwellian distribution for velocities.

From Condon \& Ransom (2016), the pulse energy $W$ radiated by a single electron due to an interaction with an ion,

$$
W=\frac{\pi Z^{2} e^{6}}{4 c^{3} m_{e}^{2}}\left(\frac{1}{b^{3} v}\right)
$$

where $Z$ is the atomic number of the species involved, $b$ is the impact parameter and $v$ is the velocity. The spectrum of such a radiation is mostly flat at frequencies $\nu \ll \nu_{\max }=(v / 2 \pi b)$ and falls rapidly at higher frequencies.

The spectrum of radio emission from an HII region depends on the velocity distribution and impact factors. If an electron moves with a velocity $v$ in time $t$ in a cylinder with radius $b$ and width $d b$, the number of electrons passing any ion per unit time with impact parameter $b$ to $b+d b$ and speed range $v$ to $v+d v$ is

$$
n=n_{e}(2 \pi b d b) v f(v) d v
$$




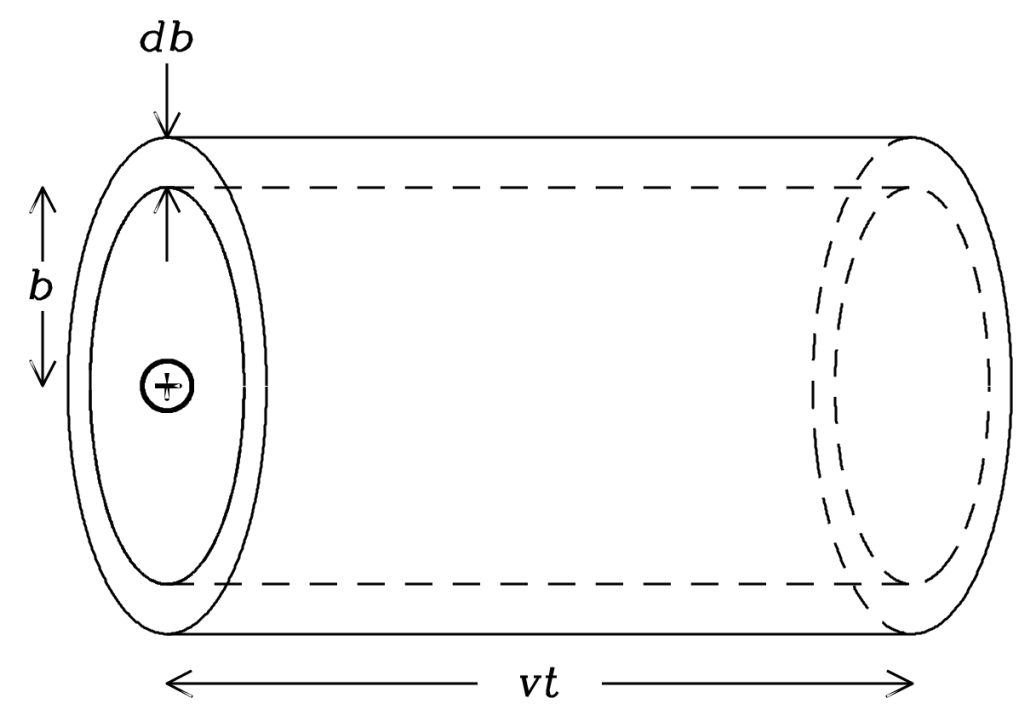

Figure A.1: Diagram showing the probability of collision for a given impact parameter.

where $f(v)$ is the velocity distribution of electrons. Then, the number of collisions of electrons per unit volume per unit time,

$$
\dot{n}_{e}=(2 \pi b) v f(v) n_{e} n_{i} .
$$

Hence, the total power emitted by electrons,

$$
4 \pi j_{\nu}=\int_{0}^{\infty} \int_{0}^{\infty} W_{\nu(v, b)} \dot{n}_{e} d b d v
$$

where $j_{\nu}$ is the spectral power emitted isotropically per unit volume.

Since $b$ cannot have infinite length, we need to define a range of impact parameters based on known knowledge of the ISM and the physical limits it imposes on $b$. In local thermodynamic equilibrium, $f(v)$ follows a Maxwellian distribution 
given by,

$$
f(v)=\frac{4 v^{2}}{\pi}\left(\frac{m_{e}}{2 k_{b} T}\right)^{1.5} \exp \left(-\frac{m_{e} v^{2}}{2 k_{b} T}\right) .
$$

After plugging in Eqs A.5, A.2 and A.3 in Eq. A.4 we can obtain the the free-free emission coefficient of the emissivity,

$$
j_{\nu}=\frac{\pi^{2} Z^{2} e^{6} n_{e} n_{i}}{4 c^{3} m_{e}}\left(\frac{2 m_{e}}{\pi k T}\right)^{0.5} \ln \left(\frac{\mathrm{b}_{\max }}{\mathrm{b}_{\min }}\right) .
$$

The $b_{\min }$ comes from the limit of momentum transfer between the electron and the ion during a free-free interaction. The maximum momentum transfer cannot be more than twice the momentum of the electron. Hence, the impact parameter cannot be smaller than,

$$
b_{\min } \approx \frac{Z e^{2}}{m_{e} v^{2}} .
$$

A quantum mechanical limit can be obtained from the uncertainty principle ( $\Delta x \Delta p \sim$ ћ) such that,

$$
b_{\min }=\frac{\hbar}{m_{e} v} .
$$

This lower limit is much smaller than the classical one for HII regions and Pulsar Wind Nebulae (PWNe) (Gaensler \& Slane, 2006, and references therein) and hence can be ignored.

The maximum limit comes from the fact that free electrons can interact with ions however they want and rearrange themselves to screen the electric field as electrostatic force dominates over gravity at small length scales. The characteristic 
length of screening of the electric field is given by the Debye length,

$$
\lambda_{D}=\left(\frac{k T}{4 \pi n_{e} e^{2}}\right)
$$

Another way to compute a maximum limit on the impact parameter is by assuming the largest value of $b$ that can emit significant amout of power in radio. Thus,

$$
b_{\max } \approx \frac{v}{2 \pi \nu}
$$

Hence, plugging in standard values for an electron and assuming Maxwelllian distribution for $f(v)$ in Eq A.6 we get,

$$
\ln \left(\frac{\mathrm{b}_{\max }}{\mathrm{b}_{\min }}\right) \simeq 12
$$

Since HII regions are in LTE, we can obtain the coefficient of free-free absorption by using Kirchoff's law (Condon \& Ransom, 2016),

$$
\kappa=\frac{j_{\nu}}{B_{\nu}(T)} \approx \frac{j_{\nu} c^{2}}{2 k T \nu^{2}}
$$

Plugging in $j_{\nu}$ in Eq. A.12 and we can write the absorption coefficient,

$$
\kappa=\frac{1}{\nu^{2} T^{3 / 2}}\left[\frac{Z^{2} e^{6}}{c} n_{e} n_{i} \frac{1}{\sqrt{\pi\left(m_{e} k\right)^{3}}}\right] \frac{\pi^{2}}{4} \ln \left(\frac{\mathrm{b}_{\max }}{\mathrm{b}_{\min }}\right) .
$$

If we assume that the plasma is macroscopically neutral then to a good approxima- 
tion, $n_{e} \approx n_{i}$. Now, we can define the optical depth of an ionized region,

$$
\tau=-\int_{0}^{l} \kappa d s \propto \int \frac{n_{e} n_{i}}{\nu^{2} T^{3 / 2}} d s \approx \int \frac{n_{e}^{2}}{\nu^{2} T^{3 / 2}} .
$$

For convenience, we can define the "Emission Measure (EM)",

$$
\mathrm{EM}=\int n_{e}^{2} d s
$$

i.e., the integrated electron density squared along the line of sight. Plugging in Eq. A.15 in Eq. A.14 and the result we get is similar to the one obtained from detailed mathematical calculations in (Oster, 1961) where the optical depth,

$$
\tau \simeq 3.014 \times 10^{-2}\left(\frac{T}{\mathrm{~K}}\right)^{-1.5}\left(\frac{\nu}{\mathrm{GHz}}\right)^{-2}\left(\frac{\mathrm{EM}}{\mathrm{pc} \mathrm{cm}^{-6}}\right)\left\langle g_{f f}\right\rangle,
$$

and the Gaunt factor,

$$
\left\langle g_{f f}\right\rangle \approx \ln \left[4.955 \times 10^{-2}\left(\frac{\nu}{\mathrm{GHz}}\right)^{-1}\right]+1.5 \ln \left(\frac{T}{\mathrm{~K}}\right) .
$$

The Gaunt factor is the quantum mechanical correction to the free-free absorption in a thermalized plasma. In general, obtaining a value of the Gaunt factor can be non-trivial for HII regions hence we can represent $\left\langle g_{f f}\right\rangle \simeq \alpha T^{\beta} \nu^{\gamma}$ and plug that in Eq. A.16 and a simpler equation (Altenhoff et al., 1960),

$$
\tau \approx 0.082 a(T, \nu)\left(\frac{T}{\mathrm{~K}}\right)^{-1.35}\left(\frac{\mathrm{EM}}{\mathrm{cm}^{-6} \mathrm{pc}}\right)\left(\frac{\nu}{\mathrm{GHz}}\right)^{-2.1},
$$



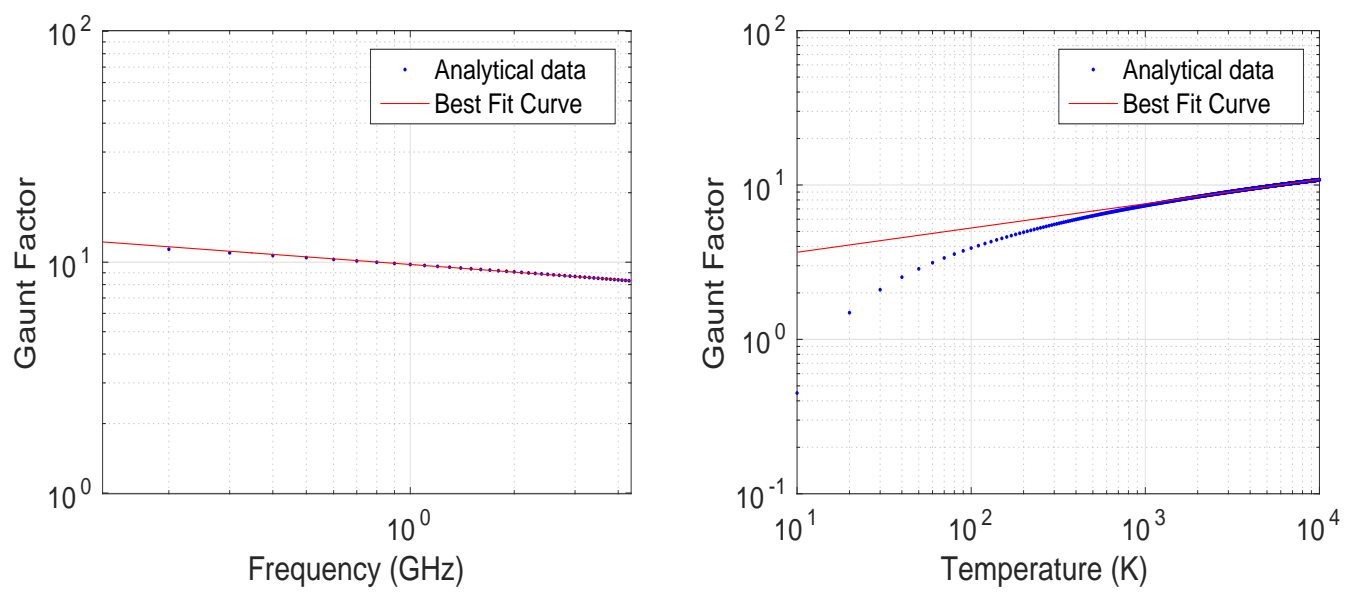

Figure A.2: Figure showing curve for Gaunt Factor versus frequency (Left) and Gaunt factor versus temperature (Right) alongwith the best fit power-law model. We obtained $\alpha=-0.11$ and $\beta=0.16$ with a reduced $\chi^{2}=0.99$.

can be derived. To illustrate this, we have shown figure. A.2 where we fit for $\alpha$ and $\beta$ by keeping the other fixed and we obtained the expected values of the exponents to give us the final equation. Here $a(T, \nu)$ is a correction factor because of the approximation. For the temperatures of HII regions/PWNe, we can assume $a \approx 1$ 


\section{Appendix B}

\section{Calculation of scattering efficiency}

Here, we describe the method to calculate the reduction in flux due to scattering. Since pulsar surveys make use of harmonic summing to increase the signal to noise of the detection in the Fourier domain, for each of previous and future survey, we find the optimum number of harmonics to be summed. For any survey, we follow the terminology in Cordes \& Chernoff (1997) and define the "pulsed fraction"

$$
\eta_{p}=\sum_{0}^{N_{h}} \frac{R_{l}}{\sqrt{N_{h}}},
$$

where $N_{h}$ is the number of harmonics to be summed and

$$
R_{l}=\frac{S(l)}{S(0)}
$$

is the ratio of the amplitude of the $l^{\text {th }}$ harmonic and the amplitude of the DC component in the Fourier domain. For this analysis, we assume a Gaussian pulse characterized by

$$
f_{1}(t)=\frac{1}{\sqrt{2 \pi} \sigma} \exp \left[\frac{-t^{2}}{2 \sigma^{2}}\right],
$$

where $\sigma$ is the standard deviation and in our case, $t$ is time running over one pulse period, $P$. For the scattered case, we convolve the Gaussian with a one-sided exponential function with a mean of $\tau_{s}$. This results in a modified pulse profile described 
by

$$
f_{2}(t)=\frac{\lambda}{2} \exp \left(\sigma^{2} \lambda-2 t\right) \operatorname{erfc}\left(\sigma^{2} \lambda-t\right)
$$

where $\lambda=1 / \tau_{s}$ and $\sigma$ is the standard deviation of the Gaussian distribution and the complimentary error function

$$
\operatorname{erfc}(x)=\frac{2}{\sqrt{\pi}} \int_{x}^{\infty} e^{-y^{2}} d y .
$$

The scattering broadening function in the time domain is given by,

$$
f_{s c a}(t)=\exp \left(-\frac{t}{\tau_{s}}\right) .
$$

In Fourier space, where the frequency of the $l^{\text {th }}$ harmonic $k=l / P$, the Gaussian pulse transforms to

$$
S_{\text {Gauss }}(k)=\frac{1}{\sqrt{2 \pi}} \exp \left(-\frac{\sigma^{2} k^{2}}{2}\right)
$$

and the scatter broadening function transforms to

$$
S_{\mathrm{sca}}(k)=\frac{1}{\left(k^{2} \tau_{s}^{2}+1\right)}
$$

The resulting Fourier components are then

$$
S(k)=S_{\mathrm{Gauss}}(k) \cdot S_{\mathrm{sca}}(k) .
$$



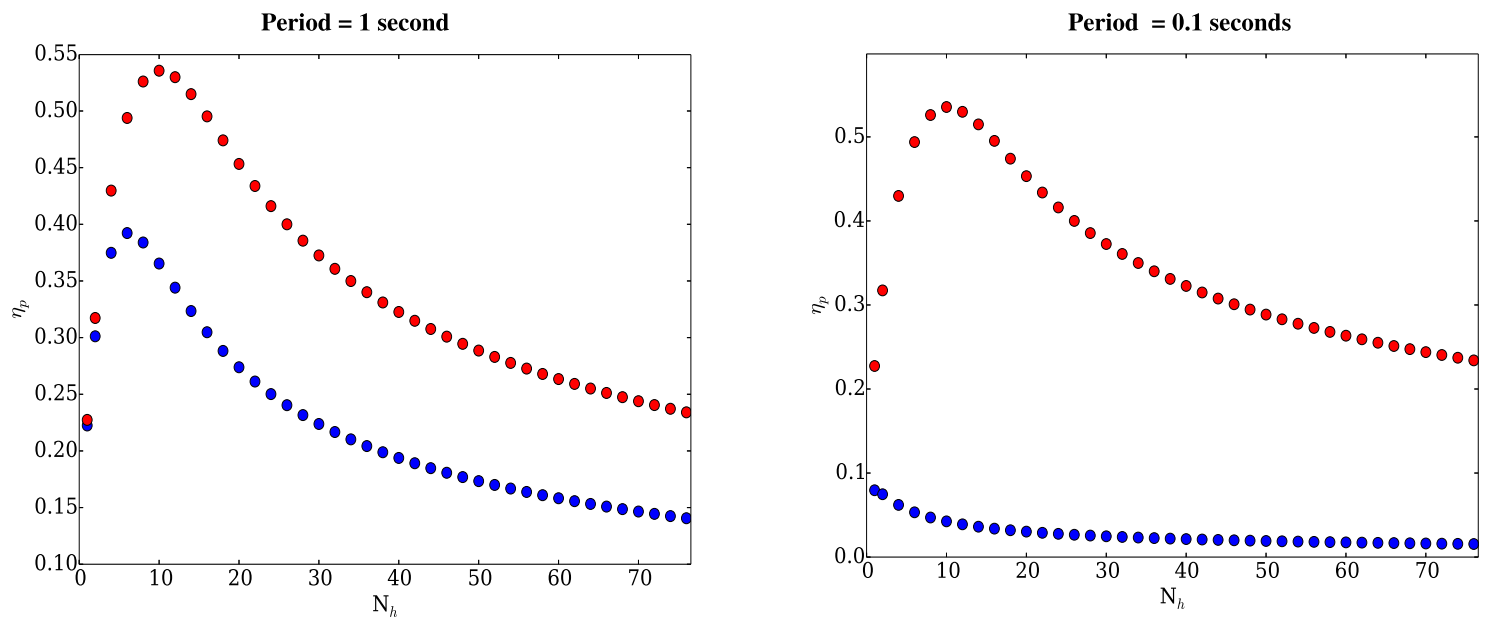

Figure B.1: Pulsed fraction versus number of harmonics summed for a Gaussian pulse (red dots) and a scattered pulse (blue dots). We assume a constant duty cycle of $5 \%$. We can see that smaller periods are severely affected by scattering.

The functions $f_{1}(t)$ and $f_{2}(t)$ reported here are already normalized to make sure that the area under the pulse within one pulse period is the same for both functions. After normalizing the pulse from both scenarios, we computed the Fourier transform for the standard and scattered pulse.

Then, we obtained the optimal number of harmonics to be summed and computed the pulsed fraction using Eq. B.1. The optimum number of harmonics to be summed will be the value $N_{h}$ for which Eq. B.1 is maximized. Figure. B.1 shows one such result for a strong scattering scenario for CPs for a fixed duty cycle. In the case of strong scattering, the value of $N_{h}$ is lower and the maximum value of the pulsed fraction is significantly lower than the unscattered case. This means that the sensitivity of the survey reduces by a factor of the ratio of the two pulsed fractions,

$$
\mathcal{S}(\nu)=\frac{\eta_{\mathrm{p}, \mathrm{g}}}{\eta_{\mathrm{p}, \mathrm{sca}}}
$$




\section{Bibliography}

Abdo A. A., Ajello M., Allafort A., Baldini L., Ballet J., Barbiellini G., Baring M. G., Bastieri D., Belfiore A., Bellazzini R., et al. 2013, ApJS, 208, 17

Alpar M. A., Cheng A. F., Ruderman M. A., Shaham J., 1982, Nature, 300, 728

Altenhoff W. J., Mezger P. G., Wendker H., Westerhout G., 1960, Veröff. Sternewärte, Bonn, 59, 48

Arons J., Scharlemann E. T., 1979, ApJ, 231, 854

Arzoumanian Z., Brazier A., Burke-Spolaor S., Chamberlin S. J., Chatterjee S., Christy B., Cordes J. M., Cornish N. J., NANOGrav Collaboration 2016, ApJ, 821,13

Arzoumanian Z., Nice D. J., Taylor J. H., Thorsett S. E., 1994, ApJ, 422, 671

Backer D. C., 1970a, Nature, 228, 752

Backer D. C., 1970b, Nature, 227, 692

Backer D. C., 1970c, Nature, 228, 1297

Backer D. C., 1970d, Nature, 228, 42

Bailes M., Johnston S., Bell J. F., Lorimer D. R., Stappers B. W., Manchester R. N., Lyne A. G., Nicastro L., Gaensler B. M., 1997, ApJ, 481, 386

Bartel N., Sieber W., Graham D. A., 1980, A\&A, 87, 282 
Bartel N. \& Hankins T. H., 1982, ApJL, 254, L35

Bates S. D., Johnston S., Lorimer D. R., Kramer M., Possenti A., Burgay M., Stappers B., Keith M. J., Lyne A., Bailes M., McLaughlin M. A., O’Brien J. T., Hobbs G., 2011, MNRAS, 411, 1575

Bates S. D., Lorimer D. R., Rane A., Swiggum J., 2014, MNRAS, 439, 2893

Bates S. D., Lorimer D. R., Verbiest J. P. W., 2013, MNRAS, 431, 1352

Becker W., Truemper J., 1997, A\&A, 326, 682

Berkhuijsen E. M., Müller P., 2008, A\&A, 490, 179

Bhat N. D. R., Cordes J. M., Camilo F., Nice D. J., Lorimer D. R., 2004, ApJ, 605, 759

Bhat N. D. R., Gupta Y., Kramer M., Karastergiou A., Lyne A. G., Johnston S., 2007, A\&A, 462, 257

Bhattacharya D., Wijers R. A. M. J., Hartman J. W., Verbunt F., 1992, A\&A, 254, 198

Biggs J. D., 1992, ApJ, 394, 574

Bilous A., Kondratiev V., Kramer M., Keane E., Hessels J., Stappers B., Malofeev V., Sobey C. e. a., 2015, ArXiv e-prints

Bocchino F., Bykov A. M., 2000, A\&A, 362, L29 
Bower G. C., Deller A., Demorest P., Brunthaler A., Eatough R., Falcke H., Kramer M., Lee K. J., Spitler L., 2014, ApJL, 780, L2

Brederode L. R., van den Heever L., Esterhuyse W., Jonas J. L., 2016, in Society of Photo-Optical Instrumentation Engineers (SPIE) Conference Series Vol. 9906, MeerKAT: a project status report. p. 990625

Brisken W. F., Benson J. M., Goss W. M., Thorsett S. E., 2002, ApJ, 571, 906

Burgay M., Bailes M., Bates S. D., Bhat N. D. R., Burke-Spolaor S., Champion D. J., Coster P., D'Amico N., et al. 2013, MNRAS, 433, 259

Burke B. F., Graham-Smith F., 2014, An Introduction to Radio Astronomy. Cambridge University Press

Burke-Spolaor S., Bailes M., Ekers R., Macquart J.-P., Crawford III F., 2011, ApJ, 727,18

Bussa S., VEGAS Development Team 2012, in American Astronomical Society Meeting Abstracts \#219 Vol. 219 of American Astronomical Society Meeting Abstracts, VEGAS: VErsatile GBT Astronomical Spectrometer. p. 446.10

Bychkov K. V., Lebedev V. S., 1979, A\&A, 80, 167

Caleb M., Flynn C., Bailes M., Barr E. D., Bateman T., Bhandari S., CampbellWilson D., Farah W., et al. 2017, ArXiv e-prints

Caleb M., Flynn C., Bailes M., Barr E. D., Bateman T., Bhandari S., CampbellWilson D., Green A. J., et al. 2016, MNRAS, 458, 718 
Caleb M., Flynn C., Bailes M., Barr E. D., Hunstead R. W., Keane E. F., Ravi V., van Straten W., 2016, MNRAS, 458, 708

Camilo F., Ransom S. M., Chatterjee S., Johnston S., Demorest P., 2012, ApJ, 746, 63

Camilo F., Reynolds J., Johnston S., Halpern J. P., Ransom S. M., van Straten W., 2007, ApJL, 659, L37

Carilli C. L., McKinnon M., Ott J., Beasley A., Isella A., Murphy E., Leroy A., Casey C., et al. 2015, ArXiv e-prints 1510.06438

Champion D. J., Petroff E., Kramer M., Keith M. J., Bailes M., Barr E. D., Bates S. D., Bhat N. D. R., et al. 2016, MNRAS

Chatterjee S., Law C. J., Wharton R. S., Burke-Spolaor S., Hessels J. W. T., Bower G. C., Cordes J. M., Tendulkar S. P., Bassa C. G., et al. 2017, Nature, 541, 58

Chawla P., Kaspi V. M., Josephy A., Rajwade K. M., Lorimer D. R., Archibald A. M., DeCesar M. E., Hessels J. W. T., et al. 2017, ArXiv e-prints 1701.07457

Cheng K. S., Ho C., Ruderman M., 1986, Astrophys. J., 300, 500

Chennamangalam J., Lorimer D. R., 2014, MNRAS, 440, L86

Chevalier R. A., 1998, ApJ, 499, 810

Churchwell E., 2002, ARA\&A, 40, 27

Condon J., Ransom S., 2016, Essential Radio Astronomy. Princeton University Press 
Connor L., Lin H.-H., Masui K., Oppermann N., Pen U.-L., Peterson J. B., Roman A., Sievers J., 2016, MNRAS, 460, 1054

Contopoulos I., Spitkovsky A., 2006, ApJ, 643, 1139

Cordes J. M., Bhat N. D. R., Hankins T. H., McLaughlin M. A., Kern J., 2004, ApJ, 612, 375

Cordes J. M., Chernoff D. F., 1997, ApJ, 482, 971

Cordes J. M., Lazio T. J. W., 1997, ApJ, 475, 557

Cordes J. M., Lazio T. J. W., 2002a, ArXiv e-prints

Cordes J. M., Lazio T. J. W., 2002b, ArXiv Astrophysics e-prints

Cordes J. M., Wasserman I., 2016, MNRAS, 457, 232

Cordes J. M., Wharton R. S., Spitler L. G., Chatterjee S., Wasserman I., 2016, ArXiv e-prints 1605.05890

Crawford F., Rane A., Tran L., Rolph K., Lorimer D. R., Ridley J. P., 2016, MNRAS

Davies J. G., Lyne A. G., Seiradakis J. H., 1972, Nature, 240, 229

Dembska M., Kijak J., Jessner A., Lewandowski W., Bhattacharyya B., Gupta Y., 2014, MNRAS, 445, 3105

Deneva I. S., 2010, PhD thesis, Cornell University

Deneva J. S., Stovall K., McLaughlin M. A., Bagchi M., Bates S. D., Freire P. C. C., Martinez J. G., Jenet F., Garver-Daniels N., 2016, ApJ, 821, 10 
Dexter J., O’Leary R. M., 2014, ApJL, 783, L7

Dionysopoulou K., Alic D., Palenzuela C., Rezzolla L., Giacomazzo B., 2013, Phys. Rev. D, 88, 044020

Duin R. M., van der Laan H., 1975, A\&A, 40, 111

Dulk G. A., Slee O. B., 1975, ApJ, 199, 61

Dyks J., Rudak B., Harding A. K., 2004, ApJ, 607, 939

Eatough R., Karuppusamy R., Kramer M., Klein B., Champion D., Kraus A., Keane E., Bassa C., Lyne A., Lazarus P., Verbiest J., Freire P., Brunthaler A., Falcke H., 2013, The Astronomer's Telegram, 5040

Edwards R. T., Bailes M., van Straten W., Britton M. C., 2001, MNRAS, 326, 358

Falcke H., Rezzolla L., 2014, A\&A, 562, A137

Faucher-Giguère C.-A., Kaspi V. M., 2006, ApJ, 643, 332

Fesen R. A., 1984, ApJ, 281, 658

Gaensler B. M., Slane P. O., 2006, ARA\&A, 44, 17

Gajjar V., Joshi B. C., Kramer M., 2012, MNRAS, 424, 1197

Gajjar V., Joshi B. C., Wright G., 2014, MNRAS, 439, 221

Gangadhara R. T., Gupta Y., 2001, Astrophys. J., 555, 31

Geyer M., Karastergiou A., 2016, MNRAS, 462, 2587 
Ghisellini G., 2017, MNRAS, 465, L30

Gil J., Melikidze G. I., 2002, ApJ, 577, 909

Gillessen S., Genzel R., Fritz T. K., Quataert E., Alig C., Burkert A., Cuadra J., Eisenhauer F., Pfuhl O., Dodds-Eden K., Gammie C. F., Ott T., 2012, Nature, 481,51

Goldreich P., Julian W. H., 1969, Astrophys. J., 157, 869

Graham-Smith F., 2003, Reports on Progress in Physics, 66, 173

Gupta Y., Gil J., Kijak J., Sendyk M., 2004, A\&A, 426, 229

Gupta Y., Rickett B. J., Lyne A. G., 1994, MNRAS, 269, 1035

Hamaker J. P., 2006, A\& A, 456, 395

Hamil O., Stone J. R., Urbanec M., Urbancová G., 2015, Phys. Rev. D, 91, 063007

Hankins T. H., Kern J. S., Weatherall J. C., Eilek J. A., 2003, Nature, 422, 141

Hanson R. B., 1979, MNRAS, 186, 357

Harding A. K., Muslimov A. G., 2002, ApJ, 568, 862

Haslam C. G. T., Salter C. J., Stoffel H., Wilson W. E., 1982, AAPS, 47, 1

Hermsen W., Hessels J. W. T., Kuiper L., van Leeuwen J., Mitra D., de Plaa J., Rankin J. M., Stappers B. W., et al. 2013, Science, 339, 436 
Hessels J. W. T., Ransom S. M., Stairs I. H., Freire P. C. C., Kaspi V. M., Camilo F., 2006, Science, 311, 1901

Hewish A., Bell S. J., Pilkington J. D. H., Scott P. F., Collins R. A., 1968, Nature, 217,709

Hill L. E., 1972, MNRAS, 157, 419

Hobbs G., Lorimer D. R., Lyne A. G., Kramer M., 2005, MNRAS, 360, 974

Hooper D., Linden T., 2016, JCAP, 8, 018

Hughes A. M., Beasley A., Carilli C., 2015, IAU General Assembly, 22, 2255106

Inoue S., 2004, MNRAS, 348, 999

Jackson J. D., 1962, Classical Electrodynamics. Wiley

Johnston S., Karastergiou A., Mitra D., Gupta Y., 2008, MNRAS, 388, 261

Johnston S., Kramer M., Lorimer D. R., Lyne A. G., McLaughlin M., Klein B., Manchester R. N., 2006, MNRAS, 373, L6

Karastergiou A., Chennamangalam J., Armour W., Williams C., Mort B., Dulwich F., Salvini S., Magro A., et al. 2015, MNRAS, 452, 1254

Kargaltsev O., Pavlov G. G., Garmire G. P., 2007, ApJ, 660, 1413

Katz J. I., 2016, ArXiv e-prints 1604.01799

Keane E. F., Johnston S., Bhandari S., Barr E., Bhat N. D. R., Burgay M., Caleb M., Flynn C., et el. 2016, Nature 
Kern J. S., 2000, in Kramer M., Wex N., Wielebinski R., eds, IAU Colloq. 177: Pulsar Astronomy - 2000 and Beyond Vol. 202 of Astronomical Society of the Pacific Conference Series, Single Pulse Analysis of the Core-Dominated Pulsar B0611+22. p. 175

Kijak J., Dembska M., Lewandowski W., Melikidze G., Sendyk M., 2011, MNRAS, 418, L114

Kijak J., Gupta Y., Krzeszowski K., 2007, A\&A, 462, 699

Kijak J., Lewandowski W., Maron O., Gupta Y., Jessner A., 2011, A\&A, 531, A16

Kijak J., Tarczewski L., Lewandowski W., Melikidze G., 2013, ApJ, 772, 29

Kocz J., Bailes M., Barnes D., Burke-Spolaor S., Levin L., 2012, MNRAS, 420, 271

Kohler S., 2016, AAS Nova Highlights, p. 1086

Kondratiev V. I., Verbiest J. P. W., Hessels J. W. T., Bilous A. V., Stappers B. W., Kramer M., Keane E. F., Noutsos A., Osłowski S. e. a., 2015, ArXiv e-prints

Koo B.-C., Moon D.-S., Lee H.-G., Lee J.-J., Matthews K., 2007, ApJ, 657, 308

Kramer M., Karastergiou A., Gupta Y., Johnston S., Bhat N. D. R., Lyne A. G., 2003, A\&A, 407, 655

Kramer M., Lange C., Lorimer D. R., Backer D. C., Xilouris K. M., Jessner A., Wielebinski R., 1999, ApJ, 526, 957 
Kramer M., Lyne A. G., O’Brien J. T., Jordan C. A., Lorimer D. R., 2006, Science, 312,549

Kramer M., Xilouris K. M., Lorimer D. R., Doroshenko O., Jessner A., Wielebinski R., Wolszczan A., Camilo F., 1998, ApJ, 501, 270

Krishnakumar M. A., Mitra D., Naidu A., Joshi B. C., Manoharan P. K., 2015, ApJ, 804, 23

Kulkarni S. R., Ofek E. O., Neill J. D., 2015, ArXiv e-prints 1511.09137

Kulkarni S. R., Ofek E. O., Neill J. D., Zheng Z., Juric M., 2014, ApJ, 797, 70

Kuniyoshi M., Verbiest J. P. W., Lee K. J., Adebahr B., Kramer M., Noutsos A., 2015, MNRAS, 453, 828

Law C. J., Yusef-Zadeh F., Cotton W. D., Maddalena R. J., 2008, ApJS, 177, 255

Lawson K. D., Mayer C. J., Osborne J. L., Parkinson M. L., 1987, MNRAS, 225, 307

Lazio T. J. W., Cordes J. M., 1998, ApJ, 505, 715

Lehner L., Palenzuela C., Liebling S. L., Thompson C., Hanna C., 2012, Phys. Rev. D, 86, 104035

Lewandowski W., Kijak J., Gupta Y., Krzeszowski K., 2011, A\&A, 534, A66

Lewandowski W., Kowalińska M., Kijak J., 2015, MNRAS, 449, 1570

Lewandowski W., Rożko K., Kijak J., Melikidze G. I., 2015, ApJ, 808, 18 
Lorimer D. R., Bailes M., McLaughlin M. A., Narkevic D. J., Crawford F., 2007, Science, 318, 777

Lorimer D. R., Esposito P., Manchester R. N., Possenti A., Lyne A. G., McLaughlin M. A., Kramer M., Hobbs G., Stairs I. H., Burgay M., Eatough R. P., Keith M. J., Faulkner A. J., D’Amico N., Camilo F., Corongiu A., Crawford F., 2015, MNRAS, 450, 2185

Lorimer D. R., Faulkner A. J., Lyne A. G., Manchester R. N., Kramer M., McLaughlin M. A., Hobbs G., Possenti A., Stairs I. H., Camilo F., Burgay M., D’Amico N., Corongiu A., Crawford F., 2006, MNRAS, 372, 777

Lorimer D. R., Karastergiou A., McLaughlin M. A., Johnston S., 2013, MNRAS, 436, L5

Lorimer D. R., Kramer M., 2004, Handbook of Pulsar Astronomy. Cambridge University Press

Lorimer D. R., Kramer M., 2005, Handbook of Pulsar Astronomy. Cambridge University Press

Lorimer D. R., Kramer M., 2012, Handbook of Pulsar Astronomy. Cambridge University Press

Lorimer D. R., Lyne A. G., McLaughlin M. A., Kramer M., Pavlov G. G., Chang C., 2012, ApJ, 758, 141

Lorimer D. R., Yates J. A., Lyne A. G., Gould D. M., 1995, MNRAS, 273, 411 
Lyne A., Hobbs G., Kramer M., Stairs I., Stappers B., 2010, Science, 329, 408

Lyne A. G., Manchester R. N., 1988, Mon. Not. R. Astron. Soc., 234, 477

Lyne A. G., Manchester R. N., 1988, MNRAS, 234, 477

Lyne A. G., Stappers B. W., Freire P. C. C., Hessels J. W. T., Kaspi V. M., Allen B., Bogdanov S., Brazier A., et al. 2017, ApJ, 834, 72

Lyubarsky Y., 2014, MNRAS, 442, L9

Lyutikov M., Burzawa L., Popov S. B., 2016, ArXiv e-prints 1603.02891

Macquart J.-P., Kanekar N., 2015, ApJ, 805, 172

Macquart J.-P., Kanekar N., Frail D. A., Ransom S. M., 2010, ApJ, 715, 939

Macquart J.-P., Koay J. Y., 2013, ApJ, 776, 125

Madau P., Dickinson M., 2014, ARA\&A, 52, 415

Madsen G. J., Reynolds R. J., Haffner L. M., 2006, ApJ, 652, 401

Manchester R. N., Kramer M., Stairs I. H., Burgay M., Camilo F., Hobbs G. B., Lorimer D. R., Lyne A. G., McLaughlin M. A., McPhee C. A., Possenti A., Reynolds J. E., van Straten W., 2010, apj, 710, 1694

Manchester R. N., Lyne A. G., 1977, MNRAS, 181, 761

Manchester R. N., Lyne A. G., Camilo F., Bell J. F., Kaspi V. M., D’Amico N., McKay N. P. F., Crawford F., et al. 2001, MNRAS, 328, 17 
Marcote B., Paragi Z., Hessels J. W. T., Keimpema A., van Langevelde H. J., Huang Y., Bassa C. G., Bogdanov S., et al. 2017, ApJL, 834, L8

Maron O., Kijak J., Kramer M., Wielebinski R., 2000, A\&A, 147, 195

Maron O., Serylak M., Kijak J., Krzeszowski K., Mitra D., Jessner A., 2013, A\&A, $555, \mathrm{~A} 28$

Masui K., Lin H.-H., Sievers J., Anderson C. J., Chang T.-C., Chen X., Ganguly A., et al. 2015, Nature, 528, 523

McLaughlin M. A., Rea N., Gaensler B. M., Chatterjee S., Camilo F., Kramer M., Lorimer D. R., Lyne A. G., Israel G. L., Possenti A., 2007, ApJ, 670, 1307

Melrose D. B., 1995, Journal of Astrophysics and Astronomy, 16, 137

Melrose D. B., Yuen R., 2014, MNRAS, 437, 262

Mereghetti S., Pons J. A., Melatos A., 2015, Space Science Review, 191, 315

Mezger P. G., Henderson A. P., 1967, ApJ, 147, 471

Michel F. C., 1991, Theory of Neutron Star Magnetospheres. University of Chicago Press, Chicago

Mori K., Gotthelf E. V., Zhang S., An H., Baganoff F. K., Barrière N. M., Beloborodov A. M., Boggs S. E., Christensen F. E. e. a., 2013, ApJL, 770, L23

Muñoz J. B., Kovetz E. D., Dai L., Kamionkowski M., 2016, Physical Review Letters, 117,091301 
Newburgh L. B., Addison G. E., Amiri M., Bandura K., Bond J. R., Connor L., Cliche J.-F., Davis G., Deng M., et al. 2014, in Ground-based and Airborne Telescopes V Vol. 9145 of SPIE, Calibrating CHIME: a new radio interferometer to probe dark energy. p. $91454 \mathrm{~V}$

Newburgh L. B., Bandura K., Bucher M. A., Chang T.-C., Chiang H. C., Cliche J. F., Dave R., Dobbs M., et al. 2016, ArXiv e-prints 1607.02059

Nowakowski L. A., 1992, in Hankins T. H., Rankin J. M., Gil J. A., eds, IAU Colloq. 128: Magnetospheric Structure and Emission Mechanics of Radio Pulsars Four Pulsars with New Faces. p. 280

O’Dea C. P., 1998, PASP, 110, 493

Olbert C. M., Clearfield C. R., Williams N. E., Keohane J. W., Frail D. A., 2001, ApJL, 554, L205

Oster L., 1961, Reviews of Modern Physics, 33, 525

Pavlov G. G., Kargaltsev O., Wong J. A., Garmire G. P., 2009, ApJ, 691, 458

Pedlar A., Anantharamaiah K. R., Ekers R. D., Goss W. M., van Gorkom J. H., Schwarz U. J., Zhao J.-H., 1989, ApJ, 342, 769

Petroff E., Bailes M., Barr E. D., Barsdell B. R., Bhat N. D. R., Bian F., BurkeSpolaor S., Caleb M., et al. 2015, MNRAS, 447, 246

Petroff E., Keane E. F., Barr E. D., Reynolds J. E., Sarkissian J., Edwards P. G., 
Stevens J., Brem C., Jameson A., Burke-Spolaor S., Johnston S., Bhat N. D. R., Kudale P. C. S., Bhandari S., 2015, MNRAS, 451, 3933

Pilkington J. D. H., Hewish A., Bell S. J., Cole T. W., 1968, Nature, 218, 126

Piro A. L., 2016, ArXiv e-prints 1604.04909

Press W. H., Teukolsky S. A., Vetterling W. T., Flannery B. P., 2002, Numerical recipes in $\mathrm{C}++$ : the art of scientific computing. Cambridge University Press

Rajwade K., Lorimer D. R., Anderson L. D., 2016a, MNRAS, 455, 493

Rajwade K., Lorimer D. R., Anderson L. D., 2016b, MNRAS, 455, 493

Rankin J. M., 1983, ApJ, 274, 333

Rankin J. M., 1983a, Astrophys. J., 274, 333

Rankin J. M., 1983b, Astrophys. J., 274, 359

Rankin J. M., 1986, ApJ, 301, 901

Rankin J. M., 1990, ApJ, 352, 247

Rankin J. M., 1990, Astrophys. J., 352, 247

Rankin J. M., 1993, ApJS, 85, 145

Rankin J. M., 1993, Astrophys. J., 405, 285

Rankin J. M., Ramachandran R., 2003, ApJ, 590, 411

Rankin J. M., Wright G. A. E., Brown A. M., 2013, MNRAS, 433, 445 
Ransom S. M., Demorest P., Ford J., McCullough R., Ray J., DuPlain R., Brandt P., 2009, in American Astronomical Society Meeting Abstracts \#214 Vol. 214 of American Astronomical Society Meeting Abstracts, GUPPI: Green Bank Ultimate Pulsar Processing Instrument. p. 605.08

Ravi V., Shannon R. M., Bailes M., Bannister K., Bhandari S., Bhat N. D. R., Burke-Spolaor S., Caleb M., Flynn C., Jameson A., Johnston S., Keane E. F., Kerr M., Tiburzi C., Tuntsov A. V., Vedantham H. K., 2016, Science, 354, 1249

Rickett B. J., 1977, ARA\&A, 15, 479

Romani R. W., Yadigaroglu I.-A., 1995, ApJ, 438, 314

Rowlinson A., Bell M. E., Murphy T., Trott C. M., Hurley-Walker N., Johnston S., Tingay S. J., Kaplan D. L., et al. 2016, MNRAS, 458, 3506

Ruderman M. A., Sutherland P. G., 1975, Astrophys. J., 196, 51

Rybicki G. B., Lightman A. P., 1979, Radiative processes in astrophysics

Sankrit R., Hester J. J., Scowen P. A., Ballester G. E., Burrows C. J., Clarke J. T., Crisp D., Evans R. W., et al. 1998, ApJ, 504, 344

Scheuer P. A. G., 1968, Nature, 218, 920

Schödel R., Eckart A., Alexander T., Merritt D., Genzel R., Sternberg A., Meyer L., Kul F., Moultaka J., Ott T., Straubmeier C., 2007, A\&A, 469, 125

Seymour A. D., Lorimer D. R., Ridley J. P., 2014, MNRAS, 439, 3951 
Sieber W., 1973, A\& A, 28, 237

Sieber W., 1973, Astron. Astrophys., 28, 237

Spitler L. G., Cordes J. M., Hessels J. W. T., Lorimer D. R., McLaughlin M. A., Chatterjee S., Crawford F., Deneva J. S., et al. 2014, ApJ, 790, 101

Spitler L. G., Scholz P., Hessels J. W. T., Bogdanov S., Brazier A., Camilo F., Chatterjee S., Cordes J. M., et al. 2016, Nature, 531, 202

Staveley-Smith L., Wilson W. E., Bird T. S., Disney M. J., Ekers R. D., Freeman K. C., Haynes R. F., Sinclair M. W., Vaile R. A., Webster R. L., Wright A. E., 1996, PASA, 13, 243

Stovall K., Lynch R. S., Ransom S. M., Archibald A. M., Banaszak S., Biwer C. M., Boyles J., Dartez L. P., et al. 2014, ApJ, 791, 67

Strüder L., Briel U., Dennerl K., Hartmann R., Kendziorra E., Meidinger N., Pfeffermann E., Reppin C. e. a., 2001, A\&A, 365, L18

Sturrock P. A., 1971, ApJ, 164, 529

Suleymanova S. A., Izvekova V. A., Rankin J. M., Rathnasree N., 1998, Journal of Astrophysics and Astronomy, 19, 1

Sutton J. M., 1971, MNRAS, 155, 51

Tauris T. M., Langer N., Kramer M., 2012, MNRAS, 425, 1601 
Tendulkar S. P., Bassa C. G., Cordes J. M., Bower G. C., Law C. J., Chatterjee S., Adams E. A. K., Bogdanov S., et al. 2017, ApJL, 834, L7

Thompson C., Duncan R. C., 1993, Astrophys. J., 408, 194

Thornton D., Stappers B., Bailes M., Barsdell B., Bates S., Bhat N. D. R., Burgay M., Burke-Spolaor S., et al. 2013, Science, 341, 53

Timokhin A. N., 2010, MNRAS, 408, L41

Tingay S. J., Trott C. M., Wayth R. B., Bernardi G., Bowman J. D., Briggs F., Cappallo R. J., Deshpande A. A., et al. 2015, AJ, 150, 199

Truemper J., Pietsch W., Reppin C., Voges W., Staubert R., Kendziorra E., 1978, ApJL, 219, L105

Turner M. J. L., Abbey A., Arnaud M., Balasini M., Barbera M., Belsole E., Bennie P. J., Bernard J. P. e. a., 2001, A\& A, 365, L27

van Straten W., Demorest P., Khoo J., Keith M., Hotan A., et al., 2011, PSRCHIVE: Development Library for the Analysis of Pulsar Astronomical Data, Astrophysics Source Code Library

Vedantham H. K., Ravi V., Hallinan G., Shannon R., 2016, ArXiv eprints: 1606.06795

Vink J., Bamba A., Yamazaki R., 2011, ApJ, 727, 131

Wang N., Manchester R. N., Johnston S., 2007, MNRAS, 377, 1383 
Welsh B. Y., Sallmen S., 2003, A\& A, 408, 545

Weltevrede P., Edwards R. T., Stappers B. W., 2006, A\&A, 445, 243

Weltevrede P., Johnston S., 2008, MNRAS, 391, 1210

Wharton R. S., Chatterjee S., Cordes J. M., Deneva J. S., Lazio T. J. W., 2012, ApJ, 753, 108

Wijnholds S. J., van Cappellen W. A., 2011, IEEE Transactions on Antennas and Propagation, 59, 1981

Woods P. M., Thompson C., 2006, Soft gamma repeaters and anomalous X-ray pulsars: magnetar candidates. pp 547-586

Wright G. A. E., Fowler L. A., 1981, A\&A, 101, 356

Yamasaki S., Totani T., Kawanaka N., 2016, MNRAS, 460, 2875

Yao J. M., Manchester R. N., Wang N., 2017, ApJ, 835, 29

Zackay B., Ofek E. O., 2014, ArXiv e-prints 1411.5373

Zhou B., Li X., Wang T., Fan Y.-Z., Wei D.-M., 2014, Phys. Rev. D, 89, 107303 